\title{
LSM2-8 and XRN-2 contribute to the silencing of H3K27me3-marked genes through targeted RNA decay
}

Anna Mattout", Dimos Gaidatzis, Jan Padeken, Christoph Schmid, Florian Aeschlimann ${ }^{+}$, Véronique Kalck and Susan M. Gasser ${ }^{*+}$

Friedrich Miescher Institute for Biomedical Research, Maulbeerstrasse 66, CH-4058 Basel, Switzerland

\#current address: Université Paul Sabatier- CNRS UMR 5088, 118 Route de Narbonne, 31062 Toulouse, France

+University of Basel, Faculty of Science, Klingelbergstrasse 70, CH-4056 Basel, Switzerland

* Correspondence to: susan.gasser@fmi.ch

Running title: Polycomb silencing and RNA degradation

Keywords: LSM8, XRN2, RNA degradation, Polycomb, PRC2, mes-2, C. elegans, Heterochromatin silencing, H3K27me3, epigenetics, LSM complex. 


\section{Summary}

In fission yeast and plants, RNA-processing pathways contribute to constitutive and facultative heterochromatin silencing, complementing well-characterized pathways of transcriptional repression. However, it was unclear whether this additional level of regulation occurs in metazoans. Here we describe a pathway of silencing in C. elegans somatic cells, in which the highly conserved, RNA binding complex LSM2-8 selectively silences heterochromatic reporters and endogenous genes bearing the Polycomb mark H3K27me3. Importantly, the LSM2-8 complex works cooperatively with XRN-2, a 5'-3' exoribonuclease, and disruption of the pathway leads to mRNA stabilization. This selective LSM2-8-mediated RNA degradation does not target nor depend on $\mathrm{H} 3 \mathrm{~K} 9 \mathrm{me} 2 / \mathrm{me} 3$, unlike previously described pathways of heterochromatic RNA degradation. Intriguingly, the loss of LSM2-8 coincides with a localized drop in H3K27me3 levels on $l s m-8$-sensitive loci only. Together this defines a mechanism of RNA degradation that selectively targets a subset of H3K27me3-marked genes, revealing an unrecognized layer of regulation for facultative heterochromatin in animals. 


\section{Introduction}

The organization of DNA sequences into highly condensed, dark-staining heterochromatin correlates with reduced gene expression (Saksouk et al., 2015; Trojer and Reinberg, 2007; Wenzel et al., 2011). Heterochromatin can be classified as constitutive or facultative heterochromatin. $\mathrm{H} 3 \mathrm{~K} 9 \mathrm{me} 3$ is the histone modification that characterizes constitutive heterochromatin, which is found most often on non-coding raepetitive elements (Saksouk et al., 2015; Zeller et al., 2016), while Polycomb-mediated trimethylation of H3K27 is the hallmark of facultative heterochromatin. H3K27me3 defines a repressive state that silences genes as a function of temporal and spatial conditions, for instance, during development (Gaydos et al., 2014; Trojer and Reinberg, 2007). Whereas transcriptional repression is believed to be the primary level of regulation through which both constitutive and facultative heterochromatin act, robust pathways that silence at co- and posttranscriptional levels have been documented in fission yeast and plants (Buhler, 2009; Wang et al., 2016).

Over a decade ago it was shown that transcription and noncoding RNAs were involved in the establishment of heterochromatin-mediated repression in fission yeast (Grewal and Elgin, 2007; Moazed, 2009). Counterintuitively, ncRNA transcripts were shown to promote RNAi-mediated assembly of centromeric heterochromatin by providing both small RNAs and a scaffold to recruit chromatin-modifying enzymes through the RITS complex (Buhler, 2009; Noma et al., 2004; Wang et al., 2016). Additionally, RNAi-independent RNA degradation mechanisms that use the exosome were implicated in constitutive heterochromatic gene silencing in S. pombe (Buhler et al., 2007). The exosome was also implicated in heterochromatic repeat silencing in Drosophila (Eberle et al., 2015), and at centromeric and pericentromeric loci in Arabidopsis (Shin et al., 2013). RNA degradation was also suggested to contribute to rDNA stability and subtelomeric silencing in budding yeast (Vasiljeva et al., 2008).

In $S$. pombe, a variety of RNA associated factors were shown to promote $\mathrm{H} 3 \mathrm{~K} 9 \mathrm{me} 2 / 3$ silencing in a partially redundant manner, acting through mechanisms that process RNA transcripts. These include HP1(Swi6) (Keller et al., 2012), Red1 and Mmi1 (Egan et al., 2014; Touat-Todeschini et al., 2017; Yamanaka et al., 2013; Zofall et al., 2012), Pla1 (Yamanaka et al., 2013), Pab2 (StAndre et al., 2010; Yamanaka et al., 2013), and Dhp1/Xrn2 (Chalamcharla et al., 2015; Tucker et al., 2016). These studies document multiple links between RNA factors and constitutive 
heterochromatin in plants and yeast, yet no compelling parallel has been reported to date for facultative heterochromatin in animals. Using a genome-wide derepression screen, we have uncovered and characterized such a pathway in embryos and differentiated tissues of C. elegans.

In an earlier genome-wide RNAi screen that monitored the derepression of an integrated heterochromatic reporter in $C$. elegans, we identified 29 factors that were essential for heterochromatin silencing in embryos (Towbin et al., 2012; Fig. 1A,B). While most of the validated hits were chromatin modifiers and transcription-related proteins (Towbin et al., 2012), among them were three subunits of the RNA-binding Like-SM (LSM) complexes (gut-2/lsm-2, $l s m-5$ and $l s m-6)$. LSM proteins are highly conserved throughout evolution, with the C. elegans proteins sharing up to $94 \%$ homology with their human counterparts (Fig. S1A). The two LSM complexes, LSM1-7 (cytoplasmic) and LSM2-8 (nuclear), were shown to function in RNA metabolism and splicing, but not in transcription per se (Beggs, 2005; Cornes et al., 2015; Golisz et al., 2013; Kufel et al., 2004; Perea-Resa et al., 2012; Tharun, 2009). The cytoplasmic LSM1-7 complex partners with decapping enzymes, which render RNA sensitive to the $5^{\prime}$ to $3^{\prime} \mathrm{XRN}-1$ exonuclease activity, while the LSM2-8 complex has been proposed to work with XRN-2 to promote nuclear RNA decay (Beggs, 2005; Tharun, 2009). No role for LSM proteins in heterochromatin silencing has been previously reported.

Our initial screen implicated LSM-2, LSM-5 and LSM-6 in gene silencing based on robust GFP derepression of a heterochromatic reporter in worm embryos, after RNAi-mediated knockdown of the individual genes (Fig. 1A,B). In this de-silencing assay, the heterochromatic reporters consisted of an integrated array of several hundred copies of a plasmid, carrying a GFP-encoding reporter gene driven by a ubiquitously expressed promoter that was repressed in wild-type $C$. elegans. In a copy-number dependent manner, these heterochromatic reporters acquire the histone modifications $\mathrm{H} 3 \mathrm{~K} 9 \mathrm{me} 2 / 3$ and $\mathrm{H} 3 \mathrm{~K} 27 \mathrm{me} 3$ and are sequestered at the nuclear envelope, thereby mimicking endogenous heterochromatin (Meister et al., 2010; Towbin et al., 2010; Wenzel et al., 2011). Loss of either of the two H3K9 methyltransferases (MET-2, SET-25) or the EZH2 homolog, MES-2, which methylates H3K27, led to reporter derepression (Towbin et al., 2012).

Here we examined in depth the pathway by which LSM proteins contribute to heterochromatic silencing in $C$. elegans. We find that the LSM2-8 complex works with XRN-2 in a posttranscriptional RNA decay pathway that selectively targets transcripts arising from endogenous 
genes enriched for H3K27me3. The LSM1-7 complex is not involved in this silencing pathway. LSM8-mediated silencing can occur at all developmental stages, and in all somatic cells. Not only are the LSM8-sensitive loci preferentially enriched for Polycomb-mediated H3K27me3, but the level of H3K27me3 on these genes drops in animals lacking $l s m-8$. This argues for a feedback loop, in which LSM2-8 serves as an intermediary that triggers the degradation of specific transcripts while concomitantly enhancing a repressive chromatin state. Upon the loss of $l s m-8$, the animals are sterile, have premature death phenotypes and misexpress the HOX gene egl-5. This is the first molecular pathway of RNA degradation in any organism that is correlated with the characteristic histone modification of facultative heterochromatin, H3K27me3. It shows that the selective degradation of heterochromatic transcripts in the nucleus can supplement repression on the level of transcription to silence target genes in animals.

\section{Results}

\section{LSM proteins selectively silence heterochromatic reporters throughout somatic differentiation}

The initial genome-wide RNAi screen for factors involved in heterochromatin silencing monitored let-858p::gfp derepression from the heterochromatic reporter pkIs1582 (strain GW306), in embryos, (Fig. 1A,B; Table S1; Towbin et al., 2012). We used RNAi against $l s m-2$, $l s m-5$ and $l s m$ 6 to analyze the derepression/ increased expression of GFP throughout C. elegans development. We found a reproducible and robust derepression at all stages i.e. in embryos, L1 to L4 larval stages and in adult worms (Fig. 1C, Fig. S1B). Moreover, as shown by fluorescence microscopy, elevated levels of GFP were found in nearly every somatic cell type (Fig. 1C, Fig. S1B).

The ubiquitous derepression of the heterochromatic reporter allowed us to quantify GFP expression following RNAi using the COPAS Biosorter. The Biosorter uses flow cytometry to quantify fluorescence of single worms with high throughput, generating robust population-wide measurements. We find highly significant upregulation of GFP in populations of L1 larvae following RNAi for $l s m-2, l s m-5$ and $l s m-6$, as compared to the control/mock RNAi treated worms (L4440; Fig. S1C). The differences in derepression between $l s m-2, l s m-5$ and $l s m-6$ most likely reflect the different knock-down efficiencies of the respective RNAi clones (Fig. S1C). The 
population-wide effect of $l s m-2$ RNAi was as strong as RNAi against the HMT MES-4, a positive control that ablates H3K36 methylation (Towbin et al., 2012).

Given the various RNA processing functions attributed to LSM proteins (Tharun, 2009), we asked whether the derepression we scored upon $l s m$ depletion acted uniquely on the heterochromatic reporter used. We tested $l s m$ RNAi on the expression of four independent heterochromatic reporters, each with a distinct combination of promoter, reporter gene (encoding GFP or mCherry), 3' UTR, and site of integration (Fig. 1D, Table S1). We also tested for effects of $l s m-2$, $l s m-5$ or lsm-6 RNAi on two euchromatic reporters respectively from the strains GW849 and GW1108, which carry single copy transgenes (encoding GFP or mCherry) integrated into nonheterochromatic regions of the genome. Whereas all the heterochromatic reporters tested showed significant derepression (Fig. 1D,E, Fig. S1C), the euchromatic reporters showed no change in expression following lsm RNAi (Fig. 1D,E, Fig. S1F-G). This specificity was confirmed not only for RNAi against $l s m-2,-5$ and -6 , but also for $l s m-7$ RNAi (Fig. S1B,D), another subunit of the LSM complexes.

The lack of response observed for the euchromatic reporters does not reflect differences in the basal or background fluorescence level, given that the four heterochromatic reporters, despite having different initial GFP expression levels, were all derepressed following $l_{s m-7}$ RNAi (Fig. S1D). Moreover, neither $l s m-6$ nor $l s m-7$ RNAi had an effect on a highly expressed single copy transgene (eft-3p::gfp, GW1108), whose GFP signal is higher than the repressed, let-858p::gfp heterochromatic reporter (from the strain GW306; Fig. 1E), nor on the euchromatic cec4p::mCherry reporter in GW849, which is expressed at a lower level than this heterochromatic reporter (Fig. S1F,G).

Finally, to make sure that the increased expression reflects changes in mRNA and not altered protein synthesis or turnover, we monitored the relative increase in $g f p$ mRNA levels in GW306 and GW1108 by qPCR. Indeed, the heterochromatic let-858p::gfp reporter showed higher steadystate levels of mRNA following $l s m-6$ and $l s m-7$ RNAi, while the euchromatic eft-3p::gfp mRNA did not (Fig. 1F). Taken together, this suggests that the C. elegans LSM factors regulate mRNA levels, rather than protein turnover or translation, to silence specifically reporters with heterochromatic features. This occurs both during somatic cell differentiation and in post-mitotic cells throughout development. 


\section{RNAi implicates LSM2-7 and XRN-2, but not LSM-1 and XRN-1, in reporter repression}

The LSM proteins 2 through 7 are shared subunits of two related complexes: the LSM1-7 complex is primarily cytoplasmic, while the LSM2-8 complex is nuclear (Beggs, 2005; Fig. 1G). The two complexes also differ in their co-factors. The cytoplasmic LSM1-7 complex acts together with the 5 ' $\rightarrow$ 3'exoribonucleases, XRN-1 and the decapping enzymes DCAP-1 and DCAP-2 to mediate cytoplasmic RNA decay (Tharun, 2009). The LSM2-8 complex on the other hand was suggested to work in concert with the nuclear $5^{\prime} \rightarrow 3^{\prime}$ 'exoribonucleases XRN-2 (Tharun, 2009). To determine which of the two LSM complexes contribute to heterochromatic gene silencing, we compared reporter derepression after RNAi against $l s m-1$, which is unique to the LSM1-7 complex, with the levels after RNAi against two shared subunits, $l s m-4$ and $l s m-7$. Strong heterochromatic reporter derepression was scored upon knockdown of $l s m-4$ and $l s m-7$, while no effect was observed following RNAi against $l s m-1$ (Fig. $1 \mathrm{H})$. RNAi efficiency was similar in $l s m-1$ and $l s m-7$ RNAi treated larvae (Fig. S1E). In addition, RNAi against the LSM1-7 associated factors, dcap-2 and $x r n-1$, failed to provoke heterochromatic reporter derepression, while RNAi against $x r n$-2 did (Fig. 1H). In summary, our RNAi studies argued that knockdown of the related LSM proteins, LSM-2, $-4,-5,-6$, and -7 , but not of LSM-1 or its associated factors, triggered heterochromatin reporter derepression.

\section{Deletion of $l s m-8$ leads to efficient derepression, while $l s m-1$ or dacp- 2 deletions do not}

The failure of $l s m-1$ RNAi to derepress the reporter suggested that the LSM1-7 complex is not involved in heterochromatic silencing, and suggested that the LSM2-8 complex is. Because attempts to use RNAi to downregulate $l s m-8$, the only unique subunit of LSM2-8, were ineffective in our hands, we generated a full $l s m-8$ deletion by CRISPR/Cas9, replacing the $l s m-8$ locus with a red fluorescent marker gene with pharynx-specific expression. This allows tracking of the null allele (Fig. 2A). We found that somatic development the homozygous $l \mathrm{sm}-8^{-/-}$worms - including gonad formation - was similar to wild-type worms up through the L3 and L4 larval stages (Fig. $\mathrm{S} 2 \mathrm{~A}, \mathrm{~B}$ ), yet the adult homozygous mutant was sterile. At the young adult stage (after L4) the gonads in $l \mathrm{sm}-8^{-/}$animals became abnormal and failed to support oocyte maturation (Fig. S2C). To stably maintain the $l s m-8$ deletion we balanced the deletion with the $n T 1$ [qIs 51$]$ balancer, which expresses a GFP marker in the pharynx. This allowed us to sort $l s m-8^{-/-}$homozygous from heterozygous $l s m-8^{+/}$worms for further analysis because heterozygous $l s m-8^{-/+}$worms would 
have both red and green fluorescence in the pharynx, and homozygous $l s m 8^{-/-}$worms express only red (Fig. 2B).

Although the $l s m-8^{-/-}$animals developed to adulthood, they had protruding vulva, showed empty cavities or vacuoles in differentiated tissues, died prematurely and were 100\% sterile (no oocytes; Fig. 2B, Fig. S2C). The presence of cavities coincides mainly with premature death in adult stages (Fig. 2B). Unlike $l s m-1$ mutants (Cornes et al., 2015), worms lacking $l s m-2$ or $l s m-5$, are phenotypically similar to $l s m-8^{-/-}$mutants, with protruding vulva, abundant vacuoles and $100 \%$ sterility (Fig. S2C).

Using pharyngeal fluorescence to distinguish homozygous $l s m 8^{-/-}$from heterozygous $l s m 8^{+/-}$ animals in the F1 progeny, we monitored the repression of the heterochromatic reporter pkIs 1582 integrated in the strain carrying the $l s m-8$ null allele. Derepression was as strong as with $l s m-7$ RNAi and was restricted to homozygous $l s m-8^{-/-}$animals (red pharynx; Fig. 2D). To be certain that the phenotype was specific for the LSM2-8 complex, we obtained and backcrossed homozygous $l s m-1^{-/}$and $d$ cap $-2^{-/}$animals, coupling these genomic deletions with the same heterochromatic reporter. While the $l s m-8^{-/}$larvae had uniformly GFP-positive nuclei due to reporter derepression throughout the animal, none of the $l s m-1$ and $d$ cap-2 null animals had GFP signals above the wildtype (WT) background levels (Fig. 2E). We conclude that the loss of heterochromatic silencing stems from loss of a functional LSM2-8 complex, while there is no indication that the LSM17/DCAP-2 pathway of cytoplasmic RNA degradation by XRN-1 is involved.

Given the sterility and severe germline phenotype that was manifest in the $l s m-8^{-/-}$animals, we next examined derepression of the reporter within the gonad. Whereas the heterochromatic reporter pkIs 1582 was derepressed in the somatic gonadal cells (distal tip cell, gonadal sheath, and spermathecal cells), as well as in nearly all somatic cells in L4 larvae both after $l s m-7 R N A i$ and in $l s m-8^{-/-}$worms, the germline itself (germ cells, in the dashed red line) had no GFP expression (Fig. S2D-F). We wondered if this might reflect redundancy with the piRNA pathway, which mediates germline specific silencing (Shirayama et al., 2012). However, there was again no derepression in the $l s m 8^{-/-}$germ cells (GW1119) when the mutation was coupled with RNAi against the piRNArelated factor, csr-1 (Fig. S2G), or prg-1 (data not shown). Thus LSM2-8 pathway seems to affect primarily reporter expression in somatic cells.

\section{LSM2-8 is required to maintain silent endogenous heterochromatin}


To examine changes in endogenous transcript levels provoked by loss of LSM-8, we performed a strand-specific total RNA-seq on WT and homozygous $l s m-8^{-/-}$sorted L3 larvae (Fig. 3A). In order to compare the LSM2-8 silencing pathway with the well-characterized pattern of repression mediated by H3K9 methylation (Towbin et al., 2012; Zeller et al., 2016), transcriptome data from sorted $l s m-8^{-/}$L3 larvae was compared with data obtained in parallel from larvae carrying the double deletion $m e t-2^{-/-}$set-25/- (which lack all detectable H3K9me), or the triple mutation, met$2^{-/-}$set- $25^{--} ; l s m-8^{-/}$. In each case, the WT, single, double and triple mutant worms were sorted by fluorescence (no pharyngeal green signal indicating $l s m 8^{-/-}$) and by size, to generate uniform populations of L3 stage larvae for each genotype (Fig. 3A). Slight shifts in timing between samples can occur even among larvae at the same developmental stage due to the time required for the sorting process. Therefore, we chose and matched pairwise the samples from the four genotypes that were the closest in timing. We scored for developmental timing using a characteristic temporal fluctuation of a subset of somatic genes that were shown to be robust markers for developmental synchrony (Hendriks et al., 2014) (Fig. S3A).

Deletion of $l s m-8$ led to the up-regulation of transcripts of 122 genes (false discovery rate (FDR) $<0.05$ and fold change $(\mathrm{Fc})>4$ ), while only 9 genes were down-regulated (Fig. 3B, Table S2). Using less stringent cut-off values $(\mathrm{Fc}>2)$, there were 1332 genes selectively upregulated in $l s m$ $8^{-/}$larvae. We confirmed that expression differences found between the mutant and WT worms (FDR $<0.05$ and $\mathrm{Fc}>4$ ) cannot be attributed to the slight differences in timing between samples, since the expression changes that stem from differences in developmental timing (rising genes, Fig. S3B) did not reflect the genes upregulated in $l s m-8^{-/}$L3 larvae or in the other mutants (Fig. 3B).

We looked for additivity or epistasis between the LSM2-8 and H3K9me repression pathways by comparing genome-wide RNA-seq data from the single, double and triple mutants. While the loss of H3K9 methylation (met-2 set-25) led to the strong upregulation of 219 genes (FDR $<0.05$ and Fc $>4$ ), only $36 \%$ of these overlapped with those derepressed in the $l s m-8^{-/}$mutant (Fig. S3C, yellow; Table S2). In addition, there are large subsets of derepressed genes that are upregulated exclusively in the $l s m-8$ or the met-2 set-25 mutant, arguing that the pathways are largely independent (Fig. S3C, blue and pink shading). Consistent with this hypothesis, the triple mutant

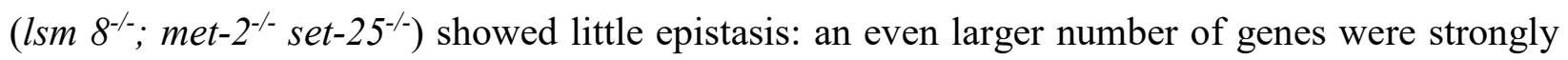


derepressed (FDR $<0.05$ and $\mathrm{Fc}>4$ ) in the triple mutant than in either $l s m-8$ or the met-2 set-25 strains (367 genes vs 122 and 219 respectively; Table S2, Fig. S3D). To illustrate this additivity, we selected a group of genes that were mildly upregulated upon loss of either $l s m-8$ or met-2 set25 ( $\mathrm{Fc}<2$; red boxed area in graph Fig. S3D) and examined their behavior in the triple mutant. The vast majority were derepressed $>4$-fold in the triple mutant (orange spots in Fig S3D, Table S2). Consistent with this, $g f p$ expression from the heterochromatic reporter, which bears both H3K9 and H3K27 methylation, also showed an additive relationship (Fig. S3E). We conclude that the LSM2-8 pathway of silencing for endogenous loci is distinct from that mediated by H3K9 methylation and does not depend on $\mathrm{H} 3 \mathrm{~K} 9 \mathrm{me} 3$, even though some genes were targeted by both pathways.

The additivity of phenotypes extends beyond gene expression. As indirect support for LSM2-8 and $\mathrm{H} 3 \mathrm{~K} 9 \mathrm{me}$ acting on parallel pathways, we note that unlike the $l s m-8$ deletion which strongly enhanced premature lethality during somatic growth over 10 days, the loss of H3K9 methylation alone does not (Fig. S3F), but rather slows development in a stochastic manner (Zeller et al., 2016). As expected, in worms bearing the combination of met-2 set-25 with the $l s m-8$ null allele, we observed enhanced lethality, i.e., the mutations were not epistatic, but additive (Fig. S3F).

\section{Over 93\% of LSM2-8 silenced genes bear H3K27 trimethylation}

Given that heterochromatic reporters, but not euchromatic reporters, are upregulated upon loss of lsm-8, we examined whether the genes upregulated by loss of LSM-8 share a common set of histone modifications. To do so, we plotted our L3 RNA-seq data against the normalized ChIPseq data for common histone modifications found the genome of WT L3 larvae, generated by ModEncode (Table S3, Fig. 3C-E, Fig. S4A). In worms, as in most organisms, H3K4me2, H3K4me3, and H3K27ac are associated with active genes (Ho et al., 2014; Liu et al., 2011; Wenzel et al., 2011), while H3K9me2/3 and H3K27me3 are repressive histone marks that co-localize with heterochromatin (Ahringer and Gasser, 2018; Wenzel et al., 2011). We found that the genes strongly derepressed in the $l s m-8^{-/-}$mutant $(\mathrm{Fc}>4$ and $\mathrm{FDR}<0.05)$, were depleted for the active marks, as well as for H3K9me1 in WT larvae (Fig. 3C-D; Fig. S4A). When lsm-8-upregulated genes were plotted against their relative enrichment for repressive marks, we found a striking correlation of LSM-8 sensitivity with the repressive Polycomb mark, H3K27me3. Over 95\% of the genes that were derepressed in the $l s m-8^{-/-}$mutant were enriched for H3K27me3 (Fig. 3D, Fig. 
$\mathrm{S} 4 \mathrm{~A})$. This was true not only for the genes that met our most stringent cut-off values ( $\mathrm{Fc}>4$ and FDR $<0.05$ ), but for the genes upregulated between 2 - and 4-fold by $l s m-8$ ablation (Fig. 3D, Tables S2-S5). In contrast, only $20 \%$ of the targeted genes were enriched for H3K9me2, a value that matches the genome-wide presence of H3K9me2-marked genes in L3 larvae, and roughly $40 \%$ of the upregulated genes carried H3K9me3 (Fig. 3D).

H3K27me3 and H3K9me3 ChIP-seq signals co-localize more frequently over the C. elegans genome, than they do in mammals or flies (Ho et al., 2014). We therefore examined the relationship of H3K27 and H3K9 methylation by examining to which extent the marks colocalize on LSM-8silenced genes. First, we note that in WT L3 larvae, 41\% of H3K27me3-marked genes genomewide, also bear H3K9me3 (ModEncode). We find a similar rate of H3K9me3 on LSM2-8 targets (40-43\%), among which 100\% are also H3K27me3 positive (Fig. 3D, Fig. S4A; Tables S2-S4). Thus, the presence of $\mathrm{H} 3 \mathrm{~K} 9 \mathrm{me} 3$ on LSM-8 target genes simply reflects the rate at which the two marks coincide genome-wide, while the enrichment of H3K27me3 on $l s m-8$ sensitive genes in L3 larvae $(95 \%)$ is highly significant $\left(\mathrm{p}<4.2 \mathrm{e}^{-24}\right)$. Taken together, we conclude that the histone mark that characterizes LSM2-8-regulated genes is H3K27me3. Consistent with its role in Polycombmediated repression (Conway et al., 2015; Grossniklaus and Paro, 2014; Liu et al., 2011; Margueron and Reinberg, 2011), we found that most of the genes significantly upregulated by the loss of LSM-8, are genes that have very low steady-state expression levels in WT worms (Fig. 3E, Fig. S4B).

In further analysis we asked whether the $l s m$ - 8 -sensitive genes had any specific distribution along C. elegans chromosomes. This is particularly relevant, given that constitutively repressed $\mathrm{H} 3 \mathrm{~K} 9 \mathrm{me} 2 / \mathrm{me} 3$ heterochromatin is enriched on chromosomal arms and depleted from chromosome cores (Ahringer and Gasser, 2018). In contrast to this, much like the distribution of the H3K27me3 mark itself (Ho et al., 2014), the genes sensitive to the loss of $l s m-8$ were found both in autosomal core regions and along chromosome arms (Fig. S4C). We asked whether other pathways of repression at the L3 larval stage show a similar preference for H3K27me3-marked genes. Performing a similar analysis of chromatin marks on cell cycle genes regulated by the Rblike repressor, LIN-35, and on developmental genes repressed by PRG-1, a PIWI protein, showed no bias toward H3K27me3 modification (prg-1 pathway) or even depletion for this mark for the lin-35 pathway (Fig. S4D). This reinforces our argument that the link of LSM2-8 to Polycomb- 
marked genes is specific. Interestingly, GO term analysis suggests that the genes silenced by LSM8 are enriched for genes expressed during the innate immune response, body morphogenesis and cell shape regulation (Table S5), processes that were found to be regulated by Polycomb in other species.

To assess whether LSM2-8 targets H3K27me3-marked genes in other developmental stages than L3 larvae, we performed total RNA-seq on synchronized and sorted WT and homozygous $l s m-8^{-/-}$ at the L1 larval stage. Deletion of $l s m-8$ led to the up-regulation of transcripts of 151 genes (FDR $<0.05$ and $\mathrm{Fc}>4$ ), while 59 genes were down-regulated (Fig. S4E, Tables S2, S4). Using Fc >2, 1501 genes were upregulated in $l s m-8^{-/}$. We find a significant $\left(\mathrm{p}<1.17 \mathrm{e}^{-12}\right)$ but small subsets of genes (22) that are upregulated upon LSM-8 ablation in both L1 and L3 larval stages and those genes seem to be involved in the immune response (Fig. S4F, Tables S2, S4). Importantly, the L1 stage genes regulated by LSM2-8 are significantly depleted for histone marks associated with euchromatin and are significantly $\left(\mathrm{p}<2.2 \mathrm{e}^{-25}\right)$ enriched for the Polycomb mark, H3K27me3 (Fig. S4G, Table S3). Notably, 93\% of the genes silenced by LSM2-8 in L1 larvae are found to carry the Polycomb mark.

\section{Cell-specific HOX gene up-regulation by $l s m-8$ ablation in specific cell types}

Across all multicellular species, the methylation histone H3K27 by the PRC2 complex leads to cell-type or stage-specific repression of genes implicated in development (Conway et al., 2015; Grossniklaus and Paro, 2014; Liu et al., 2011; Margueron and Reinberg, 2011; Patel et al., 2012), In C. elegans, PRC2 consists of MES-2/E(z)/EZH2, MES-3, and MES-6/Esc (Gaydos et al., 2014; Ketel et al., 2005; Yuzyuk et al., 2009). We asked whether the loss of LSM-8 reduced the expression of the Polycomb homologs, impairing catalysis of H3K27me3. However, RNA-seq data in $l s m-8$ deficient worms showed no drop in mes-2, mes-3 or mes- 6 expression, as well as no drop in the expression of sor-1 and sop-2 the PRC1-like factors (Table S2), ruling out this potential indirect mechanism.

Intriguingly, HOX genes, the canonical targets of H3K27me3, were not among the strongly upregulated genes in the $l s m-8^{-/-}$transcriptome. We reasoned that the transcripts of such genes might not be detected by RNA-seq of $l s m-8$ larvae, if their expression occurred only in a limited number of cells. Indeed, cell-type specific repression / derepression events are easily masked in 
RNA-seq datasets of whole animals. In C. elegans, the best conserved HOX cluster repressed by Polycomb includes lin-39 (essential for development of the vulva), ceh-13, mab-5 and egl-5 (HOX5/Scr; HOX1/Lab; HOX6-8/Antp; HOX9-13/Abd-B families, respectively) (Hench et al., 2015). Consistently, worms deficient for MES-2, MES-3 or MES-6 (the PRC2 complex) exhibit ectopic HOX gene expression, albeit in a limited number of cells (Ross and Zarkower, 2003). We chose to examine more closely the HOX gene egl-5, because even though it did not make our stringent 4-fold cut-off for derepression, it was derepressed to a low level in all $l s m-8$ replicates $(\log 2 \mathrm{Fc}=0.32)$.

The EGL-5 protein is expressed in the tail regions of both hermaphrodites and males, and is required for aspects of male sexual differentiation, especially in male tail development (Ferreira et al., 1999; Ross and Zarkower, 2003). We used an integrated egl-5::gfp reporter (Ferreira et al., 1999) and checked for derepression in individual cells after knockdown of the LSM2-8 complex, comparing expression in adult males treated with mock RNAi and RNAi against $l s m-7$ and mes- 2 . As reported in Ross and Zarkower, males lacking mes-2 activity displayed ectopic derepression of this reporter in the male tail region (Fig. 4A,B) and occasionally displayed anterior expansions of tail structures (Fig. 4C). Following mes-2 RNAi we also found a few nuclei ectopically expressing this reporter outside the tail/posterior region (Fig. 4D, white arrowheads). Interestingly, knockdown of a functional LSM2-8 complex (lsm-7 RNAi) led to a very similar significant ectopic derepression of the egl-5 HOX reporter (Fig. 4A,B) and also occasionally provoked anterior expansions of the tails (data not shown). Quantitation of cells expressing EGL-5::GFP revealed on average 20 fluorescent cells per worm under mock RNAi conditions, and about 45 cells following either mes-2 or $l s m-7$ RNAi (Fig. 4B). Thus, cell-specific HOX locus repression is LSM2-8 controlled, further linking LSM-8 to the Polycomb pathway.

\section{lsm-8 mutation does not induce transcription from both strands nor alter splicing efficiency}

To understand the mechanism of LSM2-8 silencing, we carried out a careful analysis of strandspecificity by mapping the RNAs recovered in the $l s m-8$ mutant. This showed that derepression occurs exactly over normal gene-coding sequences, without a detectable increase of inaccurate termination or initiation; nor did we detect transcripts from the complementary strand (Fig. S5A). Given that the LSM2-8 complex is known to stabilize and bind U6 snRNA in yeast and plants (Beggs, 2005; Perea-Resa et al., 2012; Zhou et al., 2014) (Fig. 1G), and that the LSM2-8 complex 
co-precipitates both with U6 snRNA (Fig. S6A) and with a factor involved in U6 snRNA stability (Ruegger et al., 2015), we examined the RNA-seq data for splicing defects. Surprisingly, comparison of exon-exon junction reads in $l s m-8 v s$ WT RNA-seq revealed no prominent changes in splicing events, and notably, no intron retention (Fig. S6B). Indeed, out of 134'836 splicing junctions examined, only 18 exon-exon junctions, which mapped to 13 genes, were reproducibly affected $\left(<0.02 \%\right.$; Fig. S6B); none of these was an $l s m-8^{-/}$upregulated gene. Accurate intron splicing is illustrated in Fig. S5. This makes it very unlikely that altered splicing is the source of $l s m-8^{-/-}$mediated derepression.

The RNA-IP done under native conditions confirmed that LSM2-8 complex co-precipitates with U6 snRNA in C. elegans (Fig. S6A), as in other species, and further showed that LSM2-8 can associate with a transcript it regulates. There was no significant association of LSM2-8 with a control transcript that is not regulated by LSM2-8 (Fig. S6A).

\section{LSM2-8 silences gene expression cooperatively with $\mathrm{XRN}-2$}

We next examined potential cofactors of LSM2-8 that might contribute to mRNA level regulation. The first candidate was XRN-2, which we showed could derepress the heterochromatic reporter (Fig. 2), and which has been proposed to work with LSM2-8 in budding yeast (Tharun, 2009). We monitored its role in silencing genomic loci by comparing RNA-seq data from $l s m-8^{-/}$L3 larvae with an existing transcriptome of WT L4 larvae treated with $x r n-2$ RNAi (Miki et al., 2016) (Fig. 5A). $71 \%$ of the genes upregulated by $l s m-8^{-/}$were also upregulated by $x r n-2$ RNAi (Fig. 5A, yellow) and $95 \%$ of those genes are enriched for H3K27me3 (Tables S32-S3). This argues that LSM-8 and XRN-2 likely function on the same pathway with respect to heterochromatin silencing. Nonetheless, a subset of LSM2-8 target genes ( $<33 \%$, in pink) were unaffected by downregulation of $\mathrm{XRN}-2$. It is unclear if those reflect experimental differences or a subset of genes silenced by LSM2-8 through another mechanism. As expected, more genes were affected by $x r n-$ 2 RNAi than by the $l s m-8^{--}$mutation (green), given that XRN-2 is involved in a number of other RNA processing events (Miki and Grosshans, 2013; Miki et al., 2014). Consistent with the fact that XRN-2 has broader functions, $x r n-2$ deletion leads to early larval arrest (Miki and Grosshans, 2013; Miki et al., 2014), which is a more severe phenotype than that observed in $l s m-8$ mutants

(Fig. 2; Fig. S2). Interestingly, we found that genes silenced by $x r n-2$ independently of $l s m-8$ 
(green) were not enriched for H3K27me3 (Fig. S4D), which suggests that XRN-2 itself is not specific for RNA from Polycomb-marked genes, while the LSM2-8 complex is.

To identify additional cofactors that might cooperate with LSM2-8 and XRN-2, we concentrated on factors involved in RNA transcription or processing. Based on earlier work showing that RNA Pol II subunits cooperate with budding yeast LSM1-7 in cytoplasmic RNA decay (Haimovich et al., 2013; Lotan et al., 2007), we examined the effects of two RNA Pol II subunits (rpb-12, rpb-7) and the type II poly(A) binding protein pabp-2 (HsPABPN1 and SpPab2). We find that like $x r n-2$ RNAi, $r p b-12, r p b-7$ and pabp-2 RNAi's derepress the heterochromatic reporter on their own (Fig. 5B,C). This is not true for all genes implicated in RNA metabolism, as exemplified by $c g h-1$ (HsDDX6 and CsDhh1) and pab-2 (HsPABPC1 and CsPab1), two factors involved in RNA regulation that have the same effect as mock RNAi (Fig. 5C). This suggested that RPB-12, RPB7 and PABP-2 may also contribute to silencing the heterochromatic reporter.

To see if these genes act on a common pathway with LSM-8, we performed RNAi against these factors both in a WT and in the $l s m-8^{-/-}$strain, and scored whether their effects were additive or epistatic with the loss of LSM-8. The $l s m-8$ mutant worms were treated with RNAi and scored for GFP derepression in the homozygous $l s m-8^{-/-}$(red pharynx) worms of the next generation (Fig. 5D). We found the down-regulation of $x r n-2$, $p a b p-2 r p b-12$ or $r p b-7$, was completely epistatic with $l s m-8$ deficiency for reporter derepression, as was the $l s m-7$ RNAi control (Fig. 5E,F). RNAi against the Polycomb HMT mes- 2 was additive with $l s m-8$ deletion, albeit less so than either set25 (H3K9me3 HMT) or mes-4 (H3K36 HMT). We conclude that LSM2-8 acts on a pathway of silencing that is dependent on XRN-2-mediated RNA metabolism. The fact that $l s m-8$ and mes-2 are not fully epistatic argues that Polycomb-mediated silencing does not depend entirely on LSM8. This is to be expected, as PRC2 complexes can repress active transcription, while LSM2-8 likely acts post-transcriptionally (Fig. 5E,F; see below).

Given the striking correlation of LSM-8-sensitivity with H3K27me3 (Fig. 3), we next examined if LSM2-8 silencing requires the presence of $\mathrm{H} 3 \mathrm{~K} 27 \mathrm{me} 3$. To this end, we tried to combine the mes2 mutant with the balanced $l s m-8$ deletion, but as both mutations provoke sterility, this was not possible. Moreover, RNAi was extremely inefficient in the mes-2 null background for all clones tested (e.g. lsm-7, ubq-1, let-607). Therefore, we asked instead whether the loss of LSM2-8 alters 
the accumulation of $\mathrm{H} 3 \mathrm{~K} 27 \mathrm{me} 3$ on affected genes. Indeed, quantitative ChIP-qPCR for H3K27me3 on $l s m-8$ upregulated genes had a significant decrease $(>50 \%)$ in H3K27me3 levels in $l s m-8$ vs WT larvae (Fig. 6A), while $l s m-8$-insensitive genes did not. This suggests that the LSM28 complex feeds back to maintain H3K27me3 levels, either directly or indirectly, specifically at the H3K27me3-marked loci that are sensitive to lsm ablation.

\section{LSM-8 and XRN-2 cooperate to promote RNA decay}

The partial feedback on H3K27me3 levels by the LSM2-8 complex on genes it regulates is unlikely to account for the strong $l s m-8^{-/-}$impact on mRNA level, thus we next tested whether the LSM2-8 complex influences mRNA turnover rates. In WT, $l s m-8$ and mes-2 mutant backgrounds we added $\alpha$-amanitin, an inhibitor of RNA pol II and pol III elongation, to L3 stage larvae, and monitored RNA decay over 6 hours by RT-qPCR. The stability of mRNAs from genes known to be sensitive to $l s m-8^{-/}$(Table S2), was compared between the $l s m-8$ mutant, a mes-2 mutant, and WT L3 larvae, normalizing mRNA levels to the $18 \mathrm{~S}$ rRNA, as its synthesis is insensitive to $\alpha$-amanitin (Fig. S7A). The $l s m-8$ sensitive genes do show a delayed rate of decay in the absence of LSM- 8 (Fig. 6B). The rate varied slightly among the three genes monitored (far-3, grl-23 and ZK970.2, Fig. S7), yet all were significantly different from two control genes (eft-3, F08G2.8), which were unaffected by the $l s m-8$ mutation (Fig. S7B). This indicates that the LSM2-8 complex is required to promote the degradation of the transcripts it regulates. Similarly, transcript levels from the $l s m-8$-sensitive genes far-3 and ZK970.2 were strongly upregulated in $x r n-2$ RNAi-treated worms, with a log2 fold change of 4.9 and 4.0, respectively. This suggests that the elevated levels of mRNA detected in $l s m-8^{-/-}$worms stem from RNA stabilization. Importantly, by monitoring RNA decay in the homozygous mes-2 mutant we scored a similar increase in mRNA stability as that found in the lsm-8 mutant, for transcripts sensitive to LSM-8 ablation (Fig. 6B and S7B). This suggests that H3K27me3, or at least MES-2 is required for the LSM8-mediated RNA degradation.

To see whether RNA degradation primarily acts on nascent transcripts (i.e., is co-transcriptional) or occurs post-transcriptionally on mature mRNA, we compared the newly transcribed pre-mRNA levels with those of spliced mRNAs derived from the heterochromatic reporter pkIS1582. We scored the levels of unspliced and spliced mRNA following $l s m-7, x r n-2$ and mes-2 RNAi, and used set-25 RNAi as a control (Fig. 6C). If repression or degradation occurs at the level of transcription, we expect the increase in pre-mRNA and in mRNA to be equal. If spliced mRNA 
levels are higher than the pre-mRNA levels following RNAi, then the drop in mRNA level is likely to be a post-transcriptional event. Quantitative PCR showed that the loss of LSM-7 and XRN-2 showed a much stronger accumulation of mature mRNA over pre-mRNA (Fig. 6C), whereas the loss of the H3K9me HMT SET-25 affected pre-RNA and mRNA levels equally (Fig. 6C). This is consistent with SET-25 playing a role in transcriptional repression, while LSM2-8 and XRN-2 appear to act primarily on mature RNAs. The down-regulation of mes- 2 had an intermediate effect between set-25 and lsm-7 RNAi, consistent with roles in both the targeting of LSM2-8 and reducing transcription (Fig. 6C).

Taken together our data suggest that LSM2-8/XRN-2 is essential for the post-transcriptional degradation of RNAs originating from Polycomb-marked genes. We conclude that facultative heterochromatic silencing is achieved in C. elegans on two levels: one reflects a reduction in transcriptional efficiency, while the second is primarily post-transcriptional, requiring LSM2-8 and XRN-2 to degrade nuclear RNAs prior to cytoplasmic export (Fig. 7).

\section{Discussion}

We have shown in nematodes that a conserved nuclear complex, LSM2-8, contributes to the selective repression of heterochromatic reporters and of a subset of genomic loci bearing the H3K27me3 epigenetic mark, through the post-transcriptional degradation of mRNA. In contrast, a related cytoplasmic complex, LSM1-7, appears to have no role in heterochromatic silencing. Unlike heterochromatin-linked RNA processing pathways in plants and fission yeast, which include the RITS, TRAMP and exosome complexes (Buhler, 2009; Coy and Vasiljeva, 2011; Grewal and Elgin, 2007; Moazed, 2009), the silencing mediated by LSM-2-8 does not target H3K9me3-marked genes specifically and the complex acts independently of the two C. elegans H3K9-specific HMTs. Our data show instead that the LSM2-8 complex specifically reduces the stability of transcripts arising from H3K27me3-tagged genes, mediating their decay by the 5'-3' exoribonuclease XRN-2 (Fig. 7). Genetically, we also implicate the RNA pol II factors RPB-7 and RPB-12, and the type II poly(A) binding protein (PABP-2) in the silencing of the heterochromatic reporter. Importantly, LSM2-8-mediated silencing of endogenous loci occurs almost exclusively at genes carrying $\mathrm{H} 3 \mathrm{~K} 27 \mathrm{me} 3$, as revealed by transcriptomes of mutant L1 and L3 larval stages. Furthermore, as shown by the RNA decay assay in the mes-2 mutant (Fig. 6), it seems to require 
the presence of MES-2 (the C. elegans EZH2 homolog). Given that derepression of the heterochromatic reporter occurred in all somatic tissues of L4 larvae, we argue that LSM2-8mediated silencing is likely to be broadly relevant, because reporter derepression could be monitored in all somatic cells at all stages of development.

Figure 7 illustrates the proposed mode of action, whereby the LSM2-8 complex is targeted either through MES-2 itself, through an unknown ligand of H3K27me3 or through characteristic aspects of the nascent transcripts (e.g. poly-U stretches) to mediate post-transcriptional degradation by $\mathrm{XRN}-2$. This silencing acts in parallel to H3K27me3-mediated transcriptional repression, apparently increasing the robustness to the Polycomb-mediated repression of developmental genes. The conserved nature of the LSM proteins and of the other factors implicated in the LSM28 silencing pathway (XRN-2, MES-2, PABP-2, etc) suggests that this mechanism may be active in other species.

Based on RNAi and genetic epistasis studies, we propose that the RNA decay mediated pathway identified here may involve the type II poly(A) binding protein (PABP-2, (Hurschler et al., 2011) and the RNA Pol II subunits, RPB-12 and RBP-7, although the pathway is independent of DCAP2, XRN-1, LSM-1 (Fig. 1H) and HPL-2 (data not shown). PABP-2 (HsPABPN1 and SpPab2) is particularly interesting, because PABP-2 is nuclear and appears to regulate 3'UTR and poly(A) tail length (Kuhn et al., 2009). Moreover, it binds nascent RNAs early during the elongation step (Beaulieu et al., 2012; Lemieux and Bachand, 2009). Given that the LSM2-8 complex is known to bind to the 3' oligo(U) tail of the U6snRNA (Zhou et al., 2014), as well as the 3' poly $(\mathrm{A}+$ ) tail of nuclear RNAs (Kufel et al., 2004), we hypothesize that PABP-2 may regulate LSM2-8 specificity by modulating the 3'end of mRNAs at H3K27me3-marked domains. In that way, PABP-2 might serve as a "mediator", to link the chromatin state to the transcripts targeted by LSM2-8.

It is unclear whether any molecular criteria other than $\mathrm{H} 3 \mathrm{~K} 27 \mathrm{me} 3$ modification are necessary to target mRNAs for derepression by LSM2-8/XRN-2. We note that the fraction of H3K27me3marked genes that is sensitive to LSM2-8 repression is likely to be higher than that detected by RNAseq of whole larvae. First, if we apply a 1.5-fold change cut-off, instead of $>4$-fold for steady-state mRNA levels in L3 larvae, we find $21 \%$ of all Polycomb-marked genes are upregulated by loss of LSM-8. This is a highly significant fraction, given the fact that the removal of a silencing pathway is usually insufficient to trigger gene expression: a promoter must also be bound by relevant transcription factors to drive RNA PollI-dependent transcription. A second 
factor that has limited our ability to score all $l s m-8$ sensitive transcripts is the fact that our genomic transcriptome analyses had to be done on RNA extracted from whole animals, due to the lethal nature of the $l s m-8$ deletion. Transcripts that are upregulated in a restricted number of cells, or in a specific cell-type, can easily be missed in whole animal RNA libraries, and require detection by sensitive imaging methods that can monitor derepression in a cell-specific manner. This was implemented for the HOX gene egl-5 (Fig. 4), a Polycomb target that is sensitive to $l s m-8$ ablation, even though its upregulation occurred only in $\sim 45$ posterior cells in males (Bender et al., 2004; Hench et al., 2015; Ross and Zarkower, 2003; Soshnikova and Duboule, 2009; Yuzyuk et al., 2009). A similar pattern of egl-5 derepression was scored upon loss of MES-2, the EZH2 homologue, reinforcing the link of LSM2-8 and XRN-2 to Polycomb (Fig. 4, Table S2). From this result, we infer that the extent to which LSM2-8 and XRN-2 regulate developmentally relevant transcripts from H3K27me3-marked loci is likely be more extensive than what whole animal RNA-seq can reveal.

How LSM2-8 recognizes H3K27me3-marked genes is unclear. While LSM proteins do not contain methyl lysine-binding chromodomains, they may recognize specifically modified RNAs or short ribonucleotide motifs. The recognition of RNAs arising from H3K27me3-marked domains thus could entail features acquired during transcription, such as a specific secondary structure, an RNA modification, a modified or alternative caps, variant poly-A or U tails, or may bind a unique RNAbinding factor. We do not yet rule out that there are H3K27me3-binding chromodomain proteins that help target the LSM2-8 complex, although in this case they must act redundantly. Examples of redundant H3K27me3 binding factors include two recently identified H3K27me3 ligands, CEC1 and CEC-6 (Saltzman et al., 2018), whose loss together with ablation of an H3K9me-binding protein, CEC-3, caused a mortal germline phenotype. We note, however, that unlike the LSM genes, RNAi against these chromodomain proteins did not derepress heterochromatin in a genomewide screen (Towbin et al., 2012), nor in a targeted screen that monitored all C. elegans methyl lysine-binding factors by RNAi (Gonzalez-Sandoval et al., 2015). Thus, if they are involved in the LSM2-8 pathway, it must be in a redundant fashion.

PRC1 subunits are not well conserved in C. elegans (Wenzel et al., 2011), however two C. elegans Polycomb-like factors, SOR-1 and SOP-2, have also been shown to be essential to prevent ectopic HOX gene activation and have RNA binding activity (Zhang et al., 2004; Zhang et al., 2006). Such factors might also provide a link between Polycomb targets and the RNA degradation machinery. 
We note that RNAi against RNA Pol II subunits RBP-7 and RBP-12 derepressed the heterochromatic array in a manner epistatic with $l s m-8$ deficiency. Intriguingly, in $S$. pombe the RBP-7 homolog has been implicated in centromeric repeat transcription and RNAi-directed silencing (Djupedal et al., 2005), while in S. cerevisiae, the same RNA Pol II subunit plays a direct role in Pat1/Lsm1-7 mediated mRNA decay in the cytoplasm (Haimovich et al., 2013; Lotan et al., 2007). It is not yet clear what links these various observations, but it should not be excluded that RPB-7 and RPB-12 subunits might mark LSM2-8 regulated transcripts to signal degradation by XRN-2, given their epistatic effect on reporter derepression. A systematic conditional screen of all RNA Pol II subunits and their relationship to $l s m-8$ will be needed to clarify if RNA Pol II subunits participate directly in the silencing pathway described here.

We found no involvement of the decapping enzyme, DCAP-2, or of CGH-1, the closest homolog in C.elegans of the yeast decapping enhancer Dhh1 (Nissan et al., 2010) in the LSM2-8 pathway of degradation, although it is reasonable to expect that a nuclear decapping function might be needed to sensitize target RNAs to degradation by XRN-2. We note that XRN-2 has multiple nuclear functions (Miki and Grosshans, 2013), yet we did not see evidence for aberrant termination events in $l s m-8$ deficient worms, nor are $l s m-8$ sensitive genes enriched for loci that require XRN2 for termination (Miki et al., 2017).

Importantly, we document here by ChIP a drop in H3K27me3 on $l s m-8$-sensitive genes in $l s m-8^{-/-}$ animals, suggesting that the LSM2-8-mediated RNA degradation pathway feeds back to maintain H3K27me3 levels. Interestingly, a recent but still debated suggestion was made that ncRNAs, such as Xist or HOTAIR, or other PRC2 binding RNAs, may help target Polycomb in cis or in trans to target genes (Brockdorff, 2013; Johnson and Straight, 2017; Ringrose, 2017). Understanding how this might relate to the mechanism described here, where an RNA binding and degradation complex contributes to gene silencing and $\mathrm{H} 3 \mathrm{~K} 27 \mathrm{me} 3$ maintenance in worms, is a topic of future research. It is nonetheless highly significant that epigenetic information (H3K27me3) is being sensed by an RNA binding complex, that in turn seems to reinforce the chromatin state (Fig. 7). Thus, despite the fact that LSM2-8 works primarily post-transcriptionally (Fig. 6), the pathway may feed back to stabilize the mark that characterized the source of the transcript it degrades.

Overall, our study shows that facultative heterochromatin silencing in a multicellular organism makes use of a mechanism of selective transcript degradation, and not only of transcriptional 
repression. LSM2-8-mediated gene silencing furthermore links a specific epigenetic state to transcript degradation, adding an additional layer of control over differentiation and development.

\section{Acknowledgements}

The accession number for the RNA-seq data is NCBI Gene Expression Omnibus is GSE92851. Some strains were provided by the Caenorhabditis Genetics Center (CGC), which is funded by NIH Office of Research Infrastructure Programs (P40 OD010440). We thank I. Katiç, the FMI Genomics and Microscopy facilities, P. Zeller and M. Fukushima for technical help, advice and discussion, and T.S. Miki for access to data and discussions. We thank M. Bühler, H. Großhans and W. Filipowicz for discussions and proofreading of the text. The authors acknowledge support of the Novartis Research Foundation, as well as a Marie Curie Intra-European grant (\#PIEF-GA-2010-276589) and Swiss National Science Foundation Marie-Heim Vögtlin grant (\#PMPDP3_151381,\# PMPDP3_168717) to AM; SNF grant (\#310030B_156936) to SMG and support of the NCCR RNA \& Disease to H. Großhans (to FA.).

\section{Author Contributions}

A.M. planned and executed most experiments, evaluated results and wrote the paper; S.M.G. planned experiments, evaluated results and wrote the paper; D.G. analyzed with A.M. the RNAseq data and other genome wide data, C.S analyzed the L1 RNA-seq data, J.P. performed the H3K27me3 ChIP-qPCR experiment and analysis; V.K. performed the gonad staining and analysis and F.A provided help for the $l s m-8$ mutant strain generation.

\section{Author Information}

Affiliation: Friedrich Miescher Institute for Biomedical Research, Basel, Switzerland. Anna

Mattout, Dimosthenis Gaidatzis, Jan Padeken, Christoph Schmid, Florian Aeschlimann, Véronique Kalck and Susan M. Gasser

Faculty of Natural Sciences, University of Basel, Basel, Switzerland. Florian Aeschlimann, Susan M Gasser

Université Paul Sabatier- CNRS UMR 5088, Toulouse France. Anna Mattout Competing financial interests: The authors declare no competing financial interests.

Corresponding Author: Correspondence to Susan M. Gasser 


\section{References}

Ahringer, J., and Gasser, S.M. (2018). Repressive Chromatin in Caenorhabditis elegans: Establishment, Composition, and Function. 208, 491-511.

Au, K.F., Jiang, H., Lin, L., Xing, Y., and Wong, W.H. (2010). Detection of splice junctions from paired-end RNAseq data by SpliceMap. Nucleic acids research 38, 4570-4578.

Beaulieu, Y.B., Kleinman, C.L., Landry-Voyer, A.M., Majewski, J., and Bachand, F. (2012). Polyadenylationdependent control of long noncoding RNA expression by the poly(A)-binding protein nuclear 1 . PLoS genetics 8 , e1003078.

Beggs, J.D. (2005). Lsm proteins and RNA processing. Biochemical Society transactions 33, 433-438.

Bender, L.B., Cao, R., Zhang, Y., and Strome, S. (2004). The MES-2/MES-3/MES-6 complex and regulation of histone $\mathrm{H} 3$ methylation in C. elegans. Current biology : CB 14, 1639-1643.

Brockdorff, N. (2013). Noncoding RNA and Polycomb recruitment. RNA (New York, NY) 19, 429-442.

Buhler, M. (2009). RNA turnover and chromatin-dependent gene silencing. Chromosoma 118, 141-151.

Buhler, M., Haas, W., Gygi, S.P., and Moazed, D. (2007). RNAi-dependent and -independent RNA turnover mechanisms contribute to heterochromatic gene silencing. Cell 129, 707-721.

Chalamcharla, V.R., Folco, H.D., Dhakshnamoorthy, J., and Grewal, S.I. (2015). Conserved factor Dhp1/Rat1/Xrn2 triggers premature transcription termination and nucleates heterochromatin to promote gene silencing. Proceedings of the National Academy of Sciences of the United States of America 112, 15548-15555.

Conway, E., Healy, E., and Bracken, A.P. (2015). PRC2 mediated H3K27 methylations in cellular identity and cancer. Current opinion in cell biology 37, 42-48.

Cornes, E., Porta-De-La-Riva, M., Aristizabal-Corrales, D., Brokate-Llanos, A.M., Garcia-Rodriguez, F.J., Ertl, I., Diaz, M., Fontrodona, L., Reis, K., Johnsen, R., et al. (2015). Cytoplasmic LSM-1 protein regulates stress responses through the insulin/IGF-1 signaling pathway in Caenorhabditis elegans. RNA (New York, NY) 21, $1544-1553$. Coy, S., and Vasiljeva, L. (2011). The exosome and heterochromatin : multilevel regulation of gene silencing. Advances in experimental medicine and biology 702, 105-121.

Djupedal, I., Portoso, M., Spahr, H., Bonilla, C., Gustafsson, C.M., Allshire, R.C., and Ekwall, K. (2005). RNA Pol II subunit Rpb7 promotes centromeric transcription and RNAi-directed chromatin silencing. Genes \& development 19, 2301-2306.

Eberle, A.B., Jordan-Pla, A., Ganez-Zapater, A., Hessle, V., Silberberg, G., von Euler, A., Silverstein, R.A., and Visa, N. (2015). An Interaction between RRP6 and SU(VAR)3-9 Targets RRP6 to Heterochromatin and Contributes to Heterochromatin Maintenance in Drosophila melanogaster. PLoS genetics 11 .

Egan, E.D., Braun, C.R., Gygi, S.P., and Moazed, D. (2014). Post-transcriptional regulation of meiotic genes by a nuclear RNA silencing complex. RNA (New York, NY) 20, 867-881.

Gaydos, L.J., Wang, W., and Strome, S. (2014). Gene repression. H3K27me and PRC2 transmit a memory of repression across generations and during development. Science (New York, NY) 345, 1515-1518.

Golisz, A., Sikorski, P.J., Kruszka, K., and Kufel, J. (2013). Arabidopsis thaliana LSM proteins function in mRNA splicing and degradation. Nucleic acids research 41, 6232-6249.

Gonzalez-Sandoval, A., Towbin, B.D., Kalck, V., Cabianca, D.S., Gaidatzis, D., Hauer, M.H., Geng, L., Wang, L., Yang, T., Wang, X., et al. (2015). Perinuclear Anchoring of H3K9-Methylated Chromatin Stabilizes Induced Cell Fate in C. elegans Embryos. Cell 163, 1333-1347.

Grewal, S.I., and Elgin, S.C. (2007). Transcription and RNA interference in the formation of heterochromatin. Nature 447, 399-406.

Grossniklaus, U., and Paro, R. (2014). Transcriptional silencing by polycomb-group proteins. Cold Spring Harbor perspectives in biology 6 , a019331.

Habacher, C., Guo, Y., Venz, R., Kumari, P., Neagu, A., Gaidatzis, D., Harvald, E.B., Faergeman, N.J., Gut, H., and Ciosk, R. (2016). Ribonuclease-Mediated Control of Body Fat. Developmental cell 39, 359-369.

Haimovich, G., Choder, M., Singer, R.H., and Trcek, T. (2013). The fate of the messenger is pre-determined: a new model for regulation of gene expression. Biochimica et biophysica acta 1829, 643-653.

Hench, J., Henriksson, J., Abou-Zied, A.M., Luppert, M., Dethlefsen, J., Mukherjee, K., Tong, Y.G., Tang, L., Gangishetti, U., Baillie, D.L., et al. (2015). The Homeobox Genes of Caenorhabditis elegans and Insights into Their Spatio-Temporal Expression Dynamics during Embryogenesis. PloS one 10, e0126947.

Hendriks, G.J., Gaidatzis, D., Aeschimann, F., and Grosshans, H. (2014). Extensive oscillatory gene expression during C. elegans larval development. Molecular cell 53, 380-392. 
Ho, J.W., Jung, Y.L., Liu, T., Alver, B.H., Lee, S., Ikegami, K., Sohn, K.A., Minoda, A., Tolstorukov, M.Y., Appert, A., et al. (2014). Comparative analysis of metazoan chromatin organization. Nature 512, 449-452. Johnson, W.L., and Straight, A.F. (2017). RNA-mediated regulation of heterochromatin. Current opinion in cell biology 46, 102-109.

Katic, I., Xu, L., and Ciosk, R. (2015). CRISPR/Cas9 Genome Editing in Caenorhabditis elegans: Evaluation of Templates for Homology-Mediated Repair and Knock-Ins by Homology-Independent DNA Repair. G3 (Bethesda, Md) 5, 1649-1656.

Keller, C., Adaixo, R., Stunnenberg, R., Woolcock, K.J., Hiller, S., and Buhler, M. (2012). HP1(Swi6) mediates the recognition and destruction of heterochromatic RNA transcripts. Molecular cell 47, 215-227.

Kufel, J., Bousquet-Antonelli, C., Beggs, J.D., and Tollervey, D. (2004). Nuclear pre-mRNA decapping and 5' degradation in yeast require the Lsm2-8p complex. Molecular and cellular biology 24, 9646-9657.

Kuhn, U., Gundel, M., Knoth, A., Kerwitz, Y., Rudel, S., and Wahle, E. (2009). Poly(A) tail length is controlled by the nuclear poly(A)-binding protein regulating the interaction between poly(A) polymerase and the cleavage and polyadenylation specificity factor. The Journal of biological chemistry 284, 22803-22814.

Langmead, B., Trapnell, C., Pop, M., and Salzberg, S.L. (2009). Ultrafast and memory-efficient alignment of short DNA sequences to the human genome. Genome biology 10, R25.

Latorre, I., Chesney, M.A., Garrigues, J.M., Stempor, P., Appert, A., Francesconi, M., Strome, S., and Ahringer, J. (2015). The DREAM complex promotes gene body H2A.Z for target repression. Genes \& development 29, $495-500$. Lemieux, C., and Bachand, F. (2009). Cotranscriptional recruitment of the nuclear poly(A)-binding protein Pab2 to nascent transcripts and association with translating mRNPs. Nucleic acids research 37, 3418-3430.

Liu, T., Rechtsteiner, A., Egelhofer, T.A., Vielle, A., Latorre, I., Cheung, M.S., Ercan, S., Ikegami, K., Jensen, M., Kolasinska-Zwierz, P., et al. (2011). Broad chromosomal domains of histone modification patterns in C. elegans. Genome research 21, 227-236.

Lotan, R., Goler-Baron, V., Duek, L., Haimovich, G., and Choder, M. (2007). The Rpb7p subunit of yeast RNA polymerase II plays roles in the two major cytoplasmic mRNA decay mechanisms. The Journal of cell biology 178 , 1133-1143.

Margueron, R., and Reinberg, D. (2011). The Polycomb complex PRC2 and its mark in life. Nature 469, $343-349$. Meister, P., Towbin, B.D., Pike, B.L., Ponti, A., and Gasser, S.M. (2010). The spatial dynamics of tissue-specific promoters during C. elegans development. Genes \& development 24, 766-782.

Miki, T.S., Carl, S.H., Stadler, M.B., and Grosshans, H. (2016). XRN2 Autoregulation and Control of Polycistronic Gene Expresssion in Caenorhabditis elegans. PLoS genetics 12, e1006313.

Miki, T.S., and Grosshans, H. (2013). The multifunctional RNase XRN2. Biochemical Society transactions 41, 825830 .

Miki, T.S., Ruegger, S., Gaidatzis, D., Stadler, M.B., and Grosshans, H. (2014). Engineering of a conditional allele reveals multiple roles of XRN2 in Caenorhabditis elegans development and substrate specificity in microRNA turnover. Nucleic acids research 42, 4056-4067.

Moazed, D. (2009). Small RNAs in transcriptional gene silencing and genome defence. Nature 457, 413-420.

Nissan, T., Rajyaguru, P., She, M., Song, H., and Parker, R. (2010). Decapping activators in Saccharomyces cerevisiae act by multiple mechanisms. Molecular cell 39, 773-783.

Noma, K., Sugiyama, T., Cam, H., Verdel, A., Zofall, M., Jia, S., Moazed, D., and Grewal, S.I. (2004). RITS acts in cis to promote RNA interference-mediated transcriptional and post-transcriptional silencing. Nature genetics 36 , 1174-1180.

Patel, T., Tursun, B., Rahe, D.P., and Hobert, O. (2012). Removal of Polycomb repressive complex 2 makes C. elegans germ cells susceptible to direct conversion into specific somatic cell types. Cell reports 2, 1178-1186. Perea-Resa, C., Hernandez-Verdeja, T., Lopez-Cobollo, R., del Mar Castellano, M., and Salinas, J. (2012). LSM proteins provide accurate splicing and decay of selected transcripts to ensure normal Arabidopsis development. The Plant cell 24, 4930-4947.

Ringrose, L. (2017). Noncoding RNAs in Polycomb and Trithorax Regulation: A Quantitative Perspective. Annu Rev Genet 51, 385-411.

Ross, J.M., and Zarkower, D. (2003). Polycomb group regulation of Hox gene expression in C. elegans.

Developmental cell 4, 891-901.

Saksouk, N., Simboeck, E., and Dejardin, J. (2015). Constitutive heterochromatin formation and transcription in mammals. Epigenetics \& chromatin 8,3 .

Saltzman, A.L., Soo, M.W., Aram, R., and Lee, J.T. (2018). Multiple Histone Methyl-Lysine Readers Ensure Robust Development and Germline Immortality in Caenorhabditis elegans. Genetics 210, 907-923. 
Shin, J.H., Wang, H.L., Lee, J., Dinwiddie, B.L., Belostotsky, D.A., and Chekanova, J.A. (2013). The role of the Arabidopsis Exosome in siRNA-independent silencing of heterochromatic loci. PLoS genetics 9, e1003411.

Shirayama, M., Seth, M., Lee, H.C., Gu, W., Ishidate, T., Conte, D., Jr., and Mello, C.C. (2012). piRNAs initiate an epigenetic memory of nonself RNA in the C. elegans germline. Cell 150, 65-77.

Soshnikova, N., and Duboule, D. (2009). Epigenetic temporal control of mouse Hox genes in vivo. Science (New York, NY) 324, 1320-1323.

St-Andre, O., Lemieux, C., Perreault, A., Lackner, D.H., Bahler, J., and Bachand, F. (2010). Negative regulation of meiotic gene expression by the nuclear poly(a)-binding protein in fission yeast. The Journal of biological chemistry 285, 27859-27868.

Tharun, S. (2009). Roles of eukaryotic Lsm proteins in the regulation of mRNA function. International review of cell and molecular biology 272, 149-189.

Timmons, L., Court, D.L., and Fire, A. (2001). Ingestion of bacterially expressed dsRNAs can produce specific and potent genetic interference in Caenorhabditis elegans. Gene 263, 103-112.

Touat-Todeschini, Shichino, Y., E.Dangin, M., Thierry-Mieg, N., Gilquin, B., L.Lambert, Hiriart, E., Sachidanandam, R., Brettschneider, J., Reuter, M., et al. (2017). Selective termination of lncRNA transcription promotes heterochromatin silencing and cell differentiation. 36, 2626-2641.

Towbin, B.D., Gonzalez-Aguilera, C., Sack, R., Gaidatzis, D., Kalck, V., Meister, P., Askjaer, P., and Gasser, S.M. (2012). Step-wise methylation of histone H3K9 positions heterochromatin at the nuclear periphery. Cell 150, 934-

947.

Towbin, B.D., Meister, P., Pike, B.L., and Gasser, S.M. (2010). Repetitive transgenes in C. elegans accumulate heterochromatic marks and are sequestered at the nuclear envelope in a copy-number- and lamin-dependent manner. Cold Spring Harbor symposia on quantitative biology 75, 555-565.

Trojer, P., and Reinberg, D. (2007). Facultative heterochromatin: is there a distinctive molecular signature? Molecular cell 28, 1-13.

Tucker, J.F., Ohle, C., Schermann, G., Bendrin, K., Zhang, W., Fischer, T., and Zhang, K. (2016). A Novel Epigenetic Silencing Pathway Involving the Highly Conserved 5'-3' Exoribonuclease Dhp1/Rat1/Xrn2 in Schizosaccharomyces pombe. PLoS genetics 12, e1005873.

Vasiljeva, L., Kim, M., Terzi, N., Soares, L.M., and Buratowski, S. (2008). Transcription termination and RNA degradation contribute to silencing of RNA polymerase II transcription within heterochromatin. Molecular cell 29 , 313-323.

Wang, J., Jia, S.T., and Jia, S. (2016). New Insights into the Regulation of Heterochromatin. Trends in genetics : TIG 32, 284-294.

Wang, J.J., Cui, D.Y., Xiao, T., Sun, X., Zhang, P., Chen, R., He, S., and Huang, D.W. (2014). The influences of PRG-1 on the expression of small RNAs and mRNAs. BMC genomics 15, 321.

Wenzel, D., Palladino, F., and Jedrusik-Bode, M. (2011). Epigenetics in C. elegans: facts and challenges. Genesis (New York, NY : 2000) 49, 647-661.

Yamanaka, S., Mehta, S., Reyes-Turcu, F.E., Zhuang, F., Fuchs, R.T., Rong, Y., Robb, G.B., and Grewal, S.I. (2013). RNAi triggered by specialized machinery silences developmental genes and retrotransposons. Nature 493, 557-560.

Yuzyuk, T., Fakhouri, T.H., Kiefer, J., and Mango, S.E. (2009). The polycomb complex protein mes-2/E(z) promotes the transition from developmental plasticity to differentiation in C. elegans embryos. Developmental cell 16, 699-710.

Zeller, P., Padeken, J., van Schendel, R., Kalck, V., Tijsterman, M., and Gasser, S.M. (2016). Histone H3K9 methylation is dispensable for Caenorhabditis elegans development but suppresses RNA:DNA hybrid-associated repeat instability. Nature genetics 48, 1385-1395.

Zhang, H., Christoforou, A., Aravind, L., Emmons, S.W., van den Heuvel, S., and Haber, D.A. (2004). The C. elegans Polycomb gene SOP-2 encodes an RNA binding protein. Molecular cell 14, 841-847.

Zhang, T., Sun, Y., Tian, E., Deng, H., Zhang, Y., Luo, X., Cai, Q., Wang, H., Chai, J., and Zhang, H. (2006). RNAbinding proteins SOP-2 and SOR-1 form a novel PcG-like complex in C. elegans. Development (Cambridge, England) 133, 1023-1033.

Zhou, L., Hang, J., Zhou, Y., Wan, R., Lu, G., Yin, P., Yan, C., and Shi, Y. (2014). Crystal structures of the Lsm complex bound to the 3' end sequence of U6 small nuclear RNA. Nature 506, 116-120.

Zofall, M., Yamanaka, S., Reyes-Turcu, F.E., Zhang, K., Rubin, C., and Grewal, S.I. (2012). RNA elimination machinery targeting meiotic mRNAs promotes facultative heterochromatin formation. Science (New York, NY) 335, 96-100. 
Figure 1 Mattout et al.,

A

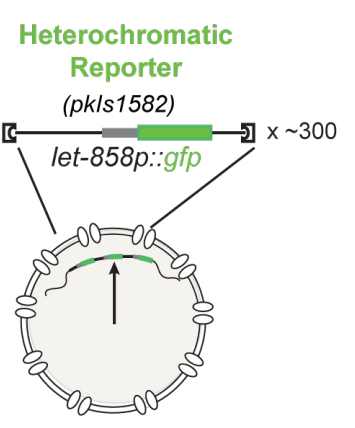

C

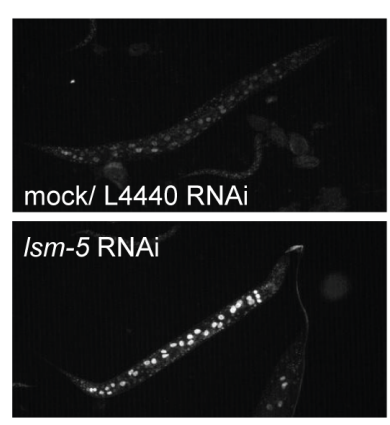

E

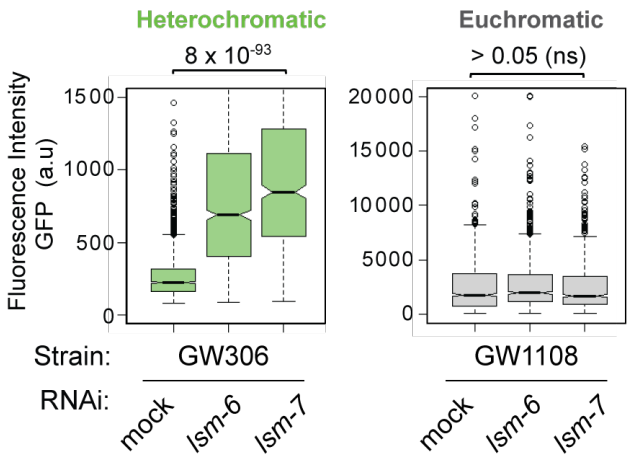

G

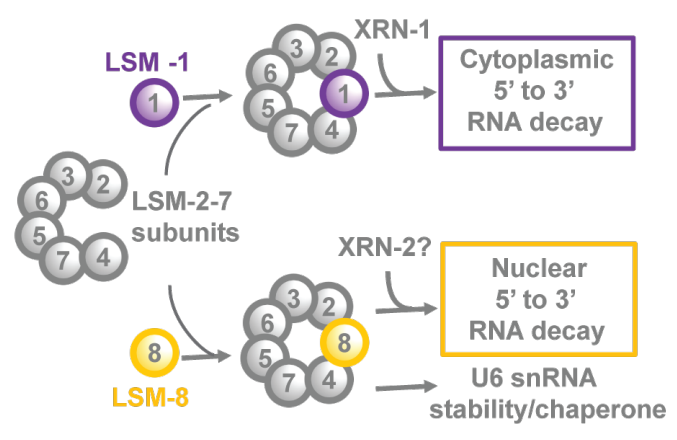

B
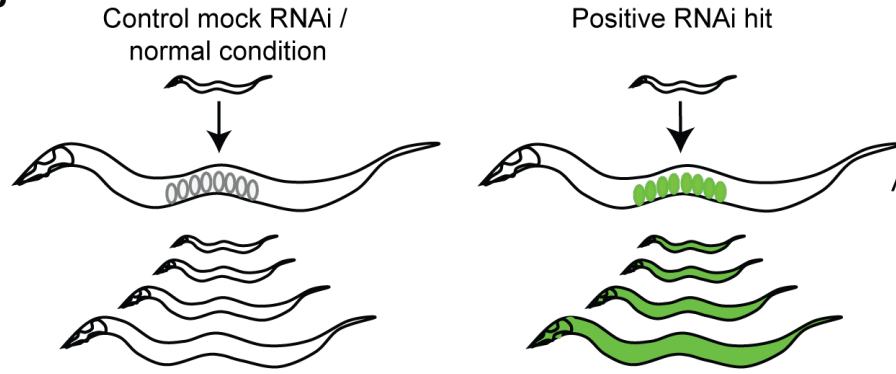

L1 larvae

Adult with embryos

L1

L2

L3

L4

Derepression screen for silenced heterochromatic reporter

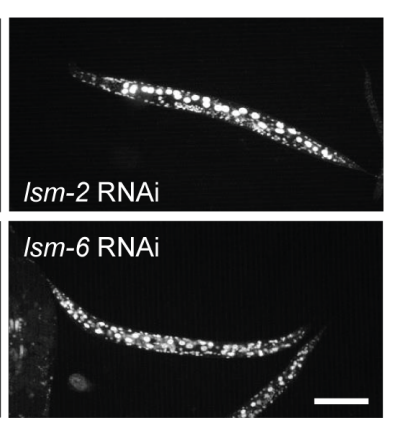

D

Heterochromatic Reporters

Derepression

GW306 (let-858p::gfp::let-858 3'UTR; rol-6(su1006)) V GW76 (baf-1p::.gfp-lacl::let-858 3'UTR; myo-3p::Ifp) X GW299 (tbb-1p::mcherry-lacl::tbb-2 3'UTR; unc-119(+)) GW653 (baf-1p::gfp-Imn-1-Y59C; unc-119(+))

Euchromatic Reporters

GW849 (cec-4p::cec-4-mcherry::cec-4 3'UTR) II

GW1108 (eft-3p::gfp cb-unc-119(+)) II

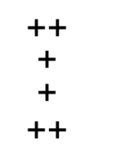

Derepression

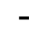

F

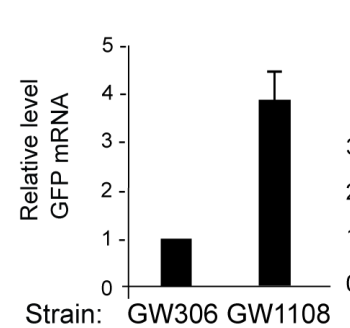

Heterochromatic Reporter

Euchromatic Reporter
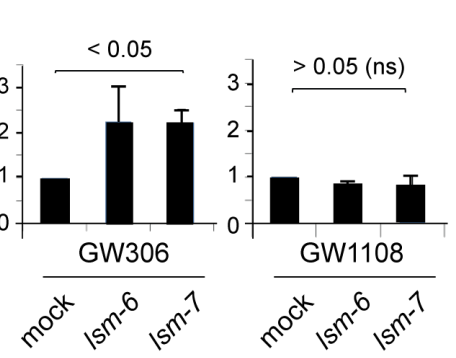

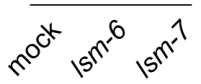

Heterochromatic reporter

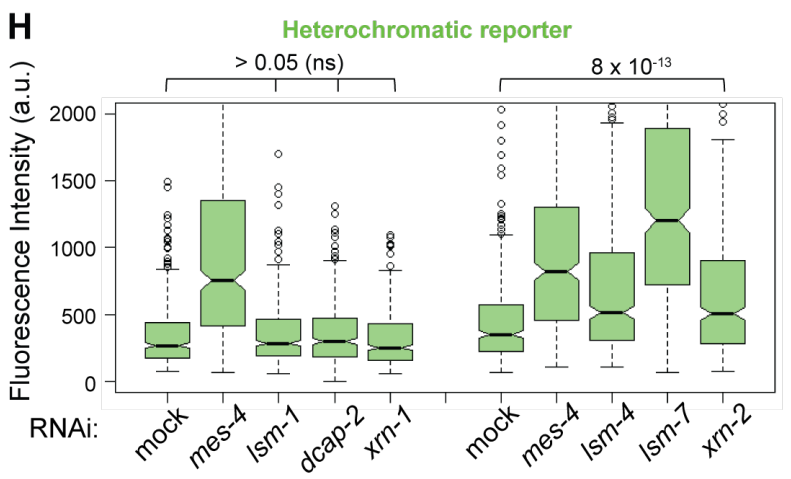


Figure 1: LSM proteins silence heterochromatic reporters, but not euchromatic reporters.

A, Sketch of the integrated, high-copy number heterochromatic reporter pkIs 1582 from strain GW306 used in the genome-wide screen (Towbin et al., 2012). B, Here RNAi-based derepression was monitored in progeny of all stages by increased GFP fluorescence. C, Fluorescence microscopy of pkIs 1582-encoded GFP in L4 larvae with indicated RNAi versus control (mock/L4440). Bar, $100 \mu \mathrm{m}$. D, Heterochromatic and euchromatic reporters scored by eye for derepression $(+,++$ : strong and very strong derepression, respectively) upon LSM RNAi (Table S1). E, Quantitation of derepression in L1 larvae by the worm sorter following indicated RNAi. Box plots of fluorescence intensity in arbitrary units (a.u), with whiskers $=1$ st and 3rd quartiles, black lines: median, black circles outliers. $n=2000,1068,613$ and 875, 111, 1026 . Indicated p-values by Student's t test, (n.s. $=p>0.05)$. F, qPCR analysis of GFP mRNA in L1 larvae as in (E), normalized to his-56 and pmp-3 mRNA. GFP from GW306 strain is set as 1 (left), and mock RNAi conditions are set as 1 (right). $\mathrm{N}=2, \mathrm{n}=3$, bars (mean \pm s.d). G, The two main LSM complexes and functions (Beggs, 2005; Tharun, 2009). H, GFP fluorescence of the heterochromatic reporter (pkIs 1582; GW306) in L1 larvae after RNAi treatment for indicated genes. $n=396$ for each treatment. 
A
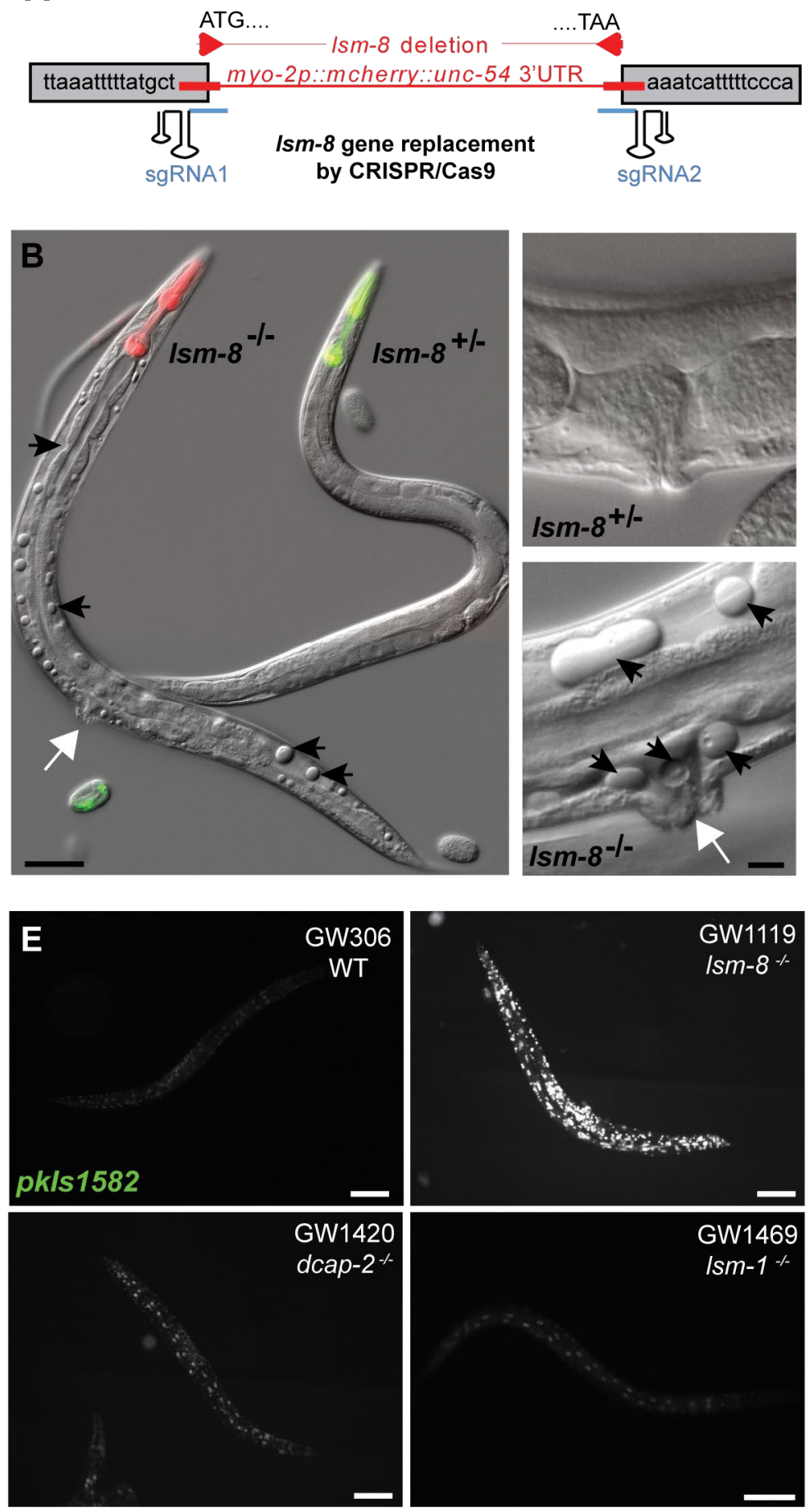

Figure 2 Mattout et al.,
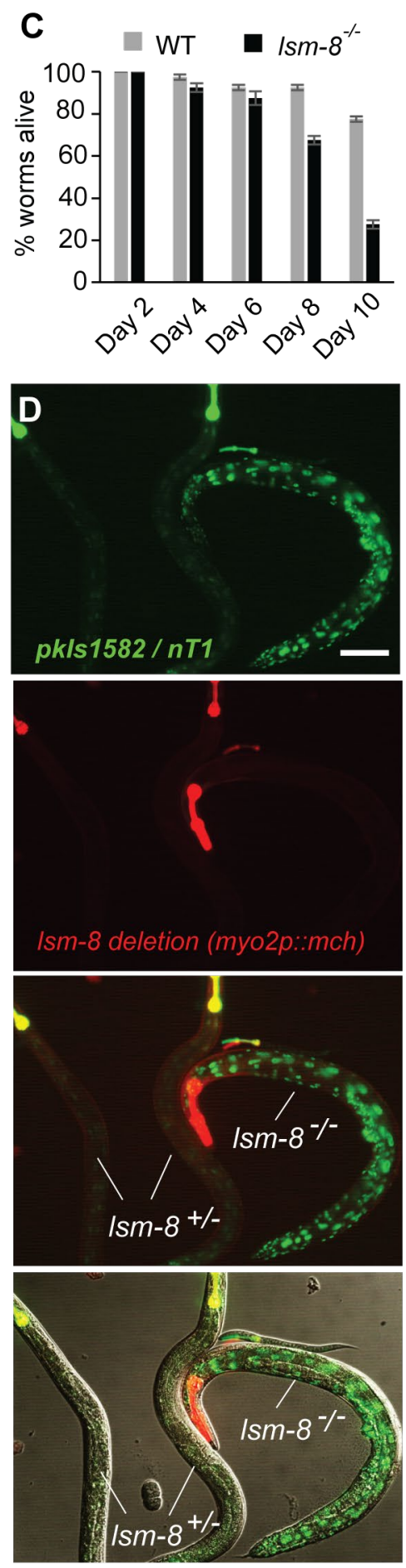


\section{Figure 2: LSM2-8 mediates heterochromatic silencing, and prevents sterility and}

\section{premature death.}

A, Schematic view of the $l s m-8$ deletion/gene replacement created by CRISPR-Cas9. B, Differential interference contrast (DIC) images of young adults (GW1120) merged with pharynx fluorescence to identify genotypes, as in (D). $l s m-8^{-/-}$worms accumulate cavities and vacuoles (black arrows), and protruding vulva (white arrows). Right, enlargement of the vulva region.

Bars, $50 \mu \mathrm{m}$ (left) and $10 \mu \mathrm{m}$ (right). C, Survival assay at $22.5^{\circ} \mathrm{C}$ after hatching shows premature death of $l s m-8^{-/-}$worms. $\mathrm{N}=4, \mathrm{n}=40$ per genotype. Bars (mean \pm s.d). D, View of $l s m-\delta^{+/-}$ (yellow pharynx in merge) and $l s m-8^{-/-}$(red pharynx only) worms carrying the $p k I s 1582$ heterochromatic reporter. Red and green channels are shown separated and merged. Bar, $100 \mu \mathrm{m}$. E, Heterochromatic reporter pkIs 1582 derepression in $l s m-8^{-/}$background compared to the WT, dcap $-2^{-/-}$and $l s m-1^{-/-}$background level. Bars, $100 \mu \mathrm{m}$. 


\section{Figure 3 Mattout et al.,}

A

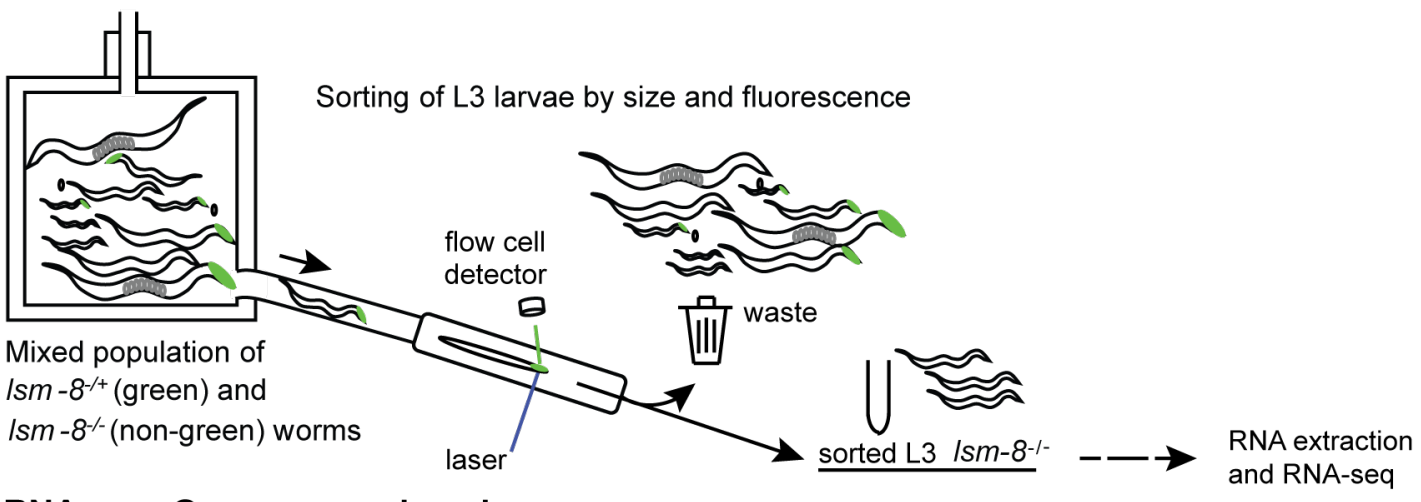

B RNA-seq: Gene expression changes
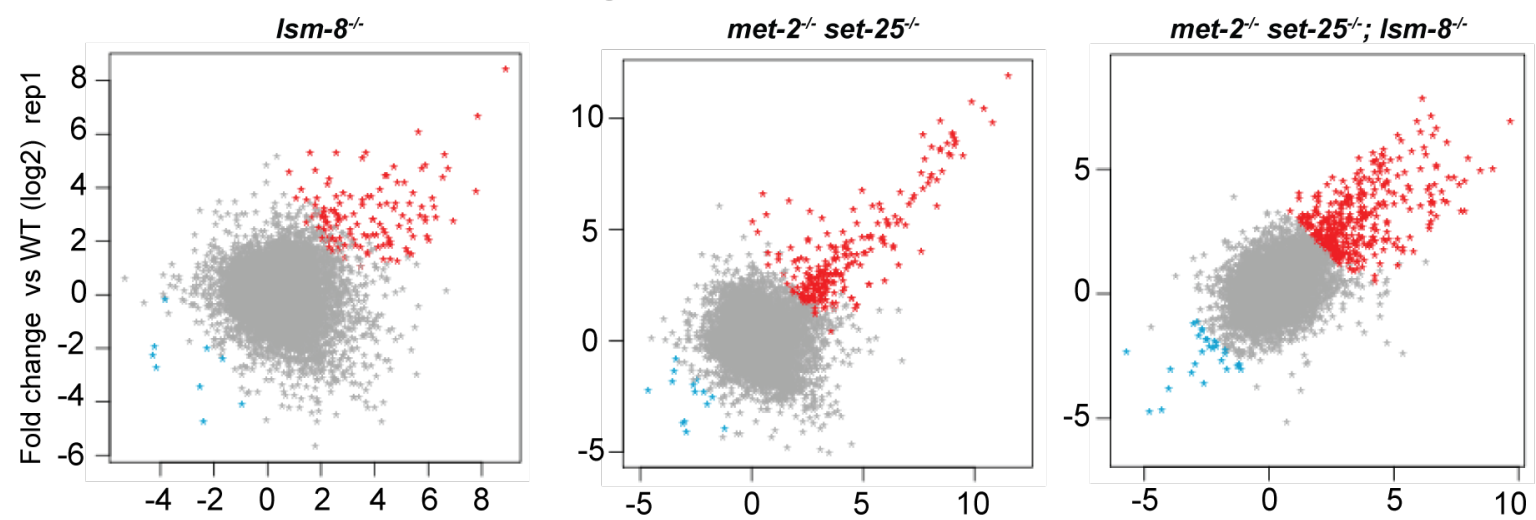

Fold change vs WT (log2) rep2 * up-regulated (FDR $<0.05$ and FC $>4)$ * down-regulated (FDR $<0.05$ and FC $<4)$

C

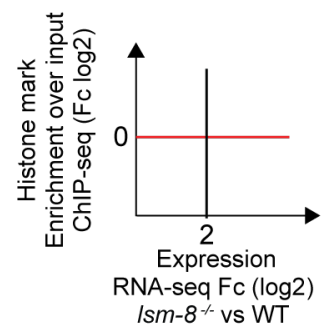

E Abs expression (log2)

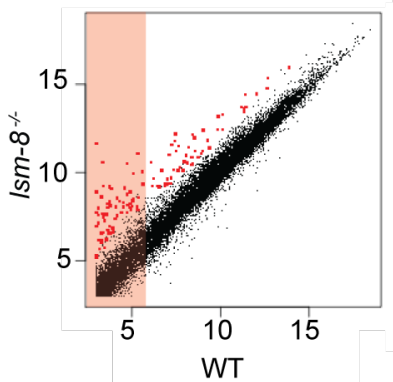

D Euchromatin associated histone marks:
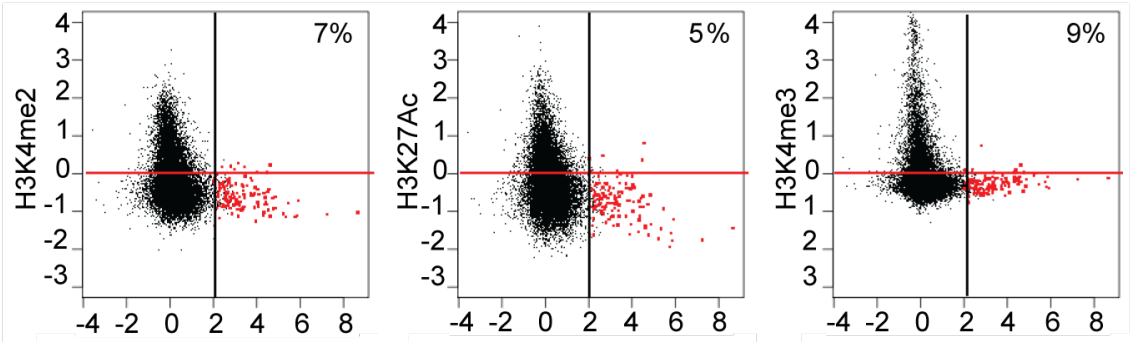

Heterochromatin associated histone marks:
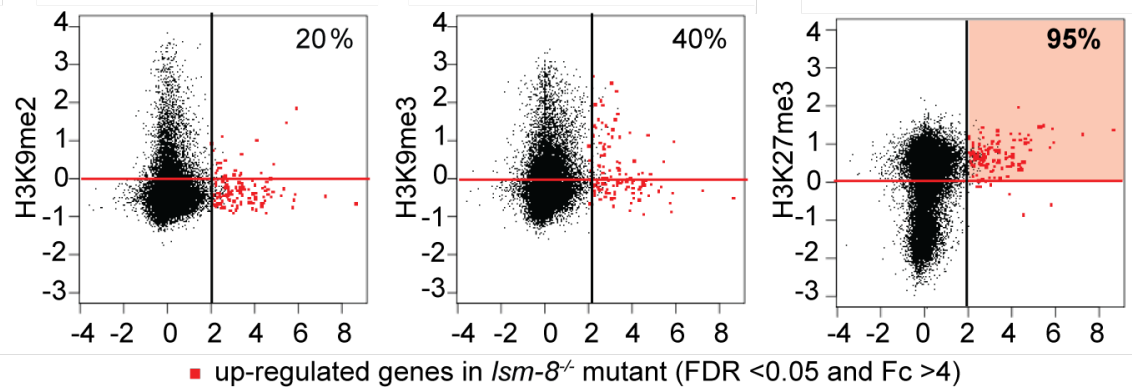


\section{Figure 3: 95\% of genes silenced by LSM2-8 carry the Polycomb mark H3K27me3.}

A, Worm sorting process. L3 worms with the four following genotypes: $l s m-8^{-/-}$; $m e t-2^{-/-} \operatorname{set}-25^{-/}$; triple mutant and WT were sorted and harvested using the same criteria. B, Deletion of $l s m-8$ $\left(l \mathrm{sm}-8^{-/-}\right)$derepresses significantly $>100$ genes $(\mathrm{FDR}<0.05$ and $\mathrm{Fc}>4)$. Relative gene expression profiles are shown as scatter plots, with Fold-change $(\mathrm{Fc})$ in $\log 2(\log 2)$ for two RNA-seq replicas of L3 sorted worms of the indicated genotype versus WT. Each dot corresponds to a gene. C, Scatter plot that compares the average gene expression changes in $l s m-\delta^{-/-}$worms (xaxis in log2, RNA-seq L3 stage) versus enrichment for a histone modification (y-axis in log2, ModEncode data of WT L3 stage). Up-regulated genes (FDR $>0.05$ and Fc $>4$ ) in the $l s m-8^{-/-}$ mutant are in red to the right of the black line, and genes enriched for the histone mark are above the red line (enriched over input). D, Scatter plots as (C), with each dot representing a gene. Upper row, euchromatic marks; lower row, heterochromatin marks. \% indicates genes in upper right zone: LSM-8 regulated and enriched genes for indicated mark. E, Scatter plot of absolute gene expression (normalized reads count, $\log 2$ ) of $l s m-8^{-/-}$versus WT. Red dots as in (D). Values under $6(\log 2)$ are considered to have very low expression (pink shading). 


\section{Figure 4 Mattout et al.,}
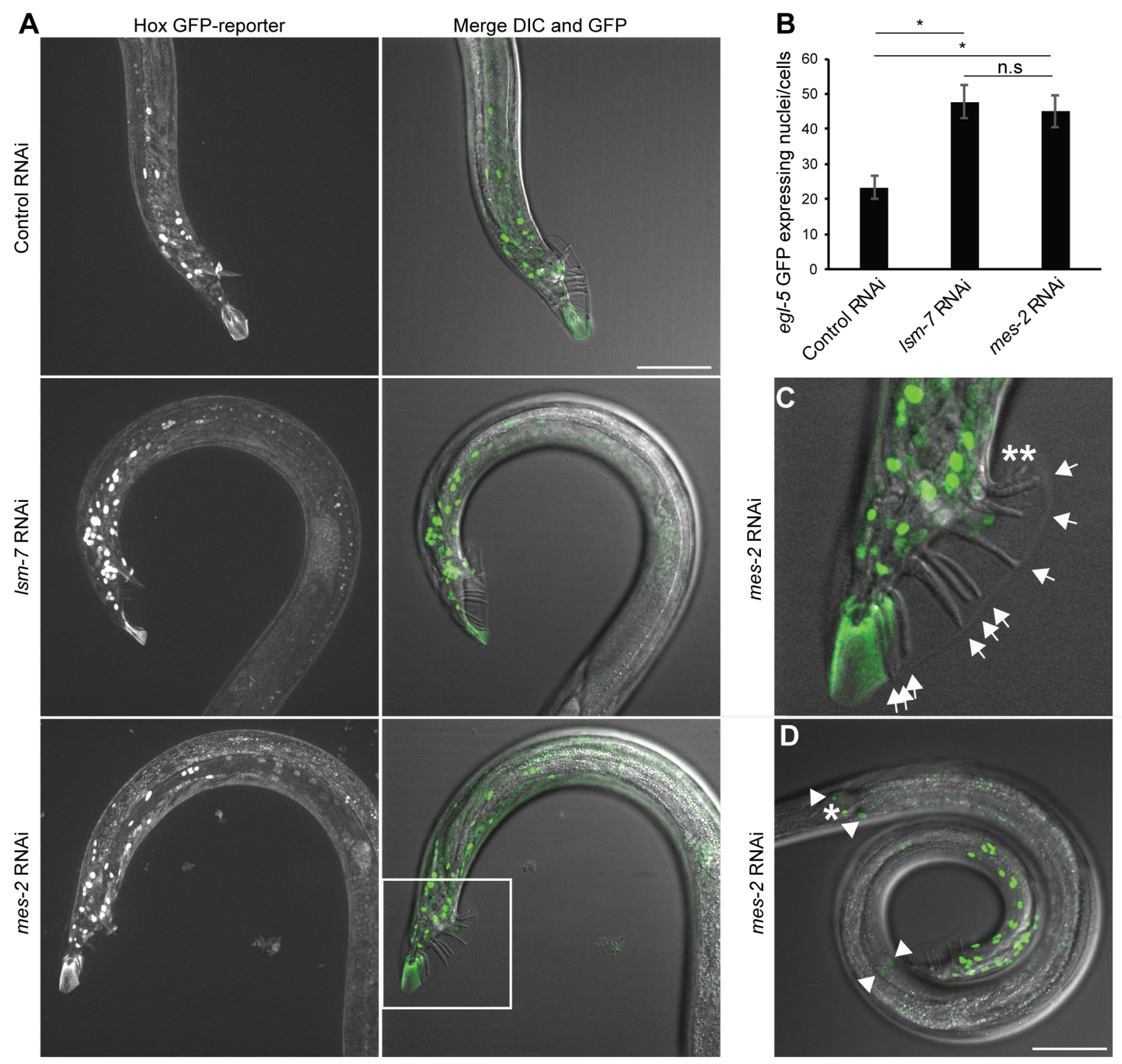

Figure 4: lsm-7, like the EZH2 homolog mes-2, is required to silence the egl-5 Hox gene

A, On the left, Z-projection of confocal images showing the GFP fluorescence of the egl-5 GFP HOX reporter (bxIs13) under Control (mock/L4440) RNAi, lsm-7 and mes-2 (EZH2 homolog) RNAi conditions, in adult males. On the right, merged images of the $\mathrm{Z}$ projection of the GFP signal with the DIC image at the best focal plan to visualize the rays of the male tail. Bar, $50 \mu \mathrm{m}$. B, Quantification of the number of expressing egl-5 GFP nuclei/cells under the indicated RNAi conditions per proximal region of worms. Student's t test p-values: $*<0.001 ; n . s=p>0.5$. N=3; 
$\mathrm{n}=18,19,17$, bars $=$ s.e.m. C, Enlarged male tail inset as in (A) showing the 9 normal rays by arrows and an example of 2 ectopic abnormal rays in mes-2 (asterisks). D, egl-5 GFP derepression is observable mostly in male tail region, as in (A) but a few nuclei ( 0 up to 4 , as shown by the arrowheads) could also exhibit this derepression in other regions of the worm in lsm-7 and mes-2 RNAi conditions. The nucleus indicated by an asterisk express egl-5 GFP in all conditions tested. Bar, $50 \mu \mathrm{m}$. 
Figure 5 Mattout et al.,

A

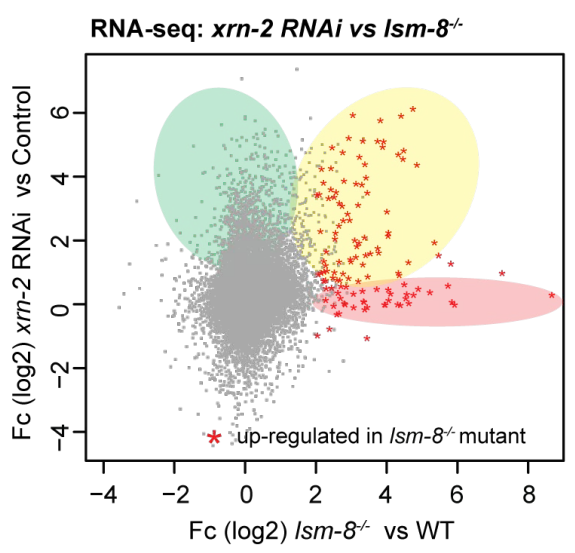

B

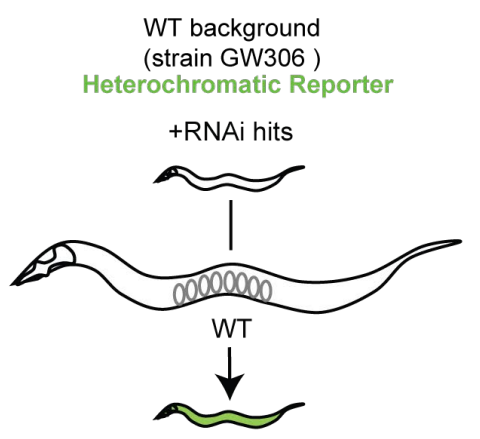

GFP derepression in L1 progeny

C

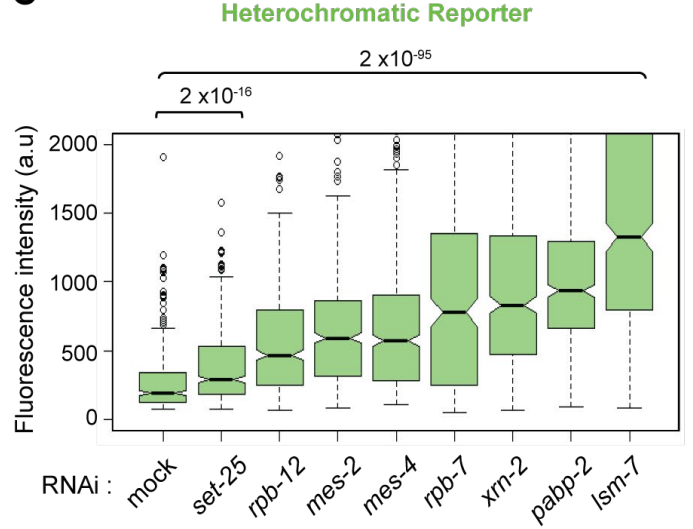

D

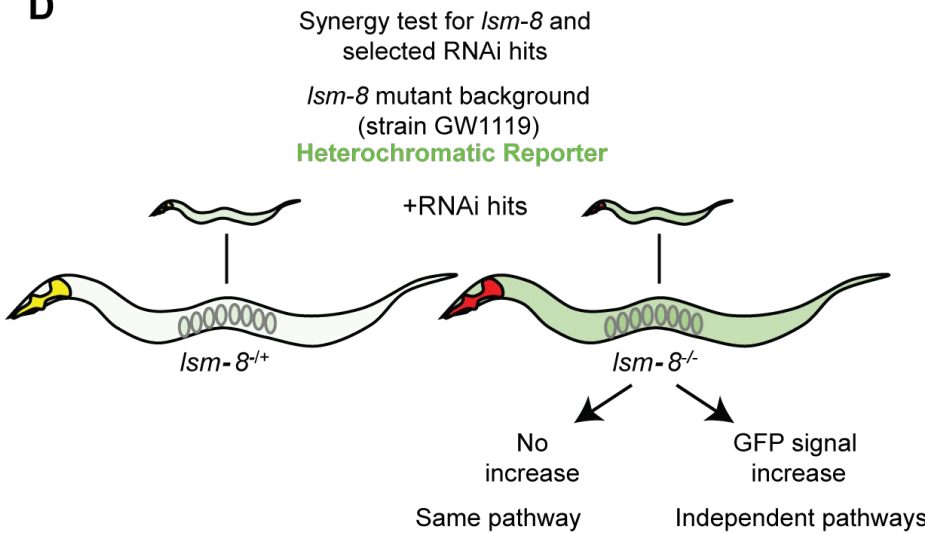

$\mathbf{E}$

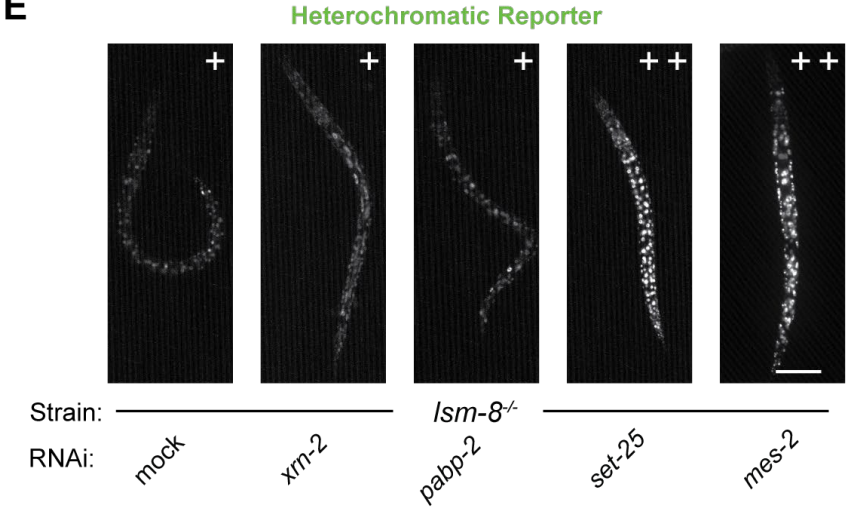

$\mathbf{F}$

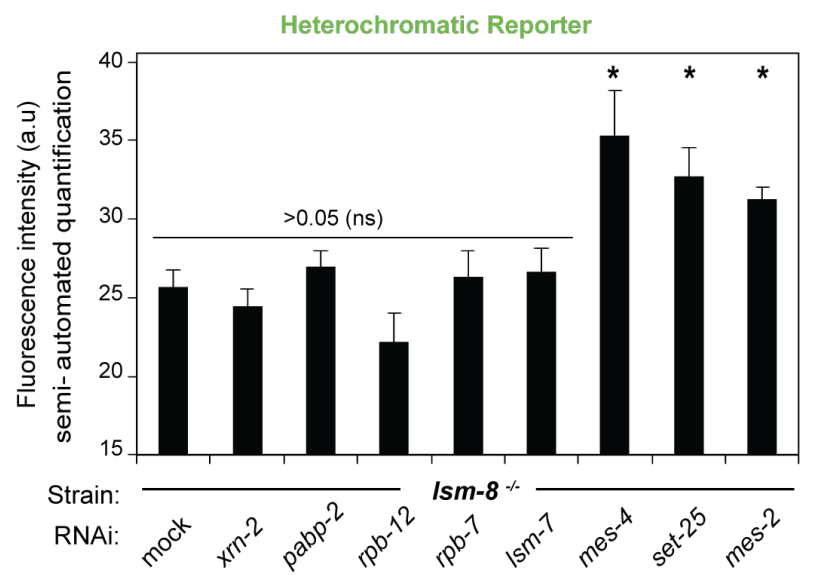

Figure 5: LSM2-8 and XRN-2 work on the same silencing pathway

A, Scatter plot comparing relative gene expression changes of $l s m-8^{-/-}$L3 larvae (this study) and xrn-2 RNAi treated L4 (Miki et al., 2016). Common upregulated genes are shaded yellow; 71\% of genes upregulated in the $l s m-8$ mutant $(\mathrm{FDR}<0.05$ and $\mathrm{Fc}>4$ ) are also upregulated to some 
extent (50\% increase) in $x r n-2$ depleted worms. $l \mathrm{sm}-8^{-/-}$-specific upregulated genes are shaded pink; $x r n-2$ RNAi-specific are shaded green. B, Experimental flow for testing the involvement of additional factors in LSM-8 mediated silencing. RNAi experiments were performed in parallel in WT (B) and lsm-8 mutant (D) backgrounds, from strains GW306 and GW1119, respectively, both carrying the same heterochromatic reporter pkIs 1582. Derepression assay in WT background confirming derepression following RNAi of indicated factors and RNAi efficiency. C, Quantitation of derepression as GFP intensity from the heterochromatic reporter pkIs 1582 in strain GW306, scored in L1 progeny under different RNAi conditions. Fluorescence intensities are displayed as notched box plot. Relevant $\mathrm{p}$ values (t-test) are indicated above. $\mathrm{n}=500,500$, $500,500,500,500,295,500,500$, respectively. D, Scheme for analysis of epistasis of RNAi targets with $l s m-8$ deletion in larvae progeny of heterozygotic $l s m-8+/$ - worms bearing the reporter pkIs1582. E, Fluorescence microscopy of L4 larvae showing same/ non-additive (+) and additive $(++)$ derepression of the reporter pkIs 1582 in $l s m-8^{-/-}$worms under indicated RNAi conditions. Bar, $50 \mu \mathrm{m}$. F, Quantitation of GFP intensity by semi-automated analysis of microscopic images of the reporter pkIs 1582 in $l s m-8^{-/-}$worms under indicated RNAi conditions. $*: \mathrm{p}<0.005$, bars $=$ s.e.m $\mathrm{N}=2, \mathrm{n}=55,45,22,11,10,23,25,25,85$. 
Figure 6 Mattout et al.,

\section{A H3K27me3 ChIP}

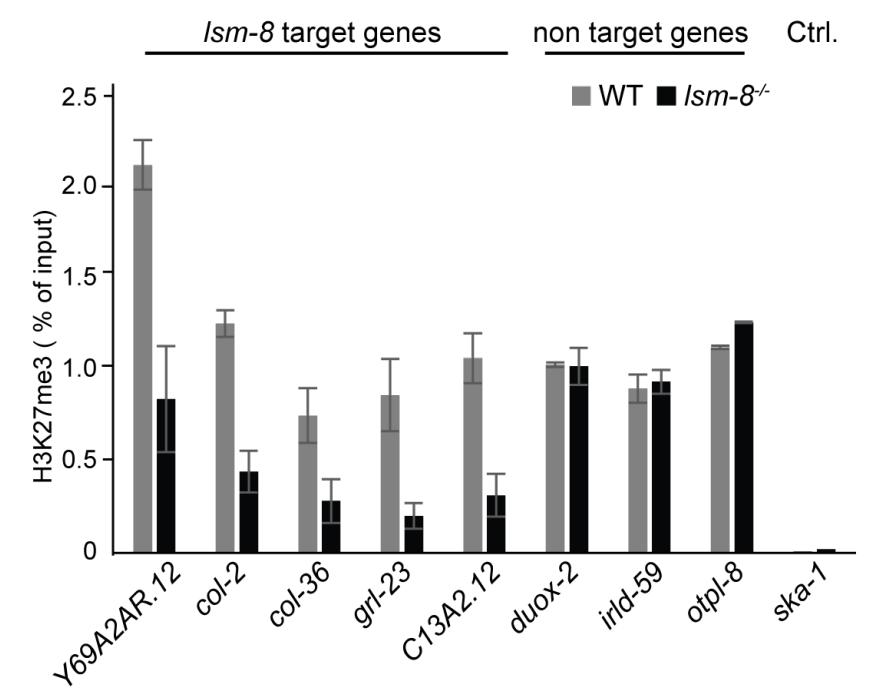

B RNA decay assay
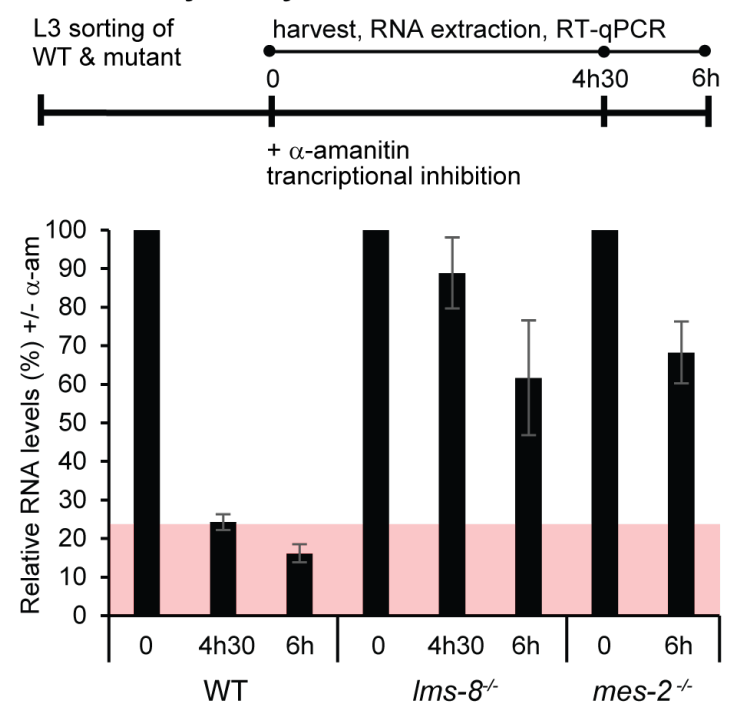

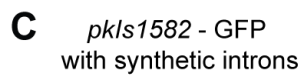

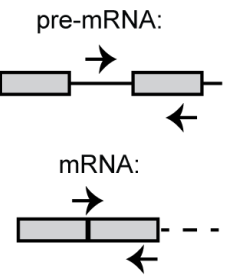

RT-qPCR

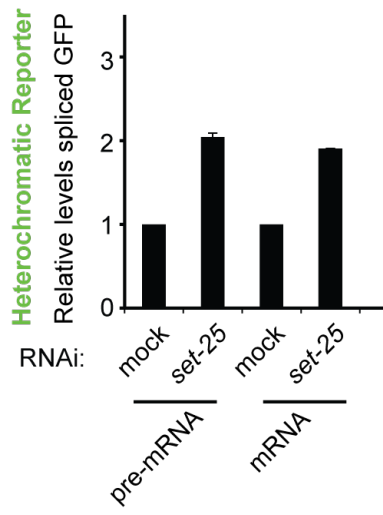

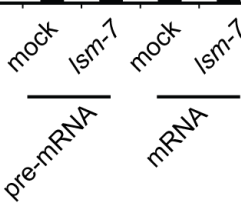

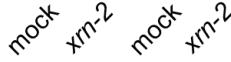

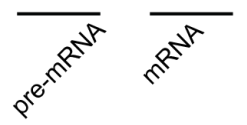

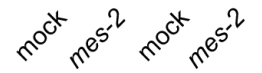

Figure 6: LSM2-8 mediates silencing primarily through RNA degradation.

A, H3K27me3 ChIP-qPCR on target genes in WT and $l s m-8^{-/}$worms. Three categories of genes were assessed. Genes that are upregulated in $l s m-8^{-/}$worms and enriched for H3K27me3 mark in WT (lsm- 8 target genes), genes that are enriched for the H3K27me3 mark in WT but do not change in expression in the $l s m-8$ mutant (non-target genes), and the control ska-1, a gene not enriched for $\mathrm{H} 3 \mathrm{~K} 27 \mathrm{me} 3$ in WT and with no expression change (ctrl). $\mathrm{N}=3, \mathrm{n}=3$, bars = s.e.m. $\mathbf{B}$, WT $l s m-8^{-/}$and $m e s-2^{-/}$worms treated with $50 \mu \mathrm{g} / \mathrm{ml}$ of the transcriptional inhibitor $\alpha$-amanitin 
for indicated times. Levels of transcripts from 3 genes regulated by LSM-8 or two that are not (see individual genes and results in Fig. S7) were tested by RT-qPCR and normalized to 18S rRNA levels. Oh was defined as 100\%. N=3, n=3, bars: s.e.m. C, RNA levels of the pre-mRNA and mRNA of GFP from the heterochromatic reporter pkIs 1582 from the strain GW306 were determined by RT-qPCR and normalized to $p m p-3$ mRNA. The levels on mock RNAi conditions are defined as $1 . \mathrm{N}=2,3,2,3$, respectively; $\mathrm{n}=3$, bars = s.e.m. mes-2 RNAi depletes MES2/PRC2-like and H3K27me3 levels; set-25 RNAi depletes SET-25 and H3K9me3 levels. 


\section{Figure 7 Mattout et al.,}

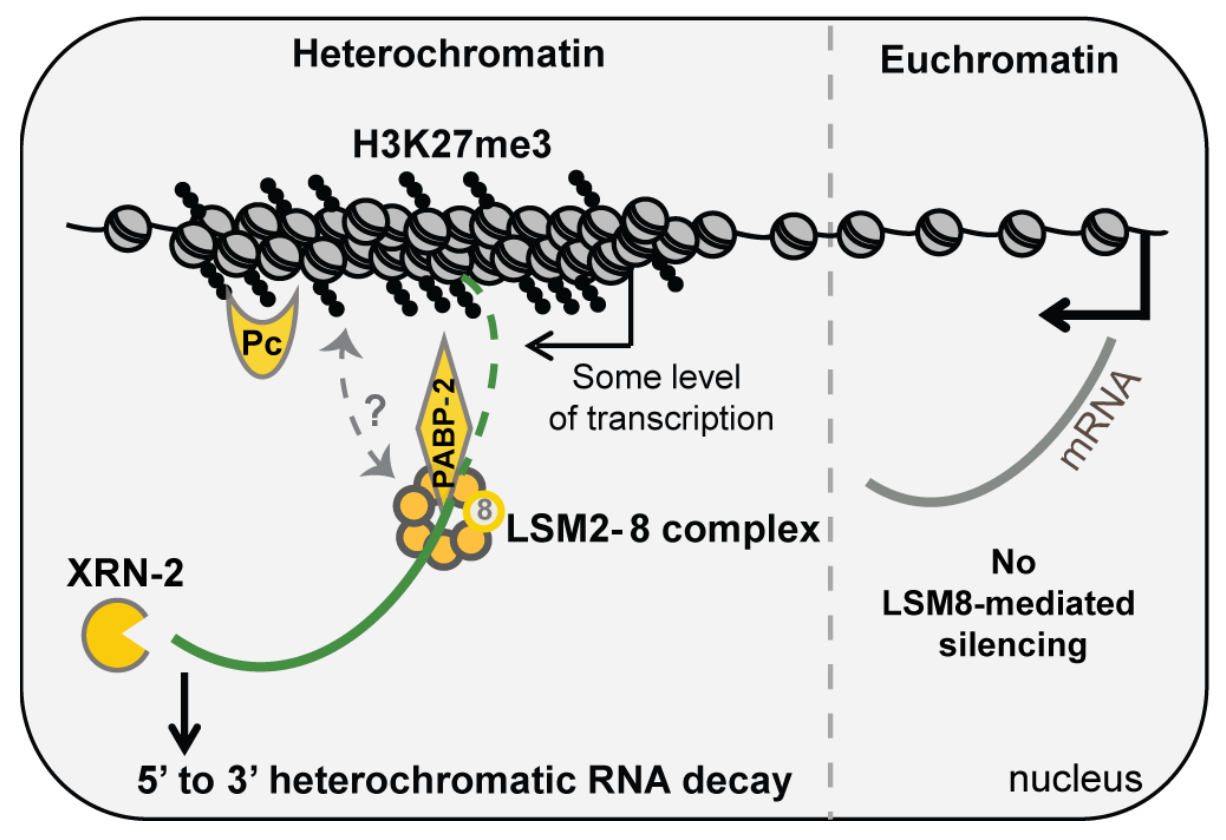

Figure 7: LSM2-8 complex and XRN-2 silence transcripts arising from heterochromatic H3K27me3-enriched domains through RNA degradation.

The LSM-8 mediated silencing pathway makes use of XRN-2 ribonuclease, and may involve other transcript binding factors, such as PABP-2 (HsPABPN1, see Discussion). We hypothesize that RNA arising from $\mathrm{H} 3 \mathrm{~K} 27 \mathrm{me} 3$ genomic regions that are controlled by the LSM2-8 complex may acquire a specific feature during transcription (e.g. a specific structure, RNA modification, 3'UTR, poly-A/U tail, or specific RNA binding protein(s)), that allows recognition and processing by LSM2-8. LSM2-8-mediated silencing also feeds back to regulate H3K27me3 levels on LSM-8-regulated genes, although it is unclear if the interaction with PRC2 or H3K27me3 is direct (dotted arrow). The LSM-2-8-mediated silencing of H3K27me3-bound loci defines a selective post-/co-transcriptional silencing through RNA decay, beyond the transcriptional repression attributed to facultative heterochromatin. 
bioRxiv preprint doi: https://doi.org/10.1101/701581· this version posted July 14,2019 . The copyright holder for this preprint (which was not certified by peer review) is the author/funder, who has granted bioRxiv a license to display the preprint in perpetuity. It is made available under aCC-BY-NC-ND 4.0 International license.

\title{
Supplementary Information
}

\author{
Supplementary Figures S1-S7 \\ Supplementary Tables S1-S5 \\ Experimental Procedures
}




\section{Supplementary Figure 1 Mattout et al.,}

A

\begin{tabular}{c|c|c|c|c} 
& $\begin{array}{c}\text { C. elegans } \\
\text { Protein } \\
\text { length ( a.a.) }\end{array}$ & $\begin{array}{c}\text { H. sapiens } \\
\text { Protein } \\
\text { length ( a.a.) }\end{array}$ & Homology & Identity \\
\hline LSM-1 & 125 & 133 & $68 \%$ & $58 \%$ \\
LSM-2 & 97 & 95 & $94 \%$ & $84 \%$ \\
LSM-3 & 102 & 102 & $85 \%$ & $70 \%$ \\
LSM-4 & 123 & 125 & $89 \%$ & $72 \%$ \\
LSM-5 & 91 & 91 & $90 \%$ & $74 \%$ \\
LSM-6 & 77 & 88 & $76 \%$ & $59 \%$ \\
LSM-7 & 104 & 103 & $81 \%$ & $57 \%$ \\
LSM-8 & 98 & 96 & $78 \%$ & $59 \%$
\end{tabular}

E

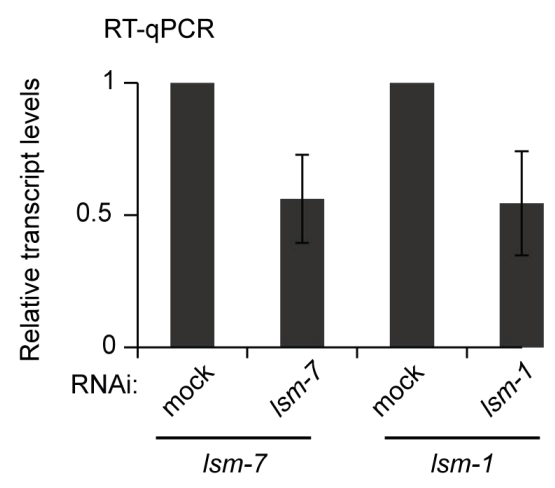

B

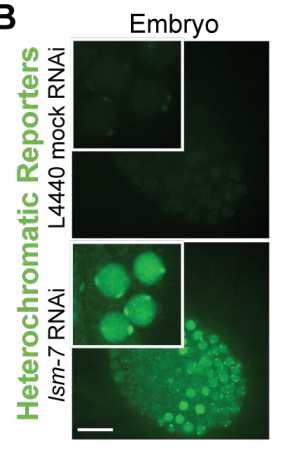

C

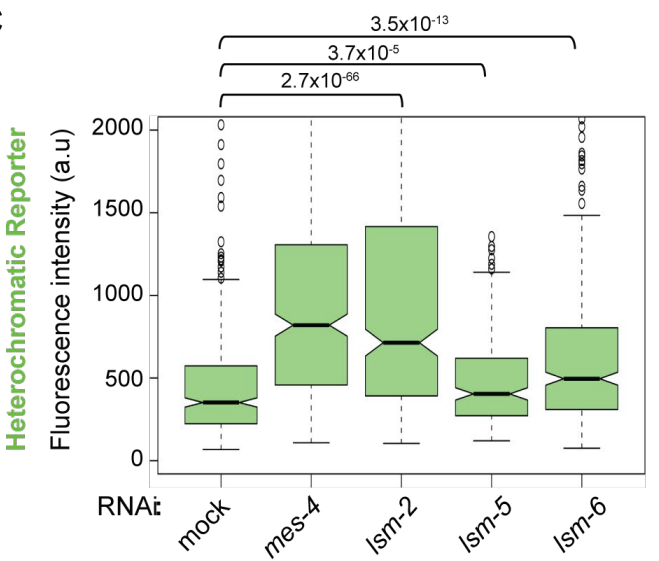

D

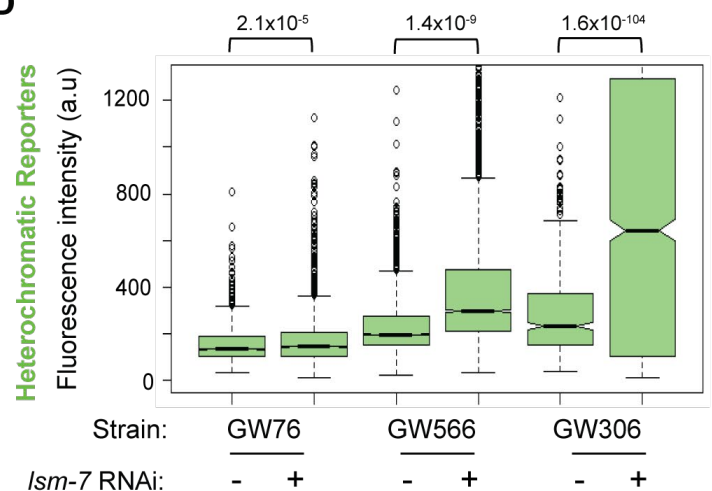

L3
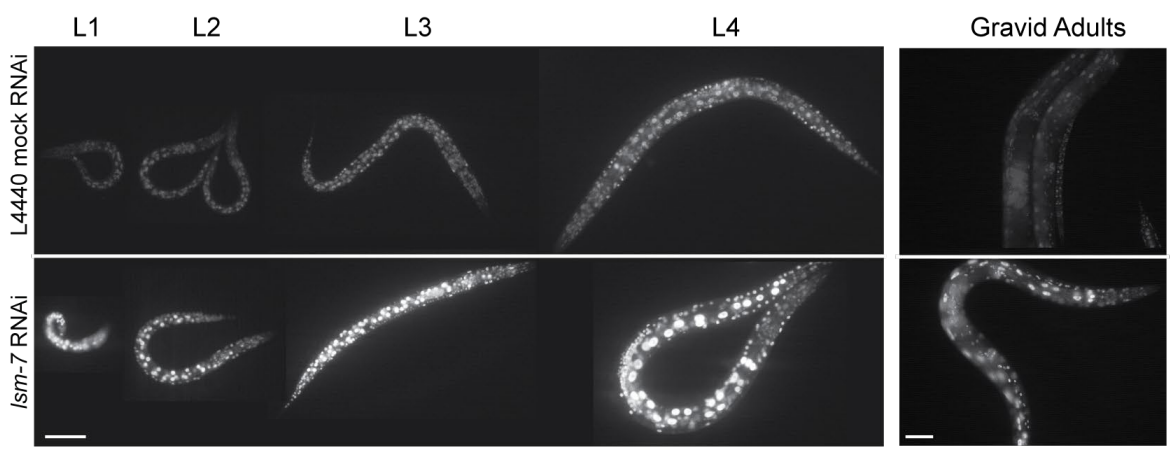

F

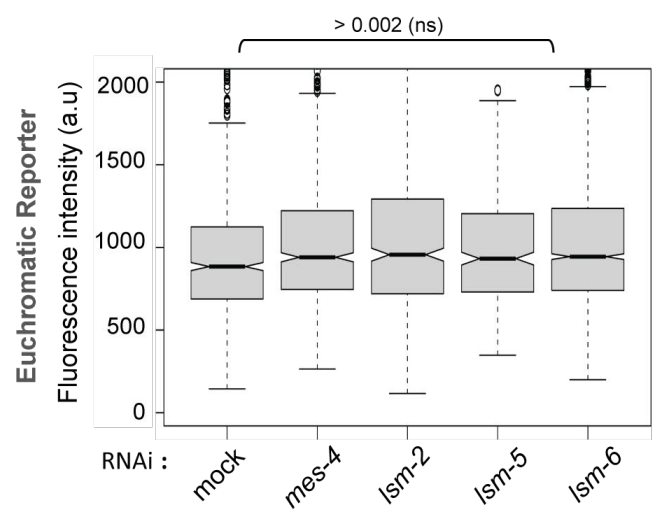

G

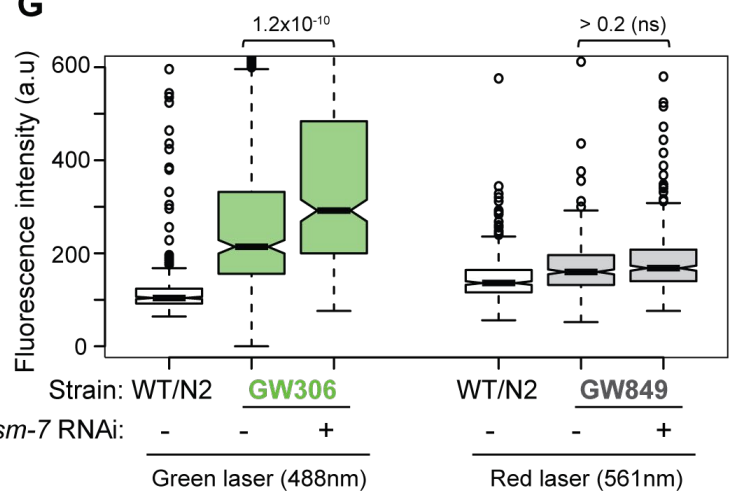




\section{Figure S1: LSM proteins are highly conserved and silence heterochromatic, but not} euchromatic reporters.

A, LSM protein length and conservation between C. elegans and H. sapiens. B, Heterochromatic reporters derepression at all developmental stages. The derepression (GFP live imaging) under lsm-7 RNAi compared to the control RNAi condition is shown for the embryonic stage (heterochromatic reporter from strain GW566, Table S1). Nuclei are enlarged in the inset. Bar, $10 \mu \mathrm{m}$. The derepression is also shown for all larval stages L1-L4, bar: $50 \mu \mathrm{m}$ and gravid adults, bar: $100 \mu \mathrm{m}$ (heterochromatic reporter from strain GW306, Table S1). C, Quantitation of derepression assays. In L1 progeny under gut-2/lsm-2, lsm-5, lsm-6 and control RNAi conditions (mock: negative control and mes-4: positive control), the GFP fluorescence intensity of the heterochromatic reporter pkIS1582 from the strainGW306 was measured by the worm sorter. F2: second generation. $\mathrm{n}=375$ for each condition. The GFP fluorescence intensities are displayed as in Fig. 1. D, Quantitation of derepression of different heterochromatic reporters. Fluorescence intensities are displayed as notched box plot in arbitrary units (a.u), whiskers $=1$ st and 3rd quartiles, black lines: median, black circles outliers. P-values are indicated and were calculated in this and all other plots by pair-wise comparisons with the Student's t-test. In all cases there is a statistically significant reporter derepression upon $l s m-7$ knockdown. $\mathrm{n}=1460,2399,2631$, $3850,634,1855$. E, Confirmation of $l s m-1$ and $l s m-7$ knockdown by RNAi. qPCR analysis of $l s m-7$ and $l s m-1$ mRNA in L1 worms upon mock, $l s m-7$ or $l s m-1$ RNAi treatments, done in parallel to the GFP derepression assay. $l s m-7$ and $l s m-1$ mRNA were normalized to his-56 and its-1 mRNA, and values are expressed relative to the levels in mock RNAi condition. $\mathrm{N}=2, \mathrm{n}=3$, bars $=$ s.d. F, Quantitation of derepression of the euchromatic reporter (GW849) in L1 progeny as in (C). The wmCherry fluorescence intensity (gain 2) of the euchromatic reporter from GW849 strain was quantified. (n.s: not significant). $n=375$ for each condition. $\mathbf{G}$, Same as in (F), except the same gain settings (gain 1) is used both for the fluorescence of the heterochromatic (GW306) and euchromatic (GW849) reporters. The euchromatic reporter fluorescence is lower in this case than the heterochromatic reporter. $n=370$ for all. WT/N2 shows the green and red background fluorescence, respectively, in the absence of the reporter constructs.

\section{Supplementary Table S1: List of strains used in this study}




\begin{tabular}{|c|c|c|c|}
\hline Strain & \begin{tabular}{|l|} 
Original \\
Name / \\
OC \\
\end{tabular} & Genotype & Reference \\
\hline GW1 & $\mathrm{N} 2 *$ & wild-type, Bristol isolate & \\
\hline GW76 & & $\begin{array}{l}\text { gwIs4 [baf-lp::GFP-lacI::let-858 3'UTR; myo- } \\
3 p:: R F P] X\end{array}$ & $\begin{array}{l}\text { (Meister et al. } \\
\text { 2010) }\end{array}$ \\
\hline GW306 & NL2507 & $\begin{array}{l}\text { pkIs 1582[let-858p::GFP:: let-858 3'UTR; rol- } \\
6(\text { su1006)]V }\end{array}$ & $\begin{array}{l}\text { (Towbin et al. } \\
\text { 2012) }\end{array}$ \\
\hline GW566 & & 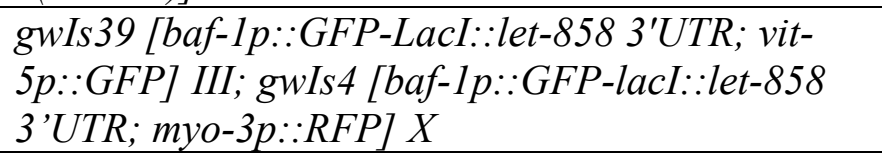 & $\begin{array}{l}\text { (Towbin et al. } \\
\text { 2012) }\end{array}$ \\
\hline GW653 & YG118 & $\begin{array}{l}\text { ygIs[baf-1p::GFP-lmn-1 Y59C; unc-119(+)]; unc- } \\
\text { 119(ed-3) }\end{array}$ & $\begin{array}{l}\text { (Mattout et } \\
\text { al., 2011) }\end{array}$ \\
\hline GW299 & & $\begin{array}{l}\text { gwIs25[tbb-1p::wmCherry-LacI::tbb-2 3'UTR } \\
\text { unc-119(+)] unc-119(ed3) }\end{array}$ & This study \\
\hline GW886 & & $\begin{array}{l}\text { ygIs[baf-1p::GFP-lmn-1 Y59C; unc-119(+)]; set- } \\
\text { 25(n5021) III. met-2(n4256) III }\end{array}$ & This study \\
\hline GW638 & & met-2(n4256) set-25(n5021) III & $\begin{array}{l}\text { (Towbin et al. } \\
\text { 2012) }\end{array}$ \\
\hline GW637 & & $\begin{array}{l}\text { met-2(n4256) set-25(n5021) III; gwIs4 [baf- } \\
\text { Ip::GFP-lacI::let-858 3'UTR; myo-3p }:: R F P] X\end{array}$ & $\begin{array}{l}\text { (Towbin et al. } \\
\text { 2012) }\end{array}$ \\
\hline GW214 & & $\begin{array}{l}\text { hpl-2(tm 1489) III; gwIs4 [baf-1p::GFP-lacI::let- } \\
\text { 858 3'UTR; myo-3p::RFP] X }\end{array}$ & $\begin{array}{l}\text { (Gonzalez et } \\
\text { al., 2015) }\end{array}$ \\
\hline GW468 & & $\begin{array}{l}\text { mes-2(bn11) unc-4(e120)/mnC1 dpy-10e128() unc- } \\
52(e 444) I I ; \text { gwIs } 4 \text { [myo-3::RFP baf-1::GFP lacI } \\
\text { let-858] X. }\end{array}$ & $\begin{array}{l}\text { (Towbin et al. } \\
\text { 2012) }\end{array}$ \\
\hline GW849 & & $\begin{array}{l}\text { gwSil7 [cec-4p::cec-4::WmCherry::cec-4 3'UTR] } \\
\text { II }\end{array}$ & $\begin{array}{l}\text { (Gonzalez et } \\
\text { al., 2015) }\end{array}$ \\
\hline GW1108 & $\begin{array}{l}\text { EG6070 } \\
\text { ocx2 }\end{array}$ & $\begin{array}{l}\text { oxSi221 [eft-3p::GFP + Cbr-unc-119(+)] II (in } \\
\text { ttTi5605); unc-119(ed3) III }\end{array}$ & $\begin{array}{l}\text { (Frøkjær- } \\
\text { Jensen et al., } \\
\text { 2012) }\end{array}$ \\
\hline GW638 & & met-2(n4256) set-25(n5021) III & $\begin{array}{l}\text { (Towbin et al. } \\
\text { 2012) }\end{array}$ \\
\hline GW1109 & $\begin{array}{l}\text { HW1390 } \\
\text { ocx } 4\end{array}$ & lsm-8 (xe17 [myo2p::mcherry::unc54 3'UTR])IV & This study \\
\hline GW1125 & ocx6 & $\begin{array}{l}\text { lsm-8 (xe17 [myo2p:: mcherry::unc54 } \\
\left.\left.3^{\prime} U T R\right]\right) I V / n T 1[q I s 51](I V ; V)\end{array}$ & This study \\
\hline GW1119 & ocx6 & $\begin{array}{l}\text { lsm-8 (xe17 [myo2p::mcherry::unc54 } \\
\text { 3'UTR])IV/nTI[qIs51](IV;V); pkIs1582[let- } \\
\text { 858::GFP rol-6(su1006)]V }\end{array}$ & This study \\
\hline GW1120 & ocx6 & $\begin{array}{l}\text { lsm-8 (xe17 [myo2p:::mcherry::unc54 3'UTR])IV; } \\
\text { pkIs1582[let-858::GFP rol-6(su1006)]V }\end{array}$ & This study \\
\hline GW1148 & ocx6 & 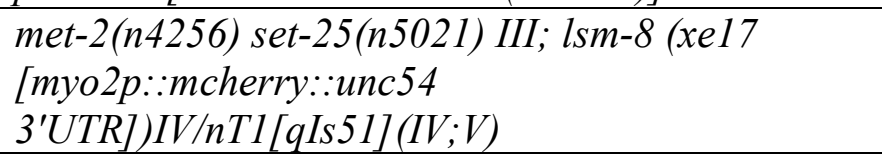 & This study \\
\hline
\end{tabular}




\begin{tabular}{|c|c|c|c|}
\hline GW923 & $\begin{array}{l}\text { VC2663* } \\
\text { ocx } 5\end{array}$ & $l s m-5(o k 3431) \mathrm{V} / n T 1[q I s 51](I V ; V)$ & This study \\
\hline $\begin{array}{l}\text { GW925/ } \\
\text { GW1082 }\end{array}$ & $\begin{array}{l}\text { VC904* } \\
\text { ocx } 5\end{array}$ & $e e a-1 \& g u t-2(g k 407) V / n T 1[q I s 51](I V ; V)$ & This study \\
\hline GW931 & ocx6 & $\begin{array}{l}\text { eea-1\&gut-2(gk407) V/nT1[qIs51](IV;V);gwIs4 [ } \\
\text { myo-3::RFP baf-1::GFP lacI let-858]X }\end{array}$ & This study \\
\hline GW933 & ocx6 & $\begin{array}{l}\text { lsm-5(ok3431) V/nT1[qIs51](IV;V); gwIs4 [ myo- } \\
3:: R F P \text { baf-1::GFP lacI let-858] X }\end{array}$ & This study \\
\hline GW1080 & $\begin{array}{l}\text { VC2785* } \\
\text { ocx } 5\end{array}$ & $\begin{array}{l}l s m-4 \& a d a-2(o k 3151) / m \ln 1[m l s 14 d p y- \\
10(e 128)] I I .\end{array}$ & This study \\
\hline GW1004 & & gwEx81[WRM062D_B06::gfp::3xFlag] & This study \\
\hline GW 1420 & & $\begin{array}{l}\text { dcap-2 (tm2470)/nT1 IV; pkIs1582[let-858::GFP } \\
\text { rol-6(su1006)] V }\end{array}$ & This study \\
\hline GW 1500 & & $\begin{array}{l}l s m-1(\mathrm{tm} 3585) / \mathrm{mln} 1[\mathrm{mls} 14 \mathrm{dpy}-10(\mathrm{e} 128)] \mathrm{II} ; \\
\text { pkIs1582[let-858::GFP rol-6(su1006)] V}\end{array}$ & This study \\
\hline GW1613 & EM599 & him-5(e1490) V; lin-15B\&lin-15A(n765) X; bxIs 13 & CGC \\
\hline
\end{tabular}

*CGC: Caenorhabditis Genetics Center

OCx 4 : out-crossed 4 times 


\section{Supplementary Figure 2 Mattout et al.,}
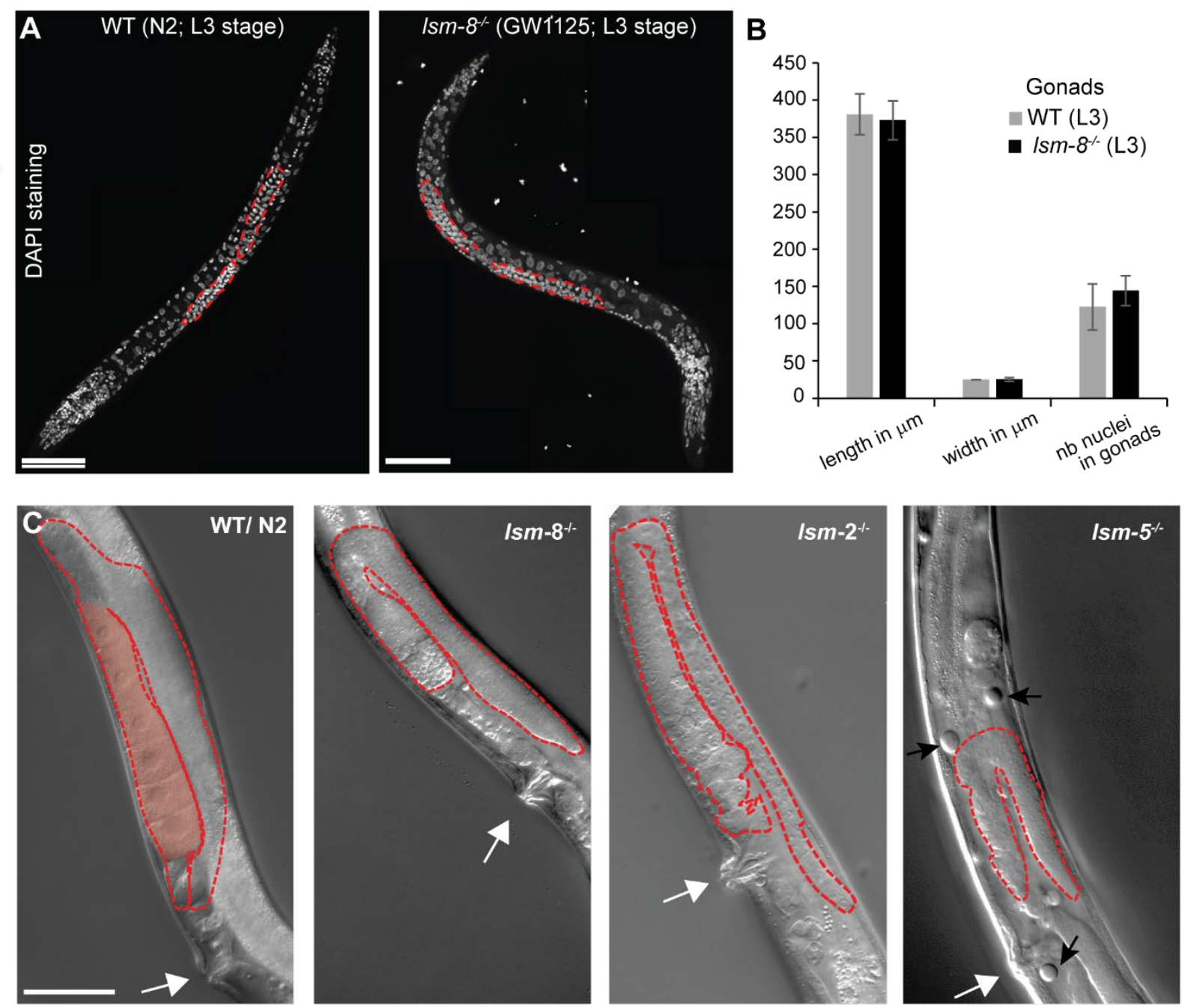

D Ism-7 RNAi (L4)
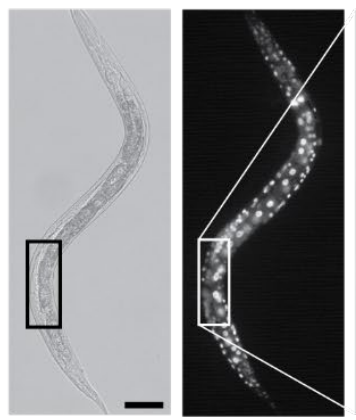

\section{$\mathbf{F}$}

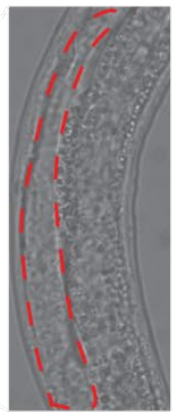

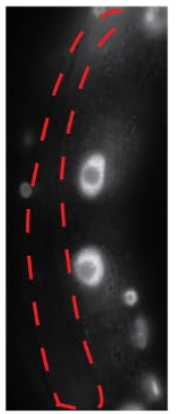

$1 s m-8 \%(L 4)$

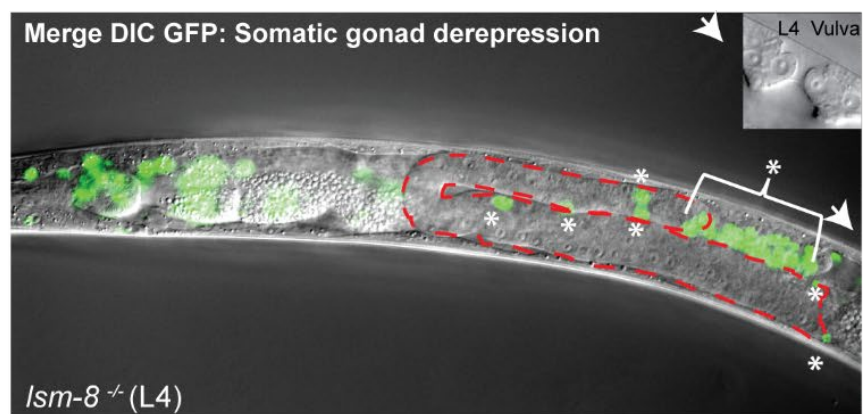

G $1 s m-8^{-/ 2}$ mutant control RNAi

Ism-8\%mutant cSr-1 RNAi
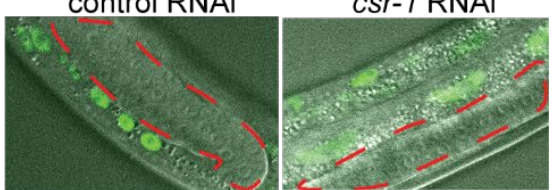


\section{Supplementary Figure S2: $l s m-8^{--}$mutant worms are 100\% sterile but developing gonads resemble WT through $\mathrm{L3}$ and $\mathrm{L} 4$ stages.}

A, Z-projection of confocal images showing fixed DAPI staining of a WT (N2) worm, at L3 stage. Gonad arms are highlighted by the red dashed line and same to right with a $l s m-8^{-/-} \mathrm{L} 3$ larva (GW1125). Bars, $50 \mu \mathrm{m}$. B, Quantification of the length, width and gonad nuclei count from the DAPI staining of L3 WT and $l s m-8-/-$ larvae. $\mathrm{n}=8$. C, DIC image of a WT young adult (YA) worm with a normal general anatomy and normal gonad (red dashed line) with oocytes (pink shading). The white arrow indicates the vulva as in YA. DIC image of $l s m-8^{-/}, l s m-2^{-/-}$and $l s m-5^{-/-}$YA worms. The gonad (red dashed line) has no forming oocytes and has an abnormal composition of cells at that stage. Black arrows indicate the presence of vacuoles. Bar, $50 \mu \mathrm{m}$. D, Heterochromatic reporter (pkIs1582) derepression in WT background from strain GW306 following $l s m-7$ RNAi in a L4 larva. The enlargement to the right shows the gonad (red dashed line) with germ cells which are not derepressed. Bar, $50 \mu \mathrm{m}$. E, Merge DIC and live GFP microscopy of $l s m-8$ mutant carrying the heterochromatic reporter $p k I s 1582$ (GW1119), at the L4 larvae stage as confirmed by the vulva in the inset. The derepression of the reporter in the gonad is not in germ cells, but does occur in the somatic gonad cells marked with asterisks: DTC (distal tip cells), gonadal sheath, spermathecal cells. F, Z-projection of confocal images showing the nuclear GFP derepression of the heterochromatic reporter pkIs 1582 (GW1119) in nearly all if not all somatic cells of an $l s m 8^{-/}$worm. Bar, $50 \mu \mathrm{m}$. G, GFP and DIC merged images at a single focal plan showing the optimal view of germ cells (inside dashed red line), which are not derepressed in $l s m 8^{-/-}$worm $(\mathrm{GW} 1119)$ even treated with RNAi against piRNA factors such as csr-1. 


\section{Supplementary Figure 3 Mattout et al.,}

A Developmental timing of RNA-seq samples

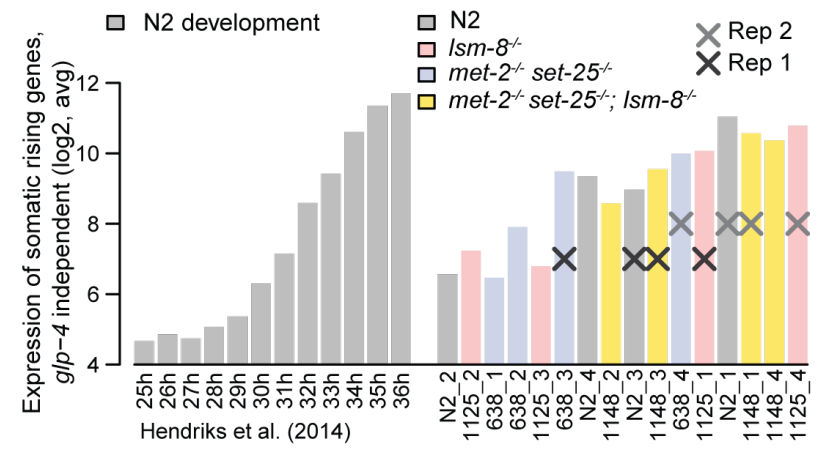

B RNA-seq: Relative gene expression changes
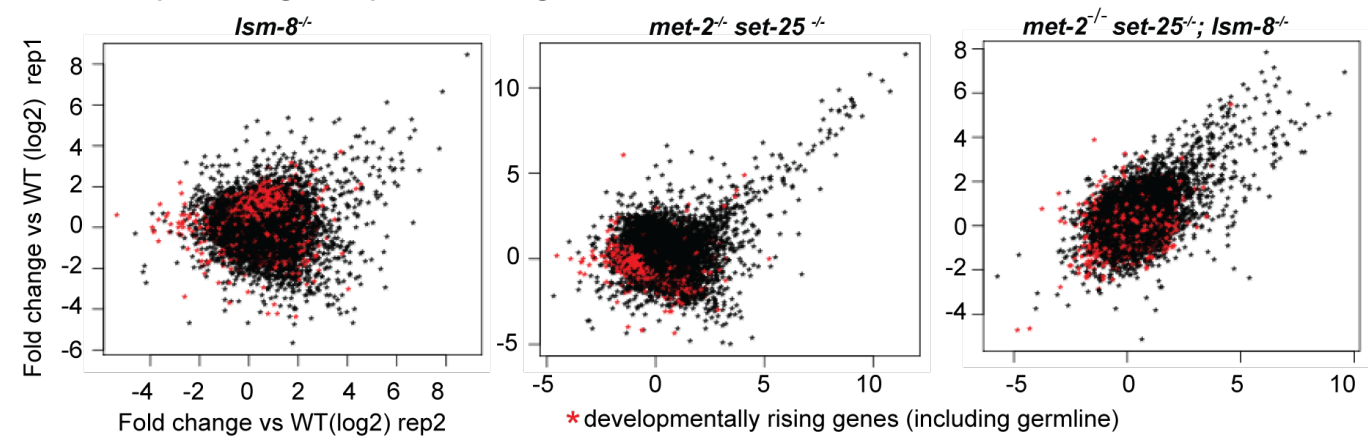

C

RNA-seq: $m e t-2 \%$ set-25\% vs Ism-8 $\%$

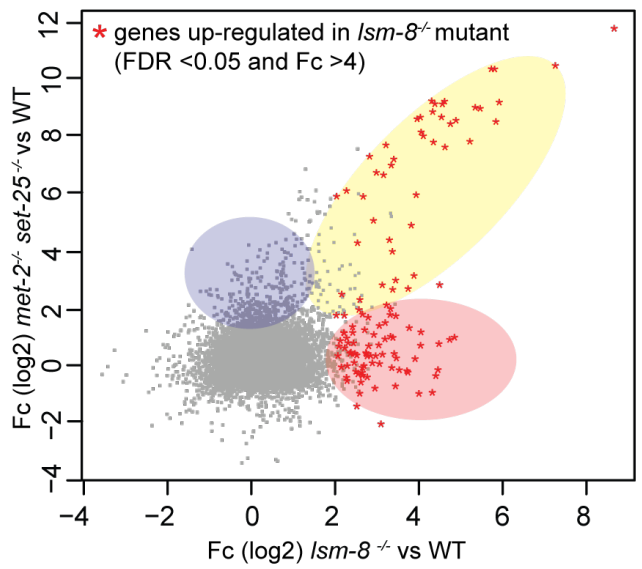

D

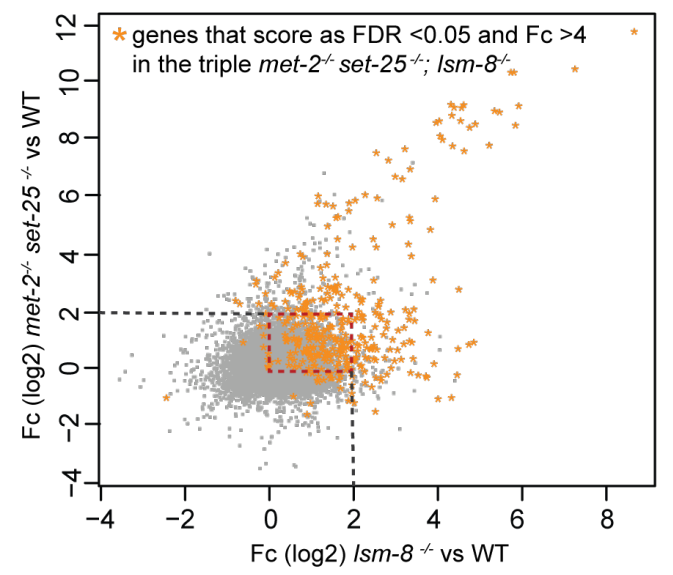

E

$\mathbf{F}$
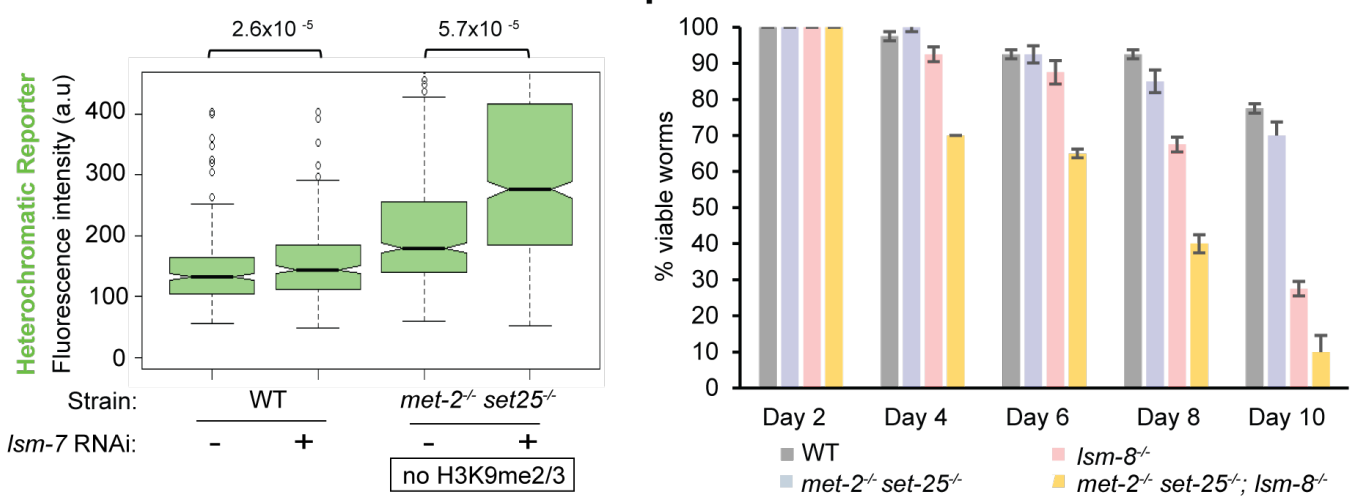


\section{Supplementary Figure S3: LSM2-8 mediated silencing is independent of H3K9 methylation.}

A, Gene expression data were collected over a time course at $25^{\circ} \mathrm{C}$ and the average expression of germline genes that were found to increase during this time course is plotted in the left part (Hendricks et al., 2014). This analysis allowed us to compare our samples to the average expression of germline genes and somatic genes that increase naturally within L3 larval stage (see Experimental procedures). Samples from the four different genotypes matched by developmental timing were selected accordingly and assigned to replica 1 and replica 2.

Bioinformatics analysis was pursued with these samples. B, Relative gene expression profiles as scatter plots. Fold-change $(\log 2)$ in gene expression of two replicas of RNA-seq from sorted L3 worms of $l s m-8^{-/}$, met- $2^{-/-}$set- $25^{-/}$and the triple $\left(l s m-8^{--}\right.$, met- $2^{-/}$set-2 $\left.25^{-/}\right)$mutant versus WT. Each dot corresponds to a gene. Red dots are rising genes, genes with increased expression level during the time course described (Hendriks et al., 2014), which do not change significantly in any of the mutant strains. $\mathbf{C}$, Scatter plot comparing the relative gene expression between the $l s m-8$ (x axis) and the met-2 set-25 double mutant (y axis). Common upregulated genes are shaded yellow; $36 \%$ of genes upregulated in the $l s m-8$ mutant (FDR $<0.05$ and $\mathrm{Fc}>4$ ) are also upregulated $(\mathrm{FDR}<0.05$ and $\mathrm{Fc}>4)$ in the met-2 set-25 mutant. $l s m-8^{-/-}$-specific upregulated genes are shaded in pink; met- $2^{-/-}$set- $25^{-/-}$specific are in blue. D, Comparison of the $l s m-8$ and met-2 set-25 mutants RNA-seq data, as in (C), overlaid by the set of genes that are upregulated (FDR $<0.05$ and $\mathrm{Fc}>4$ ) in the triple mutant met-2 set-25; lsm-8 (orange stars). The dotted red square highlights genes for which the repression pathways are clearly additive: the orange dots indicate genes that are highly derepressed $(>4 \mathrm{Fc})$ in the triple mutant, but mildly $<4 \mathrm{Fc}$ derepressed in either the $l s m-8$ or met- 2 set-25 mutant as shown in this graph. E, Quantitation of derepression of GFP expressed from the gwIs 4 heterochromatic reporter in L1 progeny in WT and met-2 set-25 mutant genotypes, respectively from strains GW76 and GW637, after control or lsm-7 RNAi, displayed as in Fig S1. n= 339, 1004, 426, 673. F, Survival assay as in Figure 2. The $m e t-2^{-/-}$set- $25^{-/} ; l s m-8^{-/}$worms die prematurely compared to the $l s m-8^{-/-}$mutant. $\mathrm{N}=4, \mathrm{n}=40$ worms per genotype, bars $=$ s.d. 


\section{Supplementary Figure 4 Mattout et al.,}

A

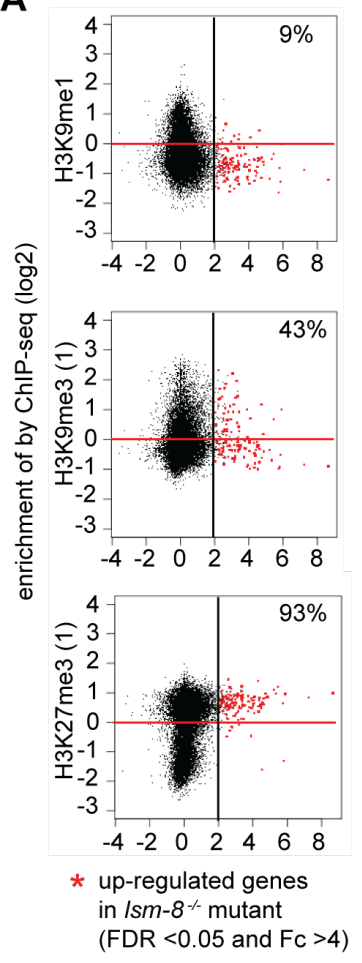

E RNA-seq: L1 stage

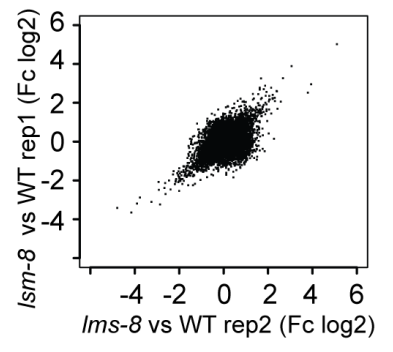

B RNA-seq: Absolute gene expression

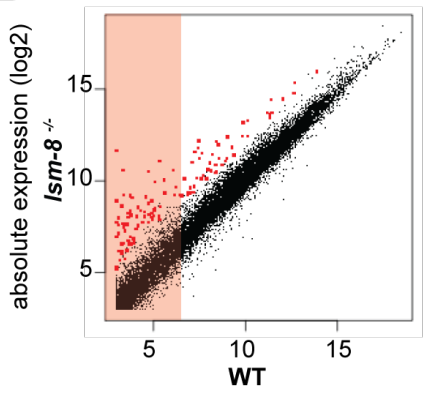

absolute expression (log2)

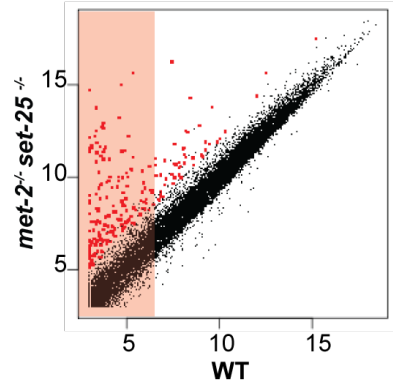

* up-regulated genes in $/ s m-8 \%$ mutant $($ FDR $<0.05$ and Fc $>4)$
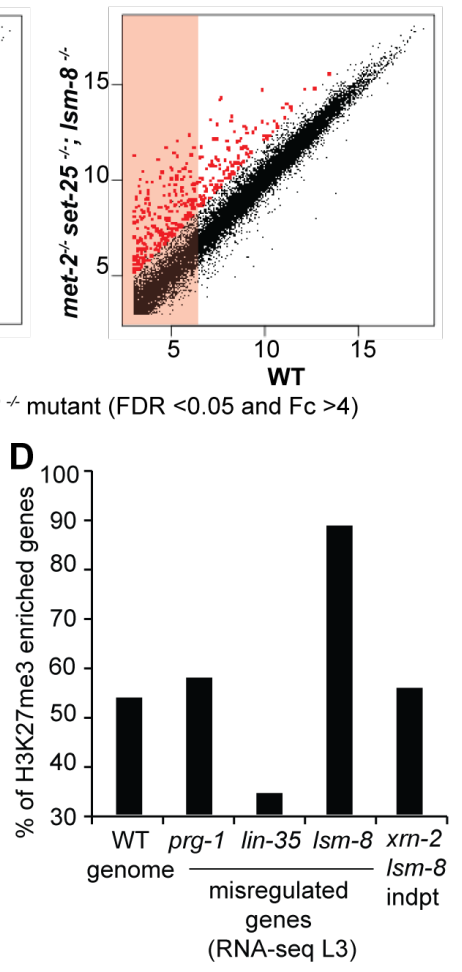

F Euchromatin associated histone marks:
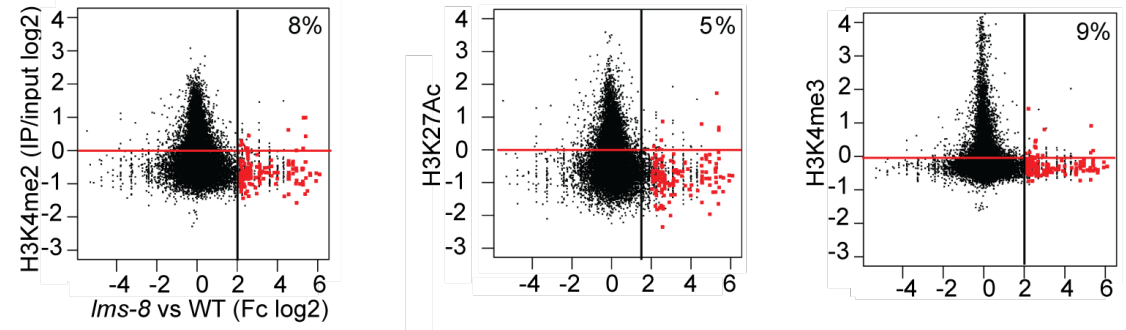

Heterochromatin associated histone marks:
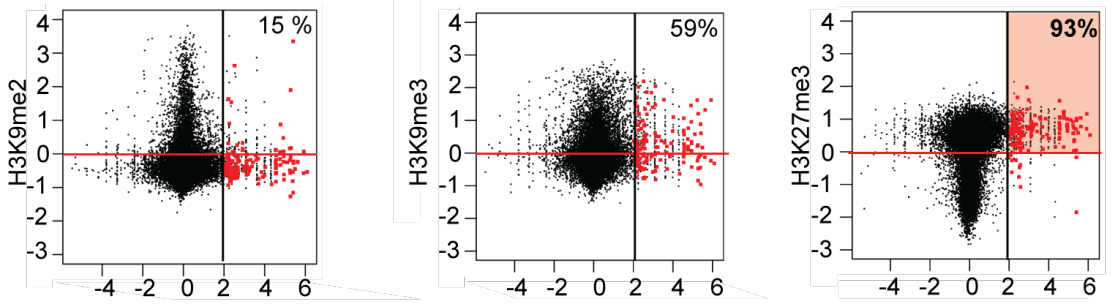

- up-regulated genes in $/ s m-8^{-/}$mutant in L1 stage (FDR $<0.05$ and Fc $>4$ )

\section{Supplementary Figure S4: Genes silenced by LSM2-8 carry the Polycomb mark}

H3K27me3, have a low steady-state expression and are not enriched on chromosome arms. 
A, Correlation of $\log 2(\mathrm{FC})$ in $l s m-8^{-/-}$with the H3K9me1 mark ChIP-seq data and with additional ChIP-seq data for H3K9me3 and H3K27me3 using different antibodies than those used in Figure 3 (see Experimental materials). B, Scatter plots comparing absolute transcript abundances $(\log 2$ of normalized reads count) of annotated genes in $l s m-8^{-/}$, met- $2^{-/-}$set- $25^{-/-}$and the triple $\left(l s m-8^{-/-}\right.$, met- $2^{-/}$set- $25^{-/}$) mutant versus WT. Boxes with pink background indicate low abundance values smaller than 6 in $\log 2$ scale for genes considered to be repressed in WT. This corresponds to 64 normalized RNA-seq reads per gene, in contrast to 1024 reads per gene represented by a value of 10. Note the large proportion among the genes upregulated in the assessed mutants (above the diagonal), which are repressed or very poorly expressed in WT. C, Distribution of upregulated genes in $l s m-8^{-/-}$along chromosomes. LEM-2 ChIP enrichment plotted over chromosomes (embryonic WT data from (Gonzalez-Sandoval et al., 2015)) is in grey, indicating proximity to the nuclear periphery. Up-regulated genes in $l s m-8^{-/}(\mathrm{FDR}<0.05$ and $\mathrm{Fc}>4)$ represented by the red dots are plotted over autosomes and X chromosome. D, Comparison between our RNA-seq and other available RNA-seq datasets (Latorre et al., 2015; Miki et al., 2016; Wang et al., 2014) in L3 stage C. elegans, for the percentage of H3K27me3-enriched genes among misregulated genes. We classify a gene as enriched for $\mathrm{H} 3 \mathrm{~K} 27 \mathrm{me} 3$, if it has positive reproducible enrichment of H3K27me3 over input from two ChIP-seq datasets from ModEncode (Table -S3). Genes upregulated in $x r n-2$ RNAi treated worms (Miki et al., 2016) but not upregulated in $l s m-8$ mutant worms are not significantly enriched for H3K27me3 (Table S2). E, Scatterplots of fold changes of L1 sorted $l s m-8$ mutant worms versus WT computed from the normalized read counts per gene in $\log 2$ scale for each of two independent RNA-seq replicate sample pairs, as in Fig. 3B. F, Scatter plots contrasting on the $\mathrm{x}$ axis the gene expression changes in L1 (Fc of sorted lsm- 8 mutant worms versus WT as determined by EdgeR) to the enrichment of the indicated histone mark over input samples (y axis), as in Fig. 3D. 


\section{Supplementary Figure 5 Mattout et al.,}

a Example of a gene upregulated in $/ s m-8^{--}$vs WT (RNA-seq L3, strand specific)

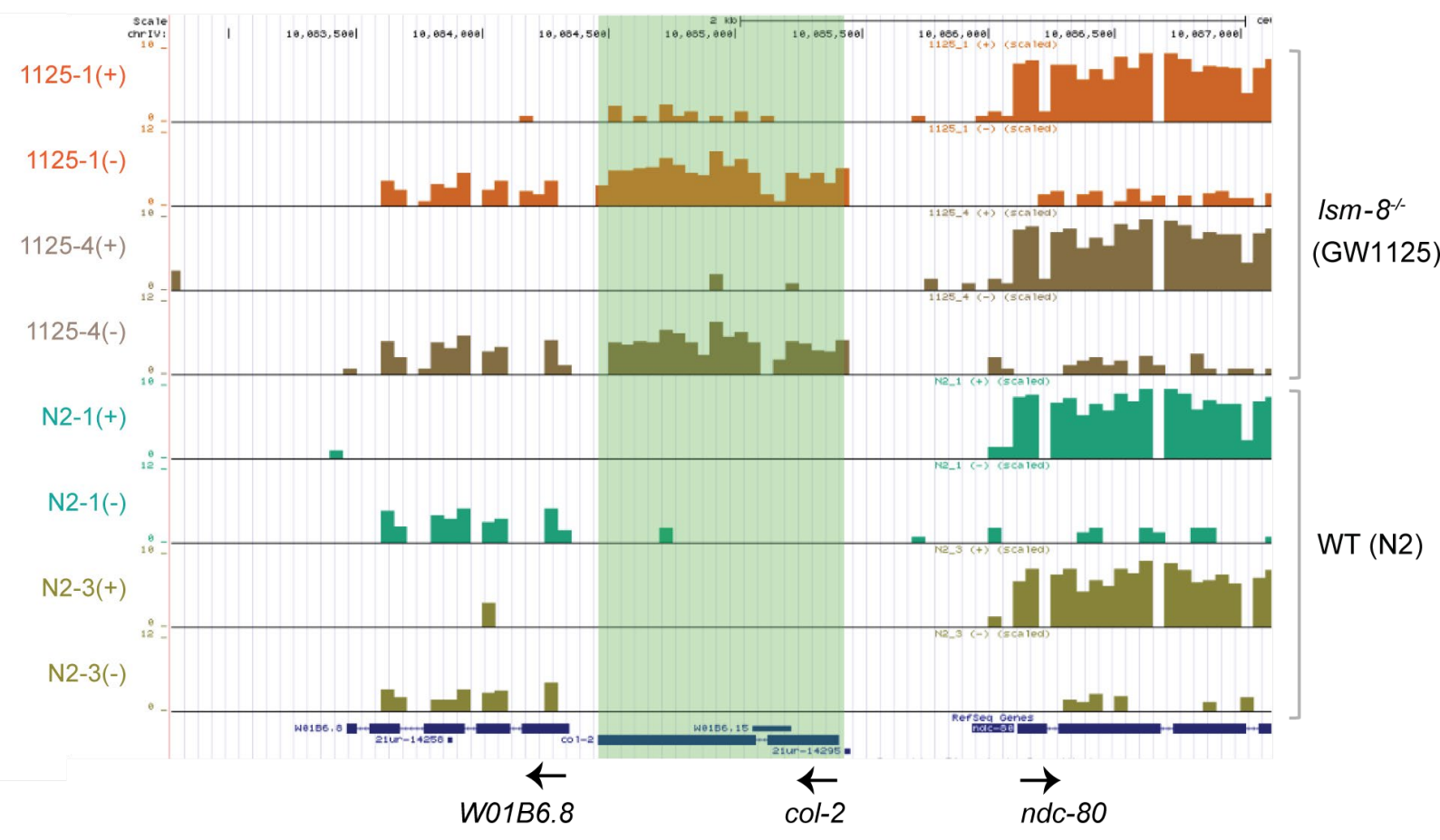

b

ChIP-seq data (ModeEncode) in WT

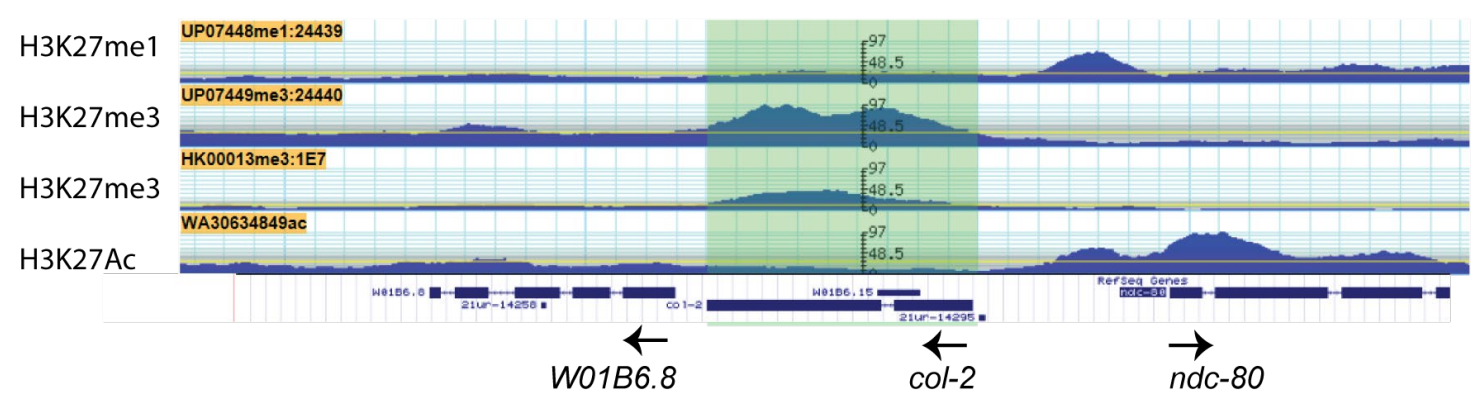

\section{Supplementary Figure S5: LSM-8 ablation does not alter transcription termination accuracy, strand specificity nor splicing.}

A, UCSC genome browser view showing wiggle tracks from positive (+) or negative (-) strands show the differential expression of the col-2 gene, which is upregulated in $l s m-8^{-/}$compared to WT (y axis in log2). The expression level of the neighboring genes is not affected and termination defects are not observed. All introns were as efficiently spliced in $l s m-8^{-/-}$as in WT. B, G browse view showing the ModEncode ChIP-seq tracks for H3K27me1, H3K27me3 (two 
different antibodies) and H3K27Ac at the same genomic locus (IV:10,082,495..10, 087, 496) around the col-2 gene, as shown in (A). The col-2 gene is upregulated in $l s m-8^{-/}$compared to WT and enriched for H3K27me3, as $95 \%$ of the genes upregulated in $l s m-8^{-/}$.

\section{Supplementary Figure 6 Mattout et al.,}
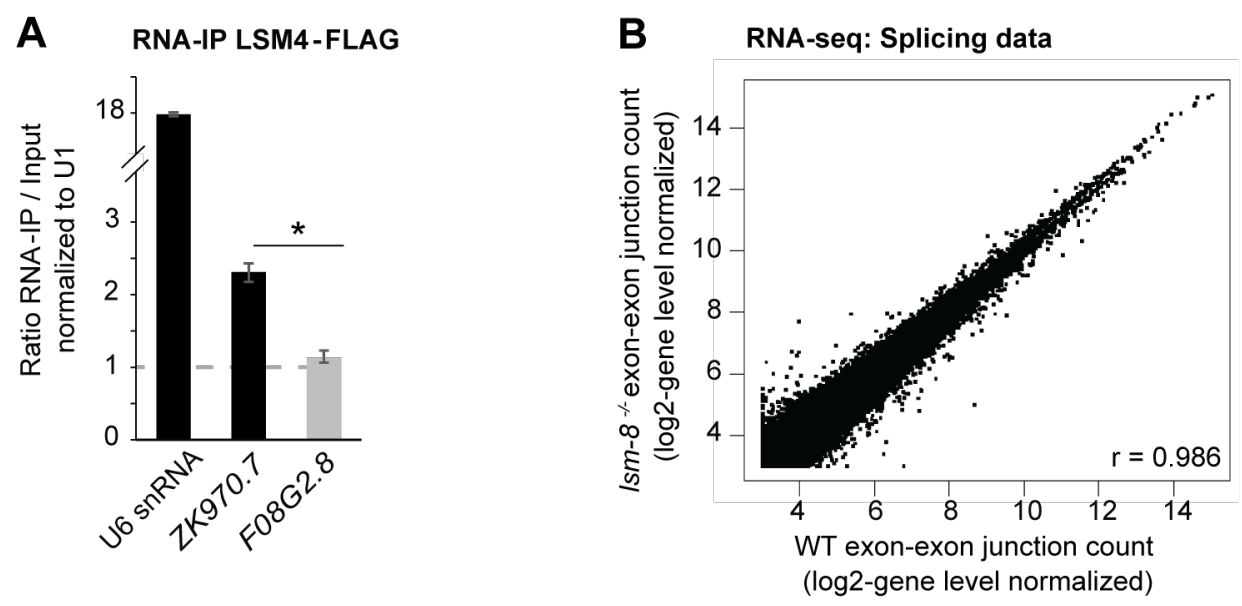

Supplementary Figure S6: $l \mathbf{s m}-8$ deletion does not affect splicing globally.

A, RNA IP-qPCR. LSM-4-FLAG RNA IP analysis in native conditions. RNA levels were normalized to input and U1snRNA levels. ZK970.7 is upregulated in $l s m-8^{-/-}\left(l_{s m}-8\right.$ target gene) and associate with LSM4 (>1), whereas F08G2.8 is not (non-target gene) and do not associate with LSM4. Those two examples suggest that the LSM-8 complex can bind to the RNAs it regulates. $\mathrm{N}=2, \mathrm{n}=3$, bars: s.e.m. $\mathbf{B}$, Reads which align on exon-exon junctions were counted in $l s m-8^{-/-}$and WT worms. Scatter plot compares exon-exon junction mapped reads $(\log 2)$ normalized to their intrinsic gene level in WT (x-axis) and $l s m-8^{-/-}$worms (y-axis). 


\section{Supplementary Figure 7 Mattout et al.,}

A

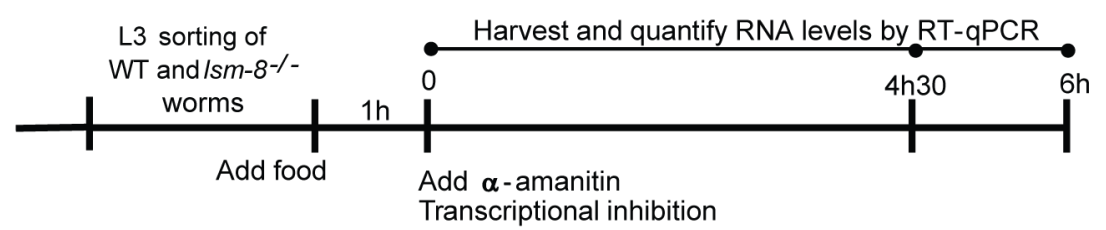

B RNA decay assay following (transcription inhibition)

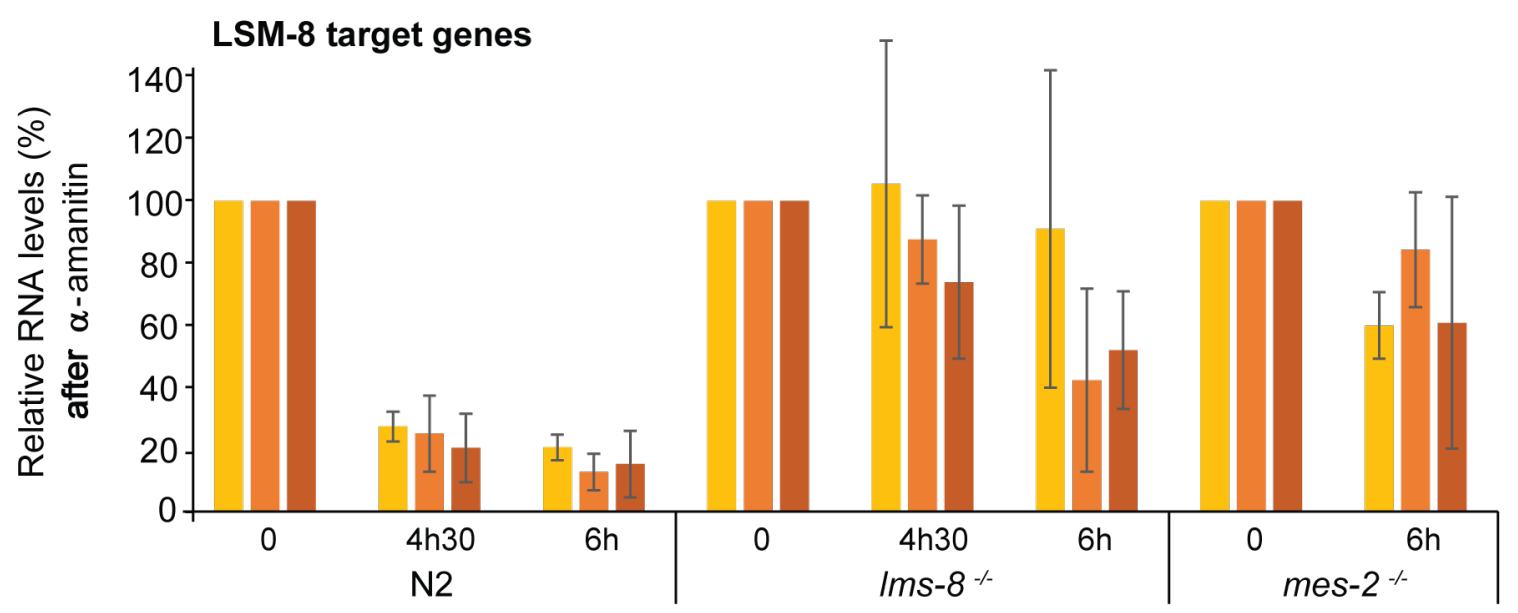

far-3 $\square$ grl-23 $=Z K 970.7$

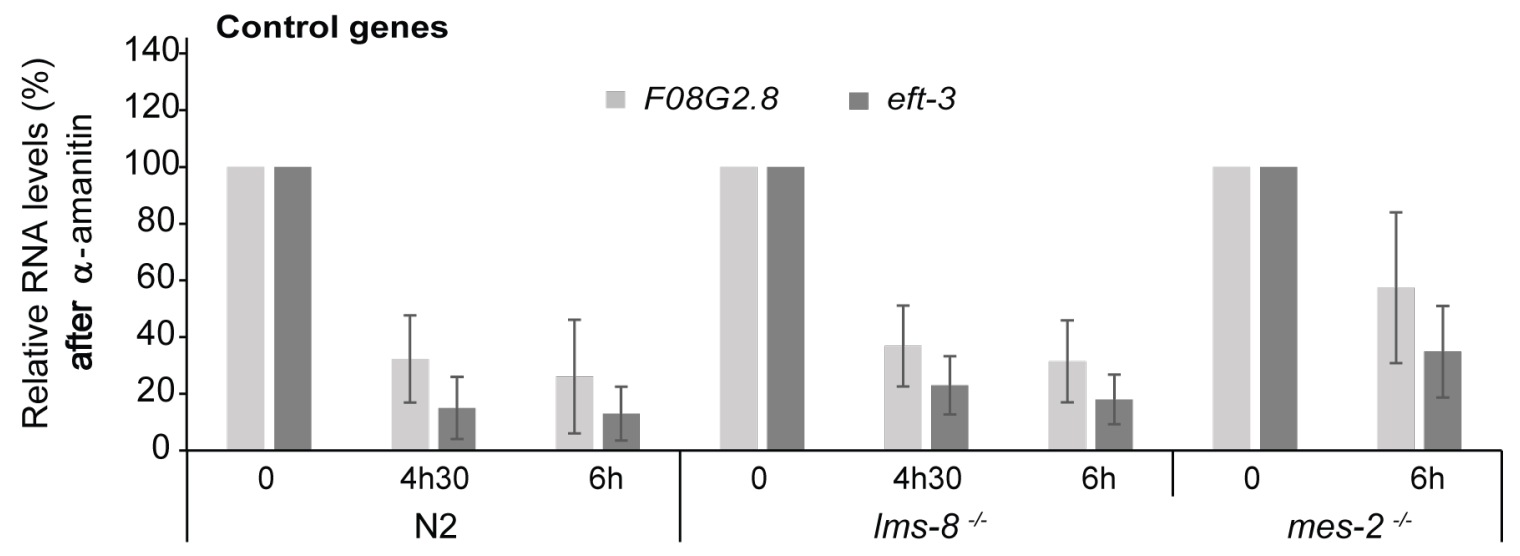

\section{Supplementary Figure S7: LSM2-8 promotes the degradation of specific transcripts.}

A, Scheme of the RNA decay assay. WT and $l s m-8^{-/}$worms were sorted, re-fed with OP50 in liquid culture for $1 \mathrm{~h}$ at room temperature and treated with $50 \mu \mathrm{g} / \mathrm{ml}$ final concentration of $\alpha$ amanitin, which inhibits Pol II and Pol III transcription. RNA was isolated at time $0,4.5 \mathrm{~h}$ and at $6 \mathrm{~h}$, as indicated for each independent experiment. B, RNA levels of three transcripts affected by LSM-8 (upper graph) and two control transcripts (expression not affected by LSM-8, lower 
graph) were determined by RT-qPCR and normalized to $18 \mathrm{~S}$ rRNA levels which are insensitive to $\alpha$-amanitin. The value at $0 \mathrm{~h}$ is defined as $100 \% . \mathrm{N}=3, \mathrm{n}=3$, bars $=$ s.e. $\mathrm{m}$.

Supplementary Table S2: RNA-seq data for genes in $l s m-8$, met-2 set-25 and $l s m-8$, met-2 set-25 triple mutant versus WT at the L3 stage. (Excel file uploaded separately)

Supplementary Table S3: Enrichment of histone marks in L3 (ModEncode Data, Excel file uploaded separately)

Supplementary Table S4: Lists of biological processes affected in the different mutants (GO analysis). (Excel file uploaded separately)

Supplementary Table S5: RNA-seq data for genes in $l s m-8$ mutant versus WT at the L1 stage. (Excel file uploaded separately)

\section{Experimental Procedures}

\section{Worm strains and growing conditions}

Table S1 lists the strains used in this study. Strains with deletion alleles and reporters obtained from the C. elegans knockout consortium or made by the CRISPR/Cas9 system were outcrossed 2 to 6 times to the N2 (WT) strain. Worms were grown on OP50 and maintained at $22.5^{\circ} \mathrm{C}$, except when frozen or manipulated at room temperature (RT).

The $l s m-8$ deletion allele xe17 (sequence below) was generated by replacing the entire coding sequence of the lsm-8 gene with the red pharynx marker [myo2p::mCherry::unc54 3'UTR] using an adapted version of the CRISPR/Cas9 technique (Katic et al., 2015). For this, the N2 worms were injected with the following mix pDD162 (Cas9, Dickinson et al., 2013) $100 \mathrm{ng} / \mu 1, \mathrm{LSM} 8$ 


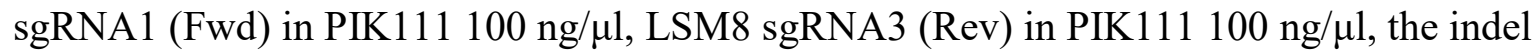
plasmid $l s m-8 \Delta$-mCherry in pIK37 $100 \mathrm{ng} / \mu \mathrm{l}$ and Pmyo-3::gfp $5 \mathrm{ng} / \mu \mathrm{l}$.

\section{DAPI staining and live microscopy}

DAPI staining was carried out on WT and $l s m-8^{-/}$(handpicked) worms from different developmental stage (not mixed) and mounted on poly-L-lysine coated slides. Two independent biological replicates were performed. The freeze cracking of worms by liquid nitrogen in Eppendorf tubes was followed by fixation for $5 \mathrm{~min}$ in methanol at $-20^{\circ} \mathrm{C}$, and $2 \mathrm{~min}$ in $1 \%$ paraformaldehyde at $\mathrm{rt}$ for all stages. After fixation, 3 x 5 min washes with PBS supplemented with $0.25 \%$ TritonX100 (PBSX) were done with the last wash optionally lasting overnight (ON) at $4^{\circ} \mathrm{C}$. DAPI $(1 \mu \mathrm{g} / \mathrm{ml})$ was added for $10 \mathrm{~min}$ at $\mathrm{rt}$ and was washed twice before mounting the slides with n-propyl gallate. For live imaging, animals were mounted on slides coated with $2 \%$ agarose pads, supplemented with $0.1 \%$ sodium azide and $1 \mathrm{mM}$ levamisole, in most cases.

Microscopy was carried out on a spinning disc confocal microscope (AxioImager M1 [Carl Zeiss] + Yokogawa CSU-22 scan head, Plan-Neofluar 100×/1.45 NA oil objective, EM-CCD camera [Cascade II; Photometrics], and VisiView 2.1.4 software, (Fig. S1D,E, Fig. 2C, Fig. 5B, Fig. S2B-E) either Axo imager 2.1 Zeiss, (Fig. 2D, Figs. 2I-L1, 3A-D). Images, 3D reconstruction (maximum intensity Z-projections) and fluorescence intensity analysis were generated using Fiji/ImageJ software.

\section{RNAi experiments}

RNAi was performed at $22.5^{\circ} \mathrm{C}$ by placing synchronized L1 worms on feeding plates as previously described (Timmons et al., 2001). Synchronized L1 larvae were obtained by bleaching gravid adults and the eggs recovered were left to hatch overnight at RT in M9. All RNAi clones used against LSM complexes subunits and used in the targeted RNAi screen were sequenced and a blast analysis performed first to confirm the specificity of the targets. At least, three independent biological replicates were performed for each RNAi experiment. As a mock RNAi control, the L4440 vector (Fire vector library) was modified by removing an EcoRV fragment containing $25 \mathrm{~b}$.

For RNAi against $x r n-2$, bacteria expressing dsRNA were diluted with mock RNAi bacteria to feed the GW306 and GW1119 strain in order to get a milder phenotype and thus enough progeny 
in which to assess derepression. Both $l s m-8$ heterozygous and homozygous worms (GW1119) were subjected to RNAi treatment, but only homozygous worms were used to assess the RNAi effect. For the RNAi with LSM-8 potential co-factors, most of the chosen candidates were LSM2-8 subunits related or controls. Co-regulated genes were predicted though a clustering analysis in SPELL (http://spell.caltech.edu:3000/). List of interacting partners were predicted through the Wormbase. The derepression was assessed by the worm sorter as described in Figure 1 for RNAi hits that produce L1 larvae in the next generation. For RNAi hits that caused larval arrest or embryonic lethality, derepression was assessed by microscopy using in each case, adequate controls.

\section{Quantitation of derepression}

Derepression was scored at specific developmental stages by fluorescence microscopy using standardized exposure and illumination conditions. Quantitation of GFP intensity in different conditions was done using Fiji/ImageJ software and the ROI manager, for semi-automated analyses. The fluorescence intensities from whole animals at similar developmental stages were also compared.

Quantitation of derepression by the worm sorter, COPAS BIOSORT (Union Biometrica), was performed in L1 worms according to manufacturer's guidelines. Visual inspection of the selected and monitored worms showed that $>99 \%$ of all worms matched the size criteria. Data corresponding to the fluorescence intensity (PH Green or PH Red) were analyzed and plotted in boxplots using R studio. The EXT (1-5) was extracted to exclude possible remaining bacteria.

\section{Survival assay}

Worms of indicated genotypes were synchronized by bleaching, and when they reached the L4 stage (Day 2 at $22.5^{\circ} \mathrm{C}$ ), ten worms were isolated onto plates containing OP50 bacteria. Four independent biological replicates were performed. The number of worms alive was determined every $24 \mathrm{~h}$. At Day 4, surviving adults worms from each genotype (even sterile ones, $l s m-8^{-/}$and met-2 set-25; $l s m-8^{-/}$) were transferred to a new plate to avoid contamination with the progeny and at Day 6, only adults of WT and met-2 set-25 strains were transferred, since the other sterile worms were too fragile to move without being killed.

\section{Chromatin Immunoprecipitation (ChIP) experiments}


$\sim 20,000 \mathrm{WT}$ and $l s m-8^{-/-}$homozygous L3-L4 larvae stage were isolated using the COPAS BIOSORT instrument (Union Biometrica), according to manufacturer's guidelines. Three independent biological replicates were performed. Visual inspection of the sorted worms showed that $>90 \%$ of all worms were expressing appropriate markers (i.e., red fluorescence but no GFP expression in the pharynx for $l s m-8^{-/}$, and no markers for the WT) and $90 \%$ matched the desired size and morphological criteria that corresponds to the stage of interest.

Antibodies used for the ChIP were rabbit anti-H3K27me3 (ChIP, Millipore, 07-449), whose specificity was confirmed by peptide binding, and IF on a mes- 2 mutant (data not shown).

H3K27me3 ChIP was performed as previously described (Zeller et al., 2016). In brief, chromatin was incubated overnight with $3 \mu \mathrm{g}$ of antibody coupled to Dynabeads Sheep Anti-Rabbit IgG (Invitrogen), in FA-buffer (50 mM HEPES/KOH pH7.5, 1 mM EDTA, 1\% Triton X-100, 0.1\% sodium deoxycholate, $150 \mathrm{mM} \mathrm{NaCl}$ ) containing 1\% SDS. Chromatin/ antibody complexes were washed with the following buffers: 3 x 5 min FA buffer; 5 min FA buffer with $1 \mathrm{M} \mathrm{NaCl} ; 10$ min FA buffer with $500 \mathrm{mM} \mathrm{NaCl} ; 5$ min with TEL buffer (0.25 M LiCl, 1\% NP-40, 1\% sodium deoxycholate, $1 \mathrm{mM}$ EDTA, $10 \mathrm{mM}$ Tris-HCl, pH 8.0) and twice for $5 \mathrm{~min}$ with TE. Complexes were eluted at $65^{\circ} \mathrm{C}$ in $100 \mu$ l of elution buffer (1\% SDS in TE with $250 \mathrm{mM} \mathrm{NaCl}$ ) for $15 \mathrm{~min}$. Both input and IP samples were incubated with $20 \mu \mathrm{g}$ of RNAse A for 30 minutes at $37^{\circ} \mathrm{C}$ and $20 \mu \mathrm{g}$ of proteinase $\mathrm{K}$ for $1 \mathrm{~h}$ at $55^{\circ} \mathrm{C}$. Crosslinks were reversed overnight at $65^{\circ} \mathrm{C}$. DNA was purified using a Zymo DNA purification column (Zymo Research).

\section{RNA-IP in native conditions}

Enriched L3 stage worms (GW1004 which contains extrachromosomal arrays expressing LSM4-GFP/3xFLAG-tagged from a fosmid which was obtained from the " $C$. elegans TransgeneOme" consortium) were collected as 300-500 $\mu \mathrm{l}$ of pelleted worms and lysed at $4{ }^{\circ} \mathrm{C}$ with a Dounce Tissue Grinder (150 strokes for each $500 \mu$, BC Scientific, Miami, FL, USA) in an equal volume of lysis buffer (30 mM HEPES/KOH pH 7.4, $100 \mathrm{mM} \mathrm{KCl,} 1.5 \mathrm{mM} \mathrm{MgCl}$, 0.1\% Triton X-100, Protease Inhibitor Cocktail Tablets, EDTA-free, Roche Rnase inhibitor, rRNAsin $1.25 \mu 1 / \mathrm{ml}$ of lysis buffer). Lysates were cleared at $16000 \mathrm{x} \mathrm{g}$ for $15 \mathrm{~min} .4 \mathrm{mg}$ of lysate proteins were incubated with $40 \mu 1$ of pre-washed anti-FLAG M2 magnetic beads (SigmaAldrich) for $2 \mathrm{~h}$. Washes were performed in lysis buffer. For RNA extraction, washed magnetic beads were resuspended with $100 \mu \mathrm{l}$ of lysis buffer and $400 \mu \mathrm{l}$ Trizol® (Ambion) and the 
samples were snap-frozen in liquid nitrogen. Two independent biological replicates were performed.

\section{RNA extraction}

For the RNA-seq experiment WT, met-2 set-25, $l s m-8^{-/-}$, and met-2 set-25; lsm- $8^{-/-}$worms were isolated using the COPAS BIOSORT instrument according to the fluorescent criteria (non-green pharynx worms) using the size criteria of L3 stage larvae in 4 independent biological replicates. For L1 RNA-seq experiment, worms were synchronized prior to the sorting process. Synchronized L1 larvae were obtained by bleaching gravid adults and the eggs recovered were left to hatch $16 \mathrm{~h}$ at RT in M9.The isolation of WT and $l s m-8^{-/-} \mathrm{L} 1$ larvae was made similarly with the fluorescent criteria (non-green pharynx worms) and the size criteria of L1 stage larvae. The larvae were refeed for $2.5 \mathrm{~h}$ after the sorting process. For all RNA based experiments, before RNA extraction, worms were washed 3x in M9 and re-suspended in $100 \mu 1$ of M9, 400 $\mu$ l of Trizol® (Ambion) and snap-frozen in liquid nitrogen.

Extraction of RNA used 4 freeze-thaw cycles from liquid nitrogen to a $42^{\circ} \mathrm{C}$ heat bath, followed by the addition of $200 \mu 1$ of Trizol ${ }^{\circledR}$ to each sample. Vigorous vortexing at room temperature (rt) in 5 cycles ( $30 \mathrm{sec}$ vortex, $30 \mathrm{sec}$ on ice), was followed by 5 min at rt. RNA extraction was with $140 \mu 1$ chloroform, vigorous shaking for $15 \mathrm{sec}$, and $2 \mathrm{~min}$ at $\mathrm{rt}$. The samples were centrifuged at $12000 \mathrm{rcf}$ at $4^{\circ} \mathrm{C}$, and the aqueous phases were transferred to fresh tubes. An equal volume of $70 \%$ EtOH was added slowly and the homogeneous mixture was transferred to a Qiagen RNeasy spin column (RNeasy kit, QIAGEN 74104). QIAGEN protocols including a subsequent $30 \mathrm{~min}$ DNAse treatment. For L1 RNA-seq samples, the extraction was done using the Zymo DirectZol microRNA kit (R2060).

\section{RT-qPCR}

Primers were designed to be exon-junction spanning where possible, and are listed below. cDNA synthesis was performed using the (AMV cDNA kit, NEB, E6550S) according to the manufacturer's protocol using random primers and 0.1-3 $\mu \mathrm{g}$ of total RNA per sample according to the experiment. qPCR was performed on a StepOnePlus real time PCR system (Applied Biosystems) using SYBR Green Mastermix (Applied Biosystems; 4309155). Further analysis was done in Microsoft Excel. All primer pairs were tested and selected for amplification efficiencies ranging from $85-100 \%$. For gene expression analysis in Fig. 1 and S1, $\Delta \Delta$ CT method 
was used, his-56 and pmp-3 were used for sample normalization. For ChIP-qPCR, sample data were normalized to corresponding input chromatin. Candidate genes were chosen in Fig. 6 based on their expression changes and on their enrichment for H3K27me3 in WT worms. For RIPqPCR in Fig. S6A, RNA levels were normalized to corresponding input and to the U1snRNA levels.

\section{RNA decay assay}

WT, $l s m-8^{-/-}$and $m e s-2^{-/-}$(F2) L3 larvae were sorted and re-fed with OP50 in liquid culture for 1 $\mathrm{h}$ at RT. Subsequently $\alpha$-amanitin (Sigma-Aldrich) was added to a final concentration of 50 $\mathrm{mg} / \mathrm{ml}$, to block transcription and stall larval development (Miki et al., 2014). About 750 worms were harvested in duplicate in each of the three independent biological replicates, and for each sampling point. They were washed twice with M9 medium, resuspended in $400 \mathrm{ml}$ of Trizol ${ }^{\circledR}$ (Life Technologies) and frozen in liquid nitrogen. To assess the RNA decay, RNA levels of genes affected or not by the LSM2-8 complex (expression level) were quantified before and after the transcriptional inhibition in each genotype. LSM-8 target genes were selected by their higher expression levels in $l s m-8^{-/-}$versus WT (RNA-seq), and their enrichment for H3K27me3 in L3 larvae, yet it was desired to have detectable levels in WT control. In this assay, cDNA was generated from total RNA by the SuperScript III First-Strand Synthesis System (Thermo Fisher Scientific) using random primers and the 5x FS buffer for better yields. Three micrograms of total RNA were used as a template for reverse transcription reaction $(20 \mu \mathrm{l})$, and $0.66 \mu 1$ of the reaction was used for qPCR reaction $(10 \mu \mathrm{l})$. RT-qPCR for this assay was performed using PowerUp SYBR Green Master Mix (Thermo Fisher Scientific), specific primers for mature/spliced mRNAs (complementary to an exon-exon junction; grl-23, F08G2.8) or for preand mature mRNAs (far-3, ZK970.7) or for pre-mRNA only (eft-3) and using StepOnePlus Realtime PCR Systems (Applied Biosystems) according to the suppliers' protocols. For primer sequences for eft-3 and 18S ribosomal RNA, see (Miki et al., 2014). Because pre-mRNA levels are expected to be more directly affected by transcription inhibition, eft-3 pre-mRNA was used by us and by others (Miki et al., 2014) as a control for the efficiency of the $\alpha$-amanitin treatment in inhibiting transcription. The high expression levels of eft-3 makes it an adequate control to verify the potential extent of the transcriptional inhibition. In addition, eft-3 is also a control gene in the sense that it is not regulated by $l s m-8$. 


\section{RNA-seq}

Total RNA was treated for the L3 samples additionally with the Turbo DNA free kit (Ambion, AM1907), depleted for rRNA using Ribo-Zero Gold kit from Epicentre and depletion validated through Agilent Bioanalyzer analysis. Subsequent library preparation was performed with a ScriptSeq v2 RNA-Seq library preparation kit, stranded (Epicentre). Library preparation for the L1 samples was performed with the TrueSeq Total RNA preparation kit, stranded (Illumina).

The quality of the resulting libraries was assessed with an Agilent Bioanalyzer and concentrations were measured with a Qubit fluorometer prior to pooling. $50 \mathrm{bp}$ single-end sequencing was done on an Illumina HiSeq 2500.

\section{Processing of the RNA-seq and ChIP-Seq data}

The RNA-seq samples from four independent biological replicate samples L3 were mapped to the C. elegans genome (ce6) with the R package QuasR v1.22.0, (www.bioconductor.org/packages/2.12/bioc/html/QuasR.html) with the included aligner bowtie (Langmead et al., 2009) considering only uniquely mapping reads for mRNA. The command "proj <-qAlign("samples.txt","BSgenome. Celegans.UCSC.ce6")" instructs bowtie to align using the parameters "-m 1 --best --strata --phred33-quals". Since the used replicas differed slightly in timing (Fig. S3A), we incorporated a blocking factor in the linear model treating the replicates as different batches. For splice junction quantification we used the spliced alignment algorithm SpliceMap (Au et al., 2010). The command used was "proj <qAlign("samples.txt","BSgenome.Celegans. UCSC.ce6",splicedAlignment=TRUE)". The command to create various count tables was qCount(proj,exons, orientation="same"). For gene quantification, gene annotation from WormBase was used (WS190). The EdgeR package v 3.24.0 was used to determine fold changes $(\mathrm{Fc})$ and false discovery rates (FDR) of differential transcript abundances. The repeat element quantitation was based on UCSC (genome.uscsc.edu) repeat annotation. To normalize for sequencing depth, each sample was divided by the total number of reads and multiplied by the average library size. Transformation into log2 space was performed after the addition of a pseudocount of 8 in order to minimize large changes in expression caused by low count numbers. The various count tables used throughout this study were normalized separately. To determine the developmental timing of each RNA-seq sample, we previously used a set of 2050 genes shown to gradually rise between $25 \mathrm{~h}$ and $36 \mathrm{~h}$ post 
hatching at $25^{\circ} \mathrm{C}$ (all rising genes)(Hendriks et al., 2014). While most of those genes are germline genes and thus stop being expressed in $g l p-4$ mutants which are devoid of germ cells (Hendricks et al., 2014, Fig. S3A), we noticed that a subset of those rising genes ( $\mathrm{n}=162)$ actually still continued to rise even in glp-4 mutant worms (Hendricks et al., 2014, Fig S3A). We therefore split the 2050 genes into two separate groups, a germline developmental signature $(n=1888)$ and a somatic developmental signature $(\mathrm{n}=162)$ and used the latter to infer developmental timing (Fig S3A). We got the same result using all rising genes (data not shown). To quantify potential changes in splicing in $l s m-8^{-/-}$as opposed to WT, we quantified the expression of all the exonexon junctions from the spliced alignments using no annotation. The command used to create the exon-exon junction count table was qCount(proj2,NULL,reportLevel="junction"). These junction counts were then normalized for library size (as described above) and overlapped with gene annotation to assign them to their host gene. Junctions overlapping multiple genes were discarded. The assignment to the host gene was then used to correct the junction expression levels for differences in gene expression. This was done by dividing the junction counts of either WT or $l s m-8^{-/}$by the respective gene expression change depending on the direction of the change. This procedure ensured that junction counts were always deflated and not inflated by the gene expression correction. Finally a pseudocount of 8 was added and the data were $\log 2$ transformed. We specifically chose to not use reads overlapping intronic sequences for this analysis as they can reflect changes in mRNA transcription (Habacher et al., 2016) and thus would potentially complicate the interpretation of those results in the light of alternative splicing. The RNA-seq L1 samples were mapped to the C. elegans genome (ce10) and processed otherwise as mention above (no blocking factor applied, as for L3). The ChIP-seq data for L3_H3K9me1/2/3 (5036, 5050, 5037, 5040), L3_H3K27me3 $(5045,5051)$, L3_H3K27ac (5054), L3_H3K4me2/3 $(5055,3576)$ were downloaded from ModEncode (http://data.modencode.org/) and mapped to ce6 and ce10 using bowtie considering only uniquely mapping reads. Quantitation for each gene was performed by counting the reads overlapping the gene-body. All samples were normalized for total library size, $\log 2$ transformed after adding a pseudocount of 8 and and Fc enrichments $(\log 2)$ were calculated by subtracting the $\log 2$ transformed values of the specified input sample (3576, Rep-1) from each ChIP-seq sample. 
Misregulated genes in the prg-1 and lin-35 mutants (Latorre et al., 2015; Wang et al., 2014) were converted into WB gene names through the Gene ID conversion tool (DAVID), and the resulting genes were compared to their enrichment in $\mathrm{H} 3 \mathrm{~K} 27 \mathrm{me} 3$ similarly as for the misregulated genes in the $l s m-8$ mutant (Table S3).

\section{Primers used in this study}

\section{Primers Other Name Sequence}

\begin{tabular}{|c|c|c|}
\hline \multicolumn{3}{|l|}{ Cloning } \\
\hline FA115 & Gibson_pIK37_lsm-8up_fwd & acacaacatatccagtcactatg \\
\hline FA116 & Gibson_Pmyo2_lsm-8up_rev & ctactcagatataaaatgcaact \\
\hline FA117 & Gibson_lsm-8up_Pmyo2_fwd & atctcacttgggaacaataa \\
\hline FA118 & Gibson_lsm-8down_GFP_rev & accagtcaatacaacgcgtc \\
\hline FA119 & Gibson_GFP_lsm-8down_fwd & accaaacataactgtttccaaca \\
\hline FA120 & $\begin{array}{l}\text { Gibson_pIK } 37 \text { _lsm- } \\
\text { 8_down_rev }\end{array}$ & ggcgtgtcaataatatcact \\
\hline sgRNA1 & LSM8 sgRNA 1 & aattgcaaatctaaatgtttage \\
\hline sgRNA3 & LSM8 sgRNA 3 & aattgcaaatctaaatgtttaa \\
\hline SG6643 & Fwd Lsm-8 NotI primer & attagcggecgecttggcge \\
\hline SG6644 & Rev Lsm-8 XhoI primer & aaatctcgagccaaattggt \\
\hline \multicolumn{3}{|c|}{ Genotyping and sequencing } \\
\hline SG7048 & 1sm-5 fwd primer for genotyping & gctttaaaattcaaaattcc \\
\hline SG7049 & 1sm-5 rev primer for genotyping & getggaataatcgaaaatc \\
\hline SG7050 & 1sm-5 rev2 primer for genotyping & ccatgttcacgtagtcatcg \\
\hline SG7051 & gut-2 fwd primer for genotyping & ggtggagtgtaatcgggatg \\
\hline SG7052 & gut-2 rev primer for genotyping & cgactaaacaacagtcgacc \\
\hline SG7053 & gut-2 rev2 primer for genotyping & cccaggaatggcacttgcg \\
\hline SG7370 & lsm4 ok3151 deletion fwd & tcagttgccactttctcttct \\
\hline SG7371 & lsm4 ok3151 deletion rev & aaacaacccgacttggggaa \\
\hline SG7175 & lsm8 deletion Cas9 fwd & cacccgtaaattcgctccca \\
\hline SG7176 & 1sm8 deletion Cas9 rev & cgttgaaatcgagcactggaa \\
\hline SG7177 & lsm8 deletion Cas9 rev2 & tgactggcgaaggtatgtcg \\
\hline SG7381 & lsm8 Fwd for genotyping & tgaatgcgaacaggtgggtt \\
\hline SG7382 & lsm8 Rev for genotyping & gggagaagaaatggtggggg \\
\hline SG7413 & lsm8 Rev for genotyping & ccaaattggtgccagtgac \\
\hline SG7445 & lsm8 genotyping/sequencing & aggtgtccegtcttcgtcta \\
\hline SG7446 & lsm8 genotyping/sequencing & gggagaagaaatggtggggg \\
\hline SG7447 & lsm8 genotyping/sequencing & atgaatcgtcaggtgtcceg \\
\hline SG7449 & lsm8 genotyping/sequencing & aggtgtccegtcttcgtcta \\
\hline SG7450 & lsm8 genotyping/sequencing & gggagaagaaatggtggggg \\
\hline SG7451 & lsm8 genotyping/sequencing & atgaatcgtcaggtgtcccg \\
\hline SG7452 & lsm8 genotyping/sequencing & ggggagaagaaatggtgggg \\
\hline SG7453 & lsm8 genotyping/sequencing & ttgaatgcgaacaggtgggt \\
\hline
\end{tabular}




$\begin{array}{lll}\text { SG7576 } & \text { 1sm8 genotyping/sequencing } & \text { gctggattttgaagacggcg } \\ \text { SG7666 } & \text { 1sm8 genotyping/sequencing } & \text { tgctttttggggtttccect } \\ \text { SG7667 } & \text { 1sm8 genotyping/sequencing } & \text { gctggatttgaagacggcg } \\ \text { SG7668 } & \text { 1sm8 genotyping/sequencing } & \text { tgccaggagcgtacaatgtt } \\ \text { SG7669 } & \text { 1sm8 genotyping/sequencing } & \text { ctaccaccacgaccgctaaa } \\ \text { SG6798 } & \text { sequencing insertion into PIK37 } & \text { cgtctcgagtgtaaaacgacg } \\ \text { SG9482 } & \text { dcap2 Fwd for genotyping } & \text { agtacgacgtggtccatttcc } \\ \text { SG9583 } & \text { dcap2 Rec for genotyping } & \text { attttgcattttcgtcatcacat } \\ \text { SG9484 } & \text { 1sm1 Fwd for genotyping } & \text { atcatggacttgccegatcc } \\ \text { SG9485 } & \text { lsm1 Rev for genotyping } & \text { cctccagccgacgaaattct }\end{array}$

\section{RT-qPCR}

SG7059

SG7060

SG5830

SG5831

SG6979

SG6980

SG6981

SG6982

SG6983

SG6984

SG7087

SG6987

SG6988

SG7921

SG7923

SG9505

SG9503

SG9504 his-56 fwd for qPCR

his-56 rev for qPCR

pmp-3 fwd for qPCR

pmp-3 rev for qPCR

1 sm-8 Fwd for qPCR

1sm-8 Rev for qPCR

1sm-1 Fwd for qPCR

$1 s m-1 \mathrm{Rev}$ for qPCR

1sm-7 Fwd for qPCR

1sm-7 Rev for qPCR

1sm-7 Rev for qPCR

gfp Fwd for qPCR

gfp Rev for qPCR

gfp Fwd2 for qPCR

mRNA3 Fwd for qPCR

mRNA3 Rev for qPCR

Pre-mRNA4 Fwd for qPCR

Pre-mRNA4 Rev for qPCR

\section{RNA decay assay and RNA-IP}

SG9078

SG9079

SG9080

SG9081

SG9082

SG9083

SG9329

SG9330

SG9090

SG9091

SG9334

SG9335

SG9338

SG9339

SG9348

SG9349

SG7797

SG7798 grl-23 mature mRNA Fwd Primer

grl-23 mature mRNA Rev Primer

Y37H2A.14 mature mRNA Fwd

Y37H2A.14 mature mRNA Rev

F08G2.8 mature mRNA Fwd

F08G2.8 mature mRNA Rev

pre-mRNA eft-3 Fwd Primer

pre-mRNA eft-3 Rev Primer

18S rRNA Fwd Primer

18S rRNA Rev Primer

ZK970.7 pre\&mRNA Fwd

ZK970.7 pre\&mRNA Rev

far-3 pre\&mRNA Fwd

far-3 pre\&mRNA Rev

pre-mRNA lmn-1 Fwd Primer

pre-mRNA lmn-1 Rev Primer

U1 snRNA Fwd

U1 snRNA Rev ggtttcctcgtagatcaatcc

aaagttctccgtgataacatcc

gttccegtgttcatcactcat

acaccgtcgagaagctgtaga

ttctgagacggaaggtgtgc

ccaaattggtgcaagtgggg

geccgatccetatttacceg

cctccagccgacgaaattct

gcgtcaagttccaaggagga

ttccgcgagccacaataaga

ttgctcgagtccatctgctg

ggagagggtgaaggtgatgc

cataaccttcgggcatggca

gttccatggccaacacttg

ctacaagacacgtgctgaagtc

tccattctttgtttgtctgcca

aaattttcagccaacacttgtc

cgtgtacataaccttcgggc

tctcaacgagaacaccaagga

aagtgagcagaagtcgtccg

aggttcgagtgaaggaagtga

gcgattcatcgtgggctttc

gttgaaatcgcgaatcgaccg

atcgaactggggccatttgt

acttgatctacaagtgcggagga

gtcgetggccaagactacat

cagaccaaacgttttcggacgttg

ttggacgtggtagccgtttctaag

tccgttgcaatggtgttctgt

tctttgctcccatagcggtc

tgcatacaaggcattgccag

aaaacttggcgactcctccg

ggaaactgatgcggtggtct

cgagctcatcttgagccgaa

acttacctggetggggtta

gaccaaaagtgcaatgggt 


$\begin{array}{lll}\text { SG7793 } & \text { U6 snRNA Fwd } & \text { agagaagattagcatggcccc } \\ \text { SG7794 } & \text { U6 snRNA Rev } & \\ \text { ggaacgcttcagaatttgc } \\ \text { H3K27me3 ChIP-qPCR } & \\ \text { SG8665 } & \text { f_1 qPCR primer for grl-23 } & \text { aagtgagcagaagtcgtccg } \\ \text { SG8666 } & \text { r_1 qPCR primer for grl-23 } & \text { gtcgctggecaagactacat } \\ \text { SG8651 } & \text { f_1 qPCR primer for col-2 } & \text { tttgcttgaacaccctcaga } \\ \text { SG8652 } & \text { r_1 qPCR primer for col-2 } & \text { ttagaagcgtgccgtttgtg } \\ \text { SG8643 } & \text { f_1 qPCR for Y69A2AR.12 } & \text { caacgttgccctcgacaaag } \\ \text { SG8644 } & \text { r_1 qPCR for Y69A2AR.12 } & \text { tggtgtgtttttcgttgcct } \\ \text { SG8657 } & \text { f_1 qPCR primer for col-36 } & \text { gtccagcagctccgtttact } \\ \text { SG8658 } & \text { r_1 qPCR primer for col-36 } & \text { agctctgcacttccaccatc } \\ \text { SG8669 } & \text { f_1 qPCR primer for C13A2.12 } & \text { tccgatgagcctgaacactc } \\ \text { SG8670 } & \text { r_1 qPCR primer for C13A2.12 } & \text { ttcccttttaccatgccet } \\ \text { SG8483 } & \text { duox2 1_f for qPCR } & \text { tcatccgagatctcacaagtcc } \\ \text { SG8484 } & \text { duox2 1_r for qPCR } & \text { aatcgagcgtttctatgtcgc } \\ \text { SG8503 } & \text { ska1_promoter 1_f for qPCR } & \text { acggaacacattctgcgtca } \\ \text { SG8504 } & \text { ska1_promoter 1_f for qPCR } & \text { aaatgggcaggacgtgagtt }\end{array}$

\section{xe17 allele [lsm8 indel myo2p::mcherry::unc-54 3'UTR] and flanking regions}

\footnotetext{
1 tacgaccgga ctaacggccg ctccagaggg ttcgcatttg tcgagttcac cactggagag 61 ggctgtaaat tggcccttgc tgctcgcgaa caaaccatca agggaaatc tgtgagttct 
1861 gaaattttg aggactagtt gttcttatat tatcattaaa ttttatgct tcatgaattg 1921 aatgcgaaca ggtgggttgc cgtgaaaat atctcacttg ggaacaataa acAGTTGCAT 1981 TTTATATCTG AGTAGTATCC TTTGCTTTAA ATGTCCATAA AACTAATTTT ATAATCAATA 2041 AAACAACGTT TGTAAATCAA CTGAGTTTAC AAgTAGAGAC ATTGAgGgAT ACTTTCACTA 2101 TGCTAAAGTG ATAATCGACC AAATAATAAC TTCACTTTGg TATTTATTCC TGTCTTATAA 2161 AtgTtATGTA TGAATtAAAT TCATATGCAT ATGGCTCACT CTGACAATTT TTAATAATCT 2221 TCCAGATCAA TATTGACTAC CGATGCGGGT GGTCTTTTGC TTTGAATTCT GCTGAACTTT 2281 ACACCCCGAA CAgCAATGTg tgCtTCAGCC TAAAAAAAAg TAAgTGTGTT AATCAGTGCC 2341 CCCGATTCTT CATTTTTTGC CCCTCTCTCC CGTTTCGTCG GCAAAAGAAg AgAAAATAAA 2401 gAtAAgtCTC AAgAtAggtT ggtAAtCGCt AAAgtggttg tgtggatAAg AgtAgcAAAA 2461 TGGCAGGAAG AGCACTTTGC GCGCACACAC TGTACTCATT GTTCTGGATA AAATTCTCTC 2521 GTTGTTTGCC GTCGGATGTC TGCCTCTCTG CATTGAGCCG GCTTCTTCAC TATCTTTAGT 2581 TAACCTAAAA TGCCGTTTCT TTTCTCGTAT CCCCACTATC CCGTTGAGGT TCTCTGCTCT 2641 CTTCGCTCCC TACCGCCAGC GAGCAACTAT CCGTGGGGGC GCCTTGCTCG GAAGATGGGG 2701 GgGAAGAAAg AAGATTTTTG CTATTTGCAC TTGAGAAAGA GACTTTTCCT GCGTCGATGG 2761 TtAgAgAACA GTGTGCAgAC ACTTTTCAgC TACCTAGAAT tACAATtgGA TATCCCCGCC 2821 TCCCAATCCA CCCACCCAGG GAAAAAGAAG GGCTCGCCGA AAATCAAAGT TATCTCCAGG 2881 CTCGCGCATC CCACCGAGCG GTTGACTTCT CTCCACCACT TTTCATTTTA ACCCTCGATC 2941 GTCAGACACA GAAATGACAA GTTTGTACAA AAAAGCAGGC TTAATGGTCT CAAAGGGTGA

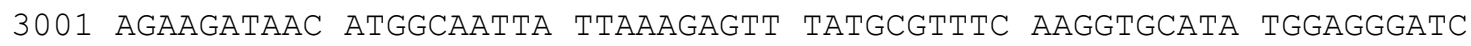

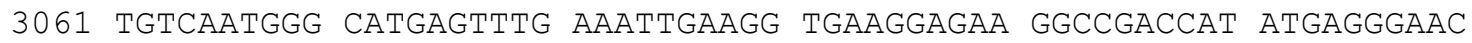
3121 ACAAACCGCA AAACTAAAGg TAAGTTTAAA CATATATATA CTAACTAACC CTGATTATTT 3181 AAATTTTCAG GTAACTAAAg GCGGACCATT ACCATTCGCC TGGGACATCC TCTCTCCACA 3241 GTTCATGTAT GgAAgtAAAg CTTATGTTAA ACATCCGgCA GATATACCAg ATTATTTGAA 3301 ACTTTCATTC CCGgAgggtT TTAAgTGgGA ACGCGTAATG AATTTTGAAg ACGgAgGAGT 3361 TGTTACAGTG ACGCAAGACT CAAGGTAAGT TTAAACAGTT CGgTACTAAC TAACCATACA 3421 TATTTAAATT TTCAGCCTCC AAgATGGAGA ATTTATTTAT AAAGTCAAAC TTCGAGGAAC

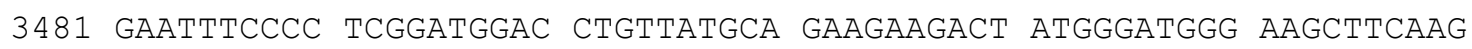
3541 TGAAAGAATG TACCCTGAAG ACGgTGCTCT TAAgGgAgAg ATTAAACAAC GTCTTAAATT 3601 GAAAGATGGA GGACATTACG ATGCTGAgGt AAgTTTAAAC ATGATTTTAC TAACTAACTA 3661 ATCTGATTTA AATTTTCAgG TGAAgACAAC TTACAAAGCC AAAAAACCAG TTCAGCTGCC 3721 AgGAGCGTAC AATGTTAATA TTAAACTGGA TATCACCTCC CACAACGAGg ATTACACTAT 3781 CGTTGAGCAA TATGAAAGA CTGAAGgGCG GCACTCGACA GGTGGCATGg ATGAATtGTA 3841 TAAGTAGTAC CCAGCTTTCT TGTACAAAGT GGGTGATATC TGAGCTCCGC ATCGGCCGCT 3901 GTCATCAGAT CGCCATCTCG CGCCCGTGCC TCTGACTTCT AAGTCCAATT ACTCTTCAAC 3961 ATCCCTACAT GCTCTTTCTC CCTGTGCTCC CACCCCCTAT TTTTGTTATT ATCAAAAAAC 4021 TTCTCTTAAT TTCTTTGTTT TTTAGCTTCT TTTAAGTCAC CTCTAACAAT GAAATTGTGT 4081 AgATTCAAAA ATAGAATTAA TTCGTAATAA AAAGTCGAAA AAAATTGTGC TCCCTCCCCC 4141 CATTAATAAT AATTCTATCC CAAAATCTAC ACAATGTTCT GTGTACACTT CTTATGTTTT 4201 TTACTTCTGA TAAATTTTT TGAAACATCA TAGAAAAAAC CGCACACAAA ATACCTTATC 4261 ATATGTTACG TTTCAGTTTA TGACCGCAAT TTTTATTTCT TCGCACGTCT GGGCCTCTCA 4321 TGACGTCAAA TCATGCTCAT CGTGAAAAAG TTTTGGAGTA TTTTTGGAAT TTTTCAATCA 4381 AgTGAAAGTT TATGAAATTA ATTTTCCTGC TTTTGCTTTT TGGGGTTTCC CCTATTGTTT 4441 GTCAAGATTT CGAGGACGgC GTTTTTCTTG CTAAAATCAC AAGTATTGAT GAGCACGATG 4501 CAAGAAAgA CGgAAgAagg TTtgggtTtg AggCtCAgtg GAAggtgagt AgAAgtTgAT 4561 AATTTGAAAg TGgAgtAgtg tCTATGgGgt TTtTgCCTTA AATgACAgAA TACATTCCCA 4621 ATATACCAAA CATAACTGTT TCCAACattg acgcgttgta ttgactggtt attctcatcg 4681 tttcccccca ccatttcttc tccccattta aatcatttt cccaactat tctccattct 4741 tttcttgtt ccttgacggt tttgtattca taccatcgtt tctttaccat ttttggatct 4801 ctaatttcct ctcaaatatt gtgaaaattt atacatattt atttgctttg tttttaatag 4861 ttaatgtatt aaatatgcat ttattcaaat tgcaacgtca ttgtatttt tcgtgtttca 4921 accgtttgtt tatcggttta acatttctt ttttaatatc gtttcctcgt tgaactttgc 4981 cattccacag aaataacctt gcagaaata ttccagtgct cgatttcaac ggaatatacg 5041 gatcgaagtt gcaaaatgag cagtatcaga gtgagttaaa tagaatgggg aaaggataat 5101 tttgaacaag agtttttgcg cgttttcaa ctttcctacc agaataaag cataagtaa 5161 aatcgataa acattttttc cagaaccaga acgacaatcg tcgaccaaca ttccgtgatc 5221 atcgtacacc acaatttagc ggtcgtggtg gtagtggtgg tggcggacgc cgtcttcaaa 
bioRxiv preprint doi: https://doi.org/10.1101/701581; this version posted July 14,2019 . The copyright holder for this preprint (which was not certified by peer review) is the author/funder, who has granted bioRxiv a license to display the preprint in perpetuity. It is made available under aCC-BY-NC-ND 4.0 International license.

5281 atccagctca ttatccacaa cgcagagata tgtcgccgat tcgcagatct tcagcaatt 5341 cacca 